\title{
Tunable Twisting Motion of Organic Linkers via Concentration and Hydrogen-bond Formation
}

\author{
Thesis by \\ Abdullah Alturki \\ In Partial Fulfillment of the Requirements \\ For the Degree of \\ Master of Science
}

King Abdullah University of Science and Technology

Thuwal, Kingdom of Saudi Arabia

January, 2019 


\section{EXAMINATION COMMITTEE PAGE}

The thesis of Abdullah Alturki is approved by the examination committee

Committee Chairperson: Omar M. Mohammed

Committee Members: Kuo-Wei Huang, Osman M. Bakr 
(C) January, 2019

Abdullah Alturki

All Rights Reserved 


\title{
ABSTRACT \\ Tunable Twisting Motion of Organic Linkers via Concentration and Hydrogen-bond Formation
}

\begin{abstract}
Abdullah Alturki
Benzothiazole dibenzoic acid derivative (BTDB) is well-known organic linkers utilized for the syntheses of various metal organic frameworks, and demonstrates interesting photophysical properties upon concentration variations in solution. The presence of two carboxylic acid functional groups at each side of the rod-like molecule, facilitates dimerization and oligomerization equilibria. Interestingly, dimers and oligomers have completely different emission behaviors from the monomer of the same species. At a low range of concentration, $0.1-64 \mu \mathrm{M}$, dimerization process is dominant, and that the equilibrium constant of dimer formation found to be $18,000 \mathrm{M}^{-1}$. On the other hand, in the $64-1000 \mu \mathrm{M}$ concentration range, oligomerization takes over, and that it results in the formation of a small linear chain of 8 molecules, or 4 dimers, with a high equilibrium constant of $1.2 \times 10^{13} \mathrm{M}^{-3}$. Various experimental measurements and theoretical calculations have suggested hydrogen-bond formation is the main driving force for the dimerization and oligomerization in the nano- and micro- molar regime, and that structure rigidity of a species is a key factor in controlling its photophysical properties, such as emission quantum yield and excited state lifetime.
\end{abstract}




\section{ACKNOWLEDGEMENTS}

I would like to express my thanks and gratitude to my academic advisor and chair of my thesis defense committee, Professor Omar F. Mohammed, whose support and dedication to my growth and development was an integral part of my academic experience. I also extend my appreciation to Professor Kuo-Wei Huang, and Professor Osman M. Bakr for their participation in the thesis defense committee.

Thanks are extended to Dr. Ahmed El-Zohry for his contribution to the success of the research project, along with his dedication to my learning progress during the course of time at KAUST. Furthermore, I would like to acknowledge the contribution of Professor Mohamed Eddaoudi to the completion of the project. Contribution acknowledgments are

also extended to Jun Yin, Arijit Mallick, Osama Shekhah, Lutfan Sinatra, Abdel Hamid Amwas, Jafar Khan, and Abdulhadi Alhaji.

Thanks are extended to my parents for their continuous support throughout my academic career. I am extremely grateful for all their efforts, and in helping shape the person I have become today. 


\section{TABLE OF CONTENTS}

EXAMINATION COMMITTEE PAGE

1.1 Organic Linkers in MOFs..................................................................... 17

1.2 Excited-State Dynamics of Organic Molecules............................................... 20

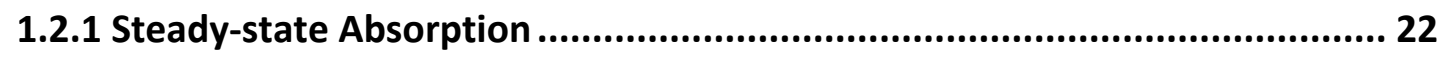

1.2.2 Non-radiative Decays: Internal Conversion and Vibrational Relaxation ...... 23

1.2.3 Radiative Decays: Fluorescence ...................................................... 25

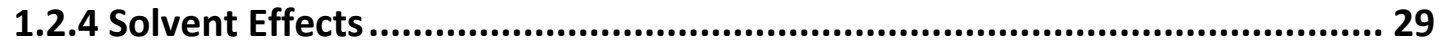

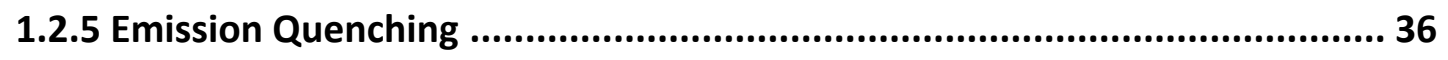

1.2.6 Non-radiative Relaxation Channel through Torsional Motions in Organic Materials.................................................................................................... 41

1.2.7 Hydrogen Bond Formation in Carboxylic Acid .......................................... 44

1.3 Materials and Experimental Methods .................................................... 46

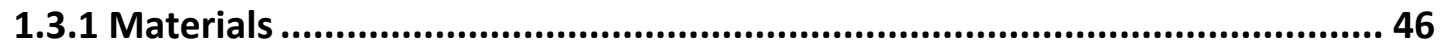

1.3.2 Experimental Methods ........................................................................ 48

1.3.2.1 Absorption Spectrometer ............................................................. 49

1.3.2.2 Spectrofluorometer.............................................................. 50 
1.3.2.3 Integrating Sphere Spectrophotometer .............................................. 51

1.3.2.4 Time-correlated Single Photon Counting .......................................... 53

1.3.2.5 Nuclear Magnetic Resonance .......................................................... 54

1.3.2.6 Mass Spectrometer ................................................................. 56

1.3.2.7 Density Functional theory (DFT) ....................................................... 57

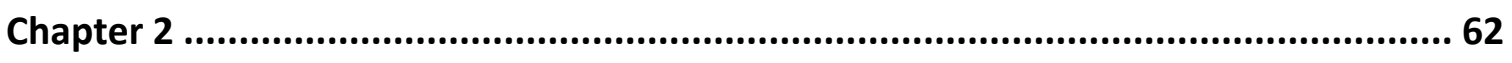

Spectroscopic Analysis of the Influence of Twisting Motion of a BTDB Molecule on its Photophysical Properties ................................................................................ 62

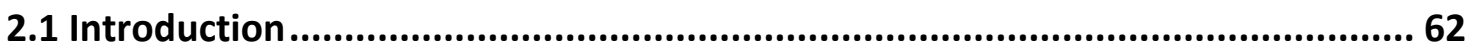

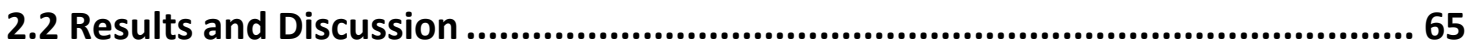

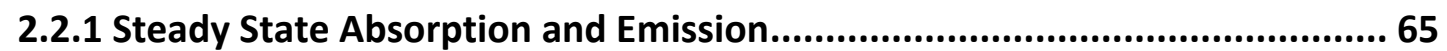

2.2.2 Density Functional Theory (DFT) Calculations ......................................... 68

2.2.3 Equilibrium Calculations ................................................................ 73

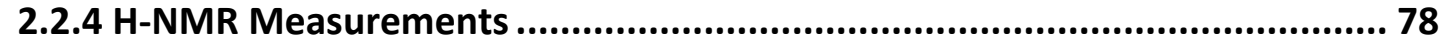

2.2.5 Deprotonation of Carbocyclic Acid Functionality .................................... 79

2.2.6 Photophysical Properties of Ester-form of BTDB Organic Molecule ............ 82

2.2.7 Excited State Lifetime and Emission Quantum Yield Measurements........... 83

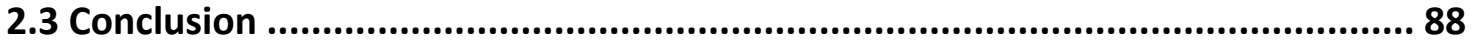

2.4 Future Perspective .................................................................................. 89

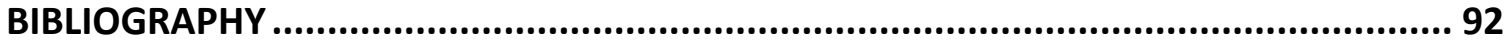




\section{LIST OF ABBREVIATIONS}

\begin{tabular}{|c|c|}
\hline AA & amino alcohol \\
\hline BTDB & $\begin{array}{l}\text { benzothiazole dicarboxylic derivative N, } N^{\prime} \text { - bis(terphenyl 4,4"- } \\
\left.\text { dicarboxylic acid) naphthalenediimide ( } \mathrm{H}_{4} \mathrm{BTD}-\mathrm{NDI}\right)\end{array}$ \\
\hline But & n-butanol \\
\hline CFD & constant function discriminator \\
\hline $\mathrm{CH}$ & cyclohexane \\
\hline DFT & density functional theory \\
\hline DMF & dimethylformamide \\
\hline DMSO & dimethyl sulfoxide \\
\hline DNS & 4-dimethylamino-4'-nitrostilbene \\
\hline EA & ethylacetate \\
\hline $\mathrm{EtOH}$ & ethanol \\
\hline fs-TA & femtosecond-transient absorption \\
\hline FWHM & full width at half maximum \\
\hline HOMO & highest occupied molecular orbital \\
\hline IC & internal conversion \\
\hline LUMO & lowest unoccupied molecular orbital \\
\hline MOF & metal organic framework \\
\hline NMR & nuclear magnetic resonance \\
\hline
\end{tabular}




$\begin{array}{ll}\text { PCBM } & \text { Phenyl-C61-butyric acid methyl ester } \\ \text { PCM } & \text { polarizable continuum model } \\ \text { PMMA } & \text { plastic poly (methyl methacrylate) } \\ \text { PLQY } & \text { photoluminescence quantum yield } \\ \text { TAC } & \text { time to amplitude converter } \\ \text { TCNE } & \text { tetrahydrofuran } \\ \text { THF } & \text { time-dependent density functional theory } \\ \text { TD-DFT } & \text { time resolved photoluminescence } \\ \text { TRPL } & \text { vibrational relaxation }\end{array}$




\section{LIST OF SYMBOLS}

S indicates a singlet electronic energy state

A absorption

$T \quad$ transmittance

I absorption intensity

$\varepsilon \quad$ molar absorptivity

$l \quad$ length of path traveled

$c_{i} \quad$ concentration of material $\mathrm{i}$ in molarity

$\phi \quad$ emission quantum yield

$k_{r} \quad$ radiative decay rate

$k_{n r} \quad$ non-radiative decay rate

$\tau \quad$ excited state lifetime

E energy

h Plank's constant

$f \quad$ frequency

c speed of light

$\lambda \quad$ wavelength

$\gamma \quad$ physical constant known as gyromagnetic ratio

$B \quad$ the magnitude of a magnetic field

$\pi \quad$ a mathematical constant that can be approximated to be 3.14 
$M \quad$ a unit of concentration, molarity

$\mu \quad$ micro, or $10^{-6}$

$n \quad$ nano, or $10^{-9}$

$m \quad$ a monomer species, a single unit

$D \quad$ a dimer species, two monomers bound together

$O \quad$ an oligomer species, a small number of monomers bound together

$K_{e q}^{i} \quad$ the equilibrium constant of formation of material $\mathrm{i}$

[i] indicates the concentration of material $\mathrm{i}$ in molarity 


\section{FIGURE CAPTIONS}

Figure 1. An illustration of a generic metal-organic framework structure along with its constituents: metal ions and organic linkers, adapted from ref. ${ }^{5}$

Figure 2. An illustration of the critical role of carboxylate functionality in chelating organic linkers to metals, represented by Cobalt in the image to build various 3dimentional frameworks, readapted from ref. ${ }^{11}$

Figure 3. An overview of Jablonski Energy Diagram showing a general picture of molecular electronic structure, along with relevant spectroscopic processes, readapted from ref. ${ }^{13}$

Figure 4. An illustration of Franck-condon principle that asserts that inter-nuclear distance does not change upon excitation due to the fast rate of the excitation process, readapted from ref. ${ }^{18}$

Figure 5. An illustration of Stokes shift reflecting the difference in wavelength and wavenumber between absorption and emission spectra, readapted from ref. ${ }^{21}$ 28

Figure 6. Schematic representation of intramolecular charge transfer from the perylene moiety to the carbonyl group upon the excitation of a formylperylene molecule at 450 $\mathrm{nm}$. The ground- and excited-state hydrogen-bond interaction in methanol is shown, readapted from ref. ${ }^{24}$

Figure 7. Steady-state absorption and emission spectra of formylperylene in various solvents: $\mathrm{CH}_{\mathrm{x}}$ (cyclohexane), $\mathrm{CAN}$ (acetonitrile), $\mathrm{MeOH}$ (methanol), and various binary mixtures of $\mathrm{ACN}$ and $\mathrm{MeOH}$, readapted rom ref. ${ }^{24}$

Figure 8. (A) Schematic representation of solute-solvent interaction in the ground state of the solute, and in the excited state upon excitation, readapted from ref. ${ }^{21}(B)$ time evolution of fluorescence of the solute upon excitation, readapted from ref. ${ }^{21}$

Figure 9. (A) An energy scheme showing energy loss as part of solvent dynamic process, readapted from ref. ${ }^{22}$ (B) An emission spectra scheme showing how polar solvents result in large Stokes shifts when compared with non-polar solvents, readapted from ref. ${ }^{22}$.. 36

Figure 10. (A) Steady-state absorption and emission of PbS-880 quantum dots upon the addition of various concentrations of Phenyl-C61-butyric acid methyl ester (PCBM), which acts as a quencher, readapted from ref. ${ }^{25}$ (B) Steady-state absorption and emission of PbS-1320 quantum dots upon the addition of various concentrations of Phenyl-C61-butyric acid methyl ester (PCBM), which acts as a quencher, readapted from ref. ${ }^{25}$ 
Figure 11. Steady-state absorption (left) and emission spectra (right) of methylammonium lead bromide perovskite nanocrystals of different shapes: (A) sphere, (B) platelet, and (C) cube, upon the successive addition of tetracyanoethylene (TCNE), readapted from ref. ${ }^{27}$ 39

Figure 12. (A) Kinetic traces of methylammonium lead bromide perovskite nanocrystals of different shapes, spheres, platelets, and cubes in the absence (cyan) and presence of TCNE (orange), readapted from ref. ${ }^{27}$ (B) Excited-state lifetime measurements of methylammonium lead bromide perovskite nanocrystals of different shapes, spheres, platelets, and cubes in the absence (cyan) and presence of TCNE (orange), readapted from ref. ${ }^{27}$

Figure 13. An illustrative scheme of hydrogen bonding interaction between two carboxylic acid groups.

Figure 14. Simplified scheme showing the synthesis of N, $N^{\prime}$ - bis(terphenyl-4,4"dicarboxylic acid) naphthalenediimide ( $\left.\mathrm{H}_{4} \mathrm{BTD}-\mathrm{NDI}\right)$, known as BTDB molecule.

Figure 15. (A) ${ }^{1} \mathrm{H}$ NMR spectra of $\mathrm{N}, \mathrm{N}^{\prime}$ - bis(terphenyl-4,4"-dicarboxylic acid) naphthalenediimide ( $\left.\mathrm{H}_{4} \mathrm{BTD}-\mathrm{NDI}\right)$ in DMSO, showing the aromatic hydrogen peaks.

(B)Zoom-in ${ }^{1} \mathrm{H}$ NMR spectra of N,N'- bis(terphenyl-4,4"-dicarboxylic acid) naphthalenediimide ( $\left.\mathrm{H}_{4} \mathrm{BTD}-\mathrm{NDI}\right)$ in DMSO. (C) Mass spectra of N, $\mathrm{N}^{\prime}$ - bis(terphenyl-4,4"dicarboxylic acid) naphthalenediimide ( $\mathrm{H}_{4} \mathrm{BTD}-\mathrm{NDI}$ ) in DMF. (D) TLC for $\mathrm{N}, \mathrm{N}^{\prime}-$ bis(terphenyl-4,4"-dicarboxylic acid) naphthalenediimide ( $\left.\mathrm{H}_{4} \mathrm{BTD}-\mathrm{NDI}\right)$ shows only one emitting dot under UV, thus confirming the purity of the sample......

Figure 16. An illustration of the main components of an absorption spectrometer, and how a signal is collected, readapted from ref. ${ }^{31}$

Figure 17. An illustration of how emission measurements are processed in a spectrofluorometer.

Figure 18. An illustration of emission spectra are processed in a typical time-correlated single photon counting device to quantify average lifetime values, readapted from ref. ${ }^{34}$

Figure 19. An illustration of nucleus quantization upon applying a magnetic field, readapted from ref. ${ }^{36}$

Figure 20. An overview of how a signal is produced in a mass spectrometer, readapted from ref. ${ }^{37}$

Figure 21. (A) Comparison of absorption spectrum of diphenylacetylene between experiment (black) and DFT-based calculation (red), readapted from ref. ${ }^{39}$ (B) 
Comparison of emission spectrum of diphenylacetylene between experiment (black) and DFT-based calculation (red), readapted from ref. ${ }^{39}$

Figure 22. (A) DFT-optimized geometry of dipehnylacetylene in the ground state, readapted from ref. ${ }^{39}$ (B) DFT-optimized geometry of dipehnylacetylene in the excited state, readapted from ref. ${ }^{39}$ (C) DFT-optimized geometry of 4-dialkylamino-2-

benzylidene malonic acid dimethyl ester in the ground state, readapted from ref. ${ }^{29}$ (D) DFT-optimized geometry of 4-dialkylamino-2-benzylidene malonic acid dimethyl ester in the excited state, readapted from ref. ${ }^{29}$

Figure 23. The chemical structure of the Benzothiazole dicarboxylic derivative (BTDB) organic molecule.

Figure 24. (A) Steady state absorption spectra of BTDB molecules in DMF at various concentrations in the range of 3-1000 $\mu \mathrm{M}$. (B) Emission spectra of BTDB molecules in DMF at various concentrations in the range of $0.1-64 \mu \mathrm{M}$.

Figure 25. Emission spectra of BTDB molecule in DMF in the concentration range of 64$1000 \mu \mathrm{M}$.

Figure 26. (A) Emission spectra of BTDB molecule at various concentrations in DMSO. (B) Emission spectra of BTDB molecule at various concentrations in EtOH. (C) Emission spectra of BTDB molecule at various concentrations in THF.

Figure 27. (A) Calculated absorption spectrum of BTDB in DMF, along with the optimized ground state structure and the dihedral angle, while showing the electronic density of HOMO and LUMO states. (B) Calculated emission spectrum of BTDB in DMF, along with the optimized excited state structure and the dihedral angle.

Figure 28. Calculated emission spectra of BTDB molecule in DMF with various dihedral angle between phenyl units.

Figure 29. Calculated emission spectra of monomer BTDB molecule, aggregate of two BTDB molecules through $\pi-\pi$ stacking, and dimer of BTDB molecules through hydrogenbond formation, along with optimized structure of each species and dihedral angles... 71

Figure 30. Calculated emission spectra of several BTDB-based linear oligomers: dimer, trimer, and tetramer.

Figure 31. A plot of the total emission intensity with respect to BTDB concentration,

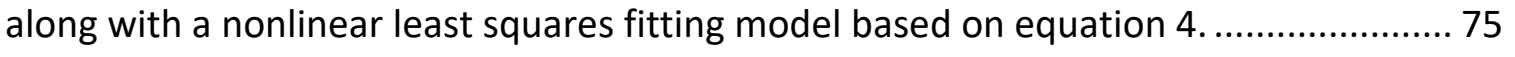

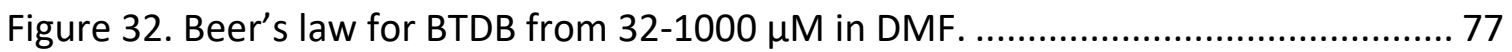


Figure 33. A plot of the total emission intensity with respect to BTDB concentration, along with a nonlinear least squares fitting model based on equation 8 .

Figure 34. H-NMR zoomed-in on the carboxylic acid region to show how FWHM is positively correlated with concentration of BTDB, and indicating extensive hydrogenbond formation when increasing the concentration.

Figure 35. H-NMR spectra showing the disappearance of the carboxylic acid peak upon the addition of an organic base to a BTDB solution.

Figure 36. (A) Normalized absorption spectra of 64 and $1000 \mu \mathrm{M}$ of linker in DMF in the absence and presence of organic base. (B) Scaled emission spectra of 64 and $1000 \mu \mathrm{M}$ of linker in DMF in the absence and presence of organic base, while showing the similarity between deprotonated species and the monomer species, taken to be $0.1 \mu \mathrm{M}$.

Figure 37. Normalized absorption and emission spectra of the ester-form of BTDB molecules, carboxylic acid functionality is replaced with ethyl ester, across three different concentrations.

Figure 38. Normalized time-resolved emission for TBDB linker in DMF at various concentrations.

Figure 39. (A) Normalized emission spectra of BTDB linker at various concentrations in PMMA. (B) TRPL for linker in DMF with $1 \mathrm{mM}$ concentration and low concentration embedded in PMMA.

Figure 40. A scheme featuring the potential energy diagrams vs. nuclear coordinates of all BTDB species discussed in the thesis: monomer, dimer, and oligomer species. 88 


\section{LIST OF TABLES}

Table 1. PLQY data of 4-dialkylamino-2-benzylidene malonic acid dimethyl ester in various solvents, along with their viscosity values, readapted from ref. ${ }^{29}$

Table 2. Photophysical data of Thioflavin in water/aerosol-OT media with different condiment indexes.Wo $=$ [water][aerosol-OT], reflecting the dispersion, or unconfinement, of the media, readapted from ref. ${ }^{28}$

Table 3. Dielectric constant and dipole moment numerical values of DMF, DMSO, EtOH, and THF 68

Table 4. Fluoresence-quantum yield data and excited state lifetime measurements, along with corresponding global fitting using exponential equation: A1e-t/ $\tau 1+$ $\mathrm{A} 2 \mathrm{e}-\mathrm{t} / \tau 2$ of BTDB at various concentrations in the presence and absence pf organic base 


\section{Chapter 1}

\section{Introduction}

\subsection{Organic Linkers in MOFs}

Organic compounds are used in a wide range of applications, such as fluorescent probes $^{1}$, super-molecular electronics ${ }^{2}$, and white-light generation ${ }^{3}$, as well as in various branches of science and engineering. In addition, organic molecules are essential building blocks of many chemical frameworks. Since the 1990s, metal-organic frameworks (MOFs) have been under development, and experienced an exponential growth in terms of research efforts and scope ${ }^{4}$. MOFs are forming a special class of chemical frameworks, where the overall structure incorporates both organic and inorganic components as shown in Figure 1. In a generic MOF, structure is made of nodes, represented by metal ions or clusters, that are interconnected via organic linkers 5 . Depending on the choice of metal ion or cluster, and organic linker as well as the way linkers bond to the positivelycharged molecule, distinct structures and shapes can be developed. To Build a MOF structure, several synthesis methodologies have been developed, such as hydrothermal and solvothermal techniques ${ }^{6}$. By examining MOF's frameworks, one can easily observe that one of the most valued feature of MOF structures is their high porosity, and how MOFs are considered some of the highest porous materials discovered ${ }^{5}$. In addition, the total surface area of a MOF material can reach to thousands of $\mathrm{m}^{2}$ per gram of material ${ }^{7}$. 


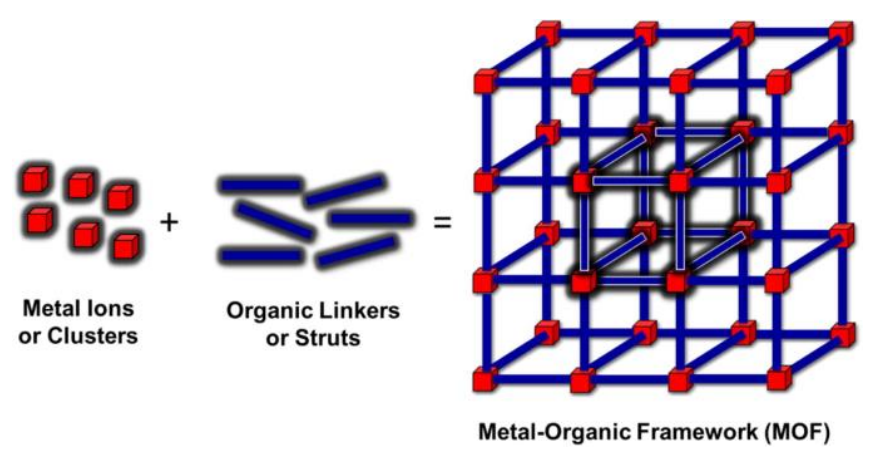

Figure 1. An illustration of a generic metal-organic framework structure along with its constituents: metal ions and organic linkers, adapted from ref. ${ }^{5}$

In addition, one can easily realize that several structures of MOF materials have been developed in different dimensionality and constituents ${ }^{8}$. The flexibility in structural design and choice of components makes this class of materials an ideal candidate to be utilized in various types of applications in science and engineering, such as catalysis, sensing and gas separation membranes ${ }^{8}$. To design MOF structures, organic linkers with multi-dentate carboxylate functionalities are utilized ${ }^{8}$. The carboxylate functionality is critical in chelating metal ions, and form $\mathrm{M}-\mathrm{O}-\mathrm{C}$ clusters to help build various 1,2 , or 3 dimenssional structures, see Figure $2^{8}$. Due to the way organic linkers are embedded within MOF structures, they are generally rigid, and their movements are restricted ${ }^{8}$. When comparing spectroscopic properties of a free organic linker with a linker embedded in a MOF structure, one may expect that their spectroscopic behaviors can be very different $^{9}$. Therefore, it is concluded that the environment surrounding the organic linker plays a vital role not only in controlling its optical properties, but also the structure rigidity of MOF materials. Moreover, the fixation of the incorporated organic linkers seem to greatly influence the photophysical properties of these organic molecules, such as emission spectrum, quantum yield and excited-state lifetime ${ }^{10}$. This environmental 
insight is critical when realizing that spectroscopic properties of an organic linker can be tuned through varying structure rigidity. Also, this feature can be very powerful upon the utilization of an organic linker in an application. Researchers have realized the wide flexibility they have when designing MOF structures, thus being able to control the rigidity of a structure, as well as the properties of system of interest.

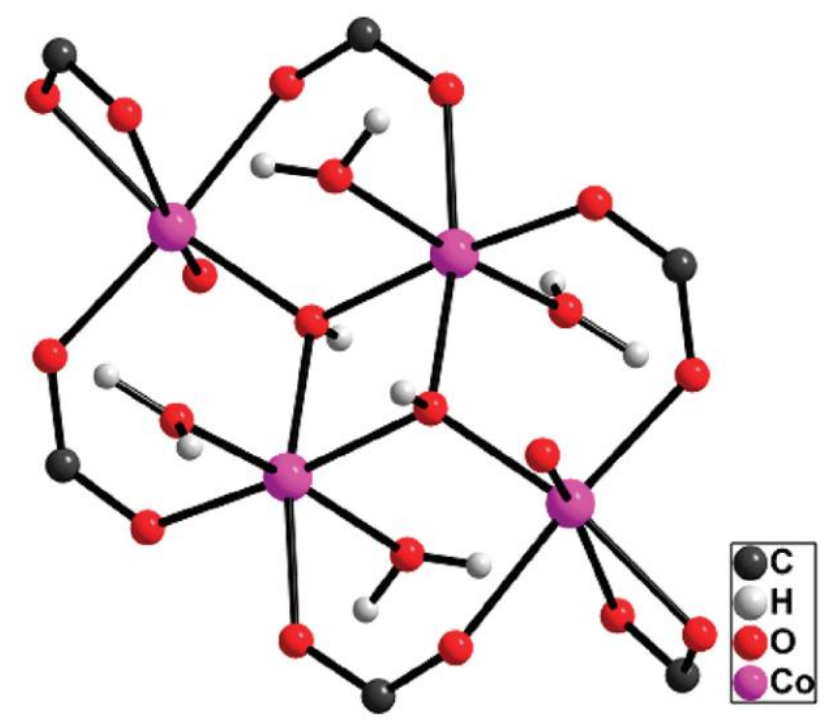

Figure 2. An illustration of the critical role of carboxylate functionality in chelating organic linkers to metals, represented by Cobalt in the image to build various 3-dimentional frameworks, readapted from ref. ${ }^{11}$

When considering structure rigidity of organic linkers embedded in a MOF material, several factors should be taken into account, such as length, structure, and torsional rigidity. Varying the length and 3-dimensional orientation of an organic linker can lead to significant changes in the physical and spectroscopic properties, such as porosity and emission properties, of the MOF of interest. Taken benzothiazole dicarboxylic derivative (BTDB) as a case in point, this study will aim to examine how 
restriction on movement is correlated with observed photophysical behavior of a certain organic molecule.

In chapter 1, an introduction to the main concepts of field of spectroscopy is presented, along with a brief description of some spectroscopic instruments used. Chapter 2 presents a full data analysis and discussion of how torsional motion of a BTDB molecule can greatly influence its spectroscopic properties.

\subsection{Excited-State Dynamics of Organic Molecules}

Chemical molecules have generally a very complex energy structure that determines the excited-state dynamic. Within each molecule, the structure is broken up into several electronic states: $S_{0}, S_{1}, S_{2}, S_{n}$, where $S_{0}$ has the lowest energy, and labeled as the ground state. $\mathrm{S}$ states correspond to a singlet quantum state. In addition to the $\mathrm{S}$ states, some molecules have a triplet state, labeled T state, that corresponds to a triplet quantum state where electron spins are not paired ${ }^{12}$. Initially, electrons occupy the lowest energy states to minimize the total molecular energy, and that the molecule is considered to be in the ground states. Energy separation between electronic states is usually significant, and requires the absorption of a photon with sufficient energy ${ }^{12}$. Within each electronic state, several sub-states exist that correspond to distinct vibrational modes ${ }^{12}$. Energy separation between vibration energy modes is small, and enough heating can have molecules move to higher vibration energy modes within an electronic state ${ }^{12}$. For electronic transitions, several photophysical processes can transfer an electron from a state to another. These processes include the following and not limited to: absorption, 
internal conversion and vibrational relaxation, intersystem crossing, phosphorescence, and fluorescence ${ }^{12}$. Spectroscopic processes occur at distinct timescales; excitation occurs within the timescale of one femtosecond while relaxation occurs within the timescale of sub-picosecond and fluorescence in the range of one nanosecond ${ }^{12}$. Figure 3 summarizes the general picture of molecular electronic structure, along with relevant transition processes.

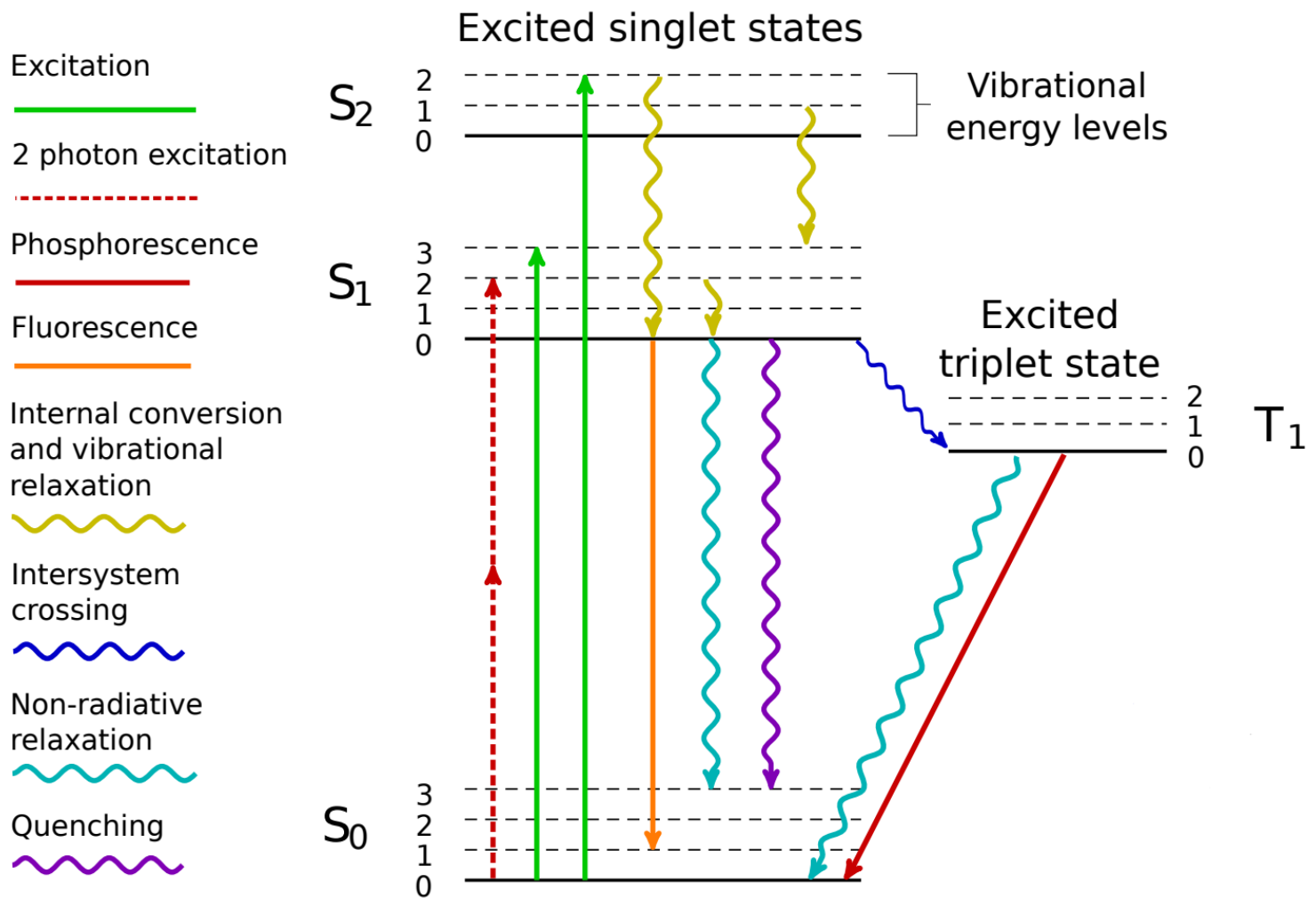

Figure 3. An overview of Jablonski Energy Diagram showing a general picture of molecular electronic structure, along with relevant spectroscopic processes, readapted from ref. ${ }^{13}$ 


\subsubsection{Steady-state Absorption}

Steady-state absorption involves a molecule absorbing a photon to excite an electron from the ground electronic state to higher states. For a transition to occur, the absorbed photon needs to provide sufficient energy to facilitate the transfer of an electron based on the energy separation between the ground state and the excited state of the system of interest. Furthermore, some electronic transitions are forbidden due to quantum mechanical constraints. For a transition to be allowed, the transition moment function has to be symmetric ${ }^{14}$. Examining allowed transitions, one finds that the absorption intensity depends on several factors: molecule's concentration, path length, and molar absorptivity (molar extinction coefficient) ${ }^{15}$. Intensity is generally formulated in a mathematical expression known as Beer-Lambert law as follows:

$$
A=-\log T=-\log \frac{I}{I_{0}}=\varepsilon \times l \times c_{i}
$$

Where $A$ is absorbanc, $T$ is transmittance, $I$ is the intensity of light leaving the sample, $I_{0}$ is the intensity of light entering the sample of interest, $\varepsilon$ is molar absorptivity, formally known as molar extinction coefficient, $l$ is the length of path traveled, and $c_{i}$ is the concentration of molecule of interest in molarity.

Therefore, it is deduced that the intensity of the absorption peak is directly proportional to the concentration of molecule of interest. Moreover, absorbing a photon is generally a very fast process, and occurs in the order of $10^{-15}$ second $^{12}$. Molecular 
absorption is generally characterized by the wavelength at which absorption is maximized.

\subsubsection{Non-radiative Decays: Internal Conversion and Vibrational}

\section{Relaxation}

After photon's absorption, the excited molecule can relax to the ground state again via different non-radiative processes such as an internal conversion (IC), or vibrational relaxation (VR). Generally, energy separation between adjacent excited states is small, and that there is an overlap between the potential energy surfaces of adjacent electronic excited states ${ }^{16}$. This overlap facilitates the release of energy through nonradiative mechanisms, and assist electrons to go to lower energy states ${ }^{16}$. This observation suggests that when an electron is excited to a high electronic excited state, it will release its excited energy through a non-emissive mechanism, most likely in the form of heat ${ }^{16}$. Once an electron goes through an IC, and reaches the $S_{1}$ excited state, it will relax to the ground state through a radiative process or a non-radiative process depending on energy separation and overlap of the potential energy surfaces of the relevant states. Typically, IC does not facilitate the transfer of an electron to the ground state since energy separation between $S_{1}$ and $S_{0}$ is generally significant, unless other mechanisms are involved such as torsional motions ${ }^{12}$. IC is generally a fast process, and occurs in the order of $10^{-14}-10^{-11}$ second ${ }^{12}$.

Due to the fast speed at which electronic changes occur, inter-nuclear distances stay constant through the change ${ }^{17}$. Due to the fixation of the inter-nuclear distance 
during the course of an electronic change, the vibrational energy level occupied at the final electronic state should have similar inter-nuclear distance to that of the vibrational energy level occupied at the initial electronic state, see Figure $4^{18}$. This phenomenon forms the basis of Franck-condon principle. Therefore, the vibrational energy level that an electron occupies once it reaches a state, may not correspond to the most optimized state. Therefore, upon the completion of an electronic excitation, a VR takes place accompanied with a corresponding change in inter-nuclear distance ${ }^{18}$. Typically, a VR is a process that occurs in the timescale of $10^{-12}$ second $^{18}$.

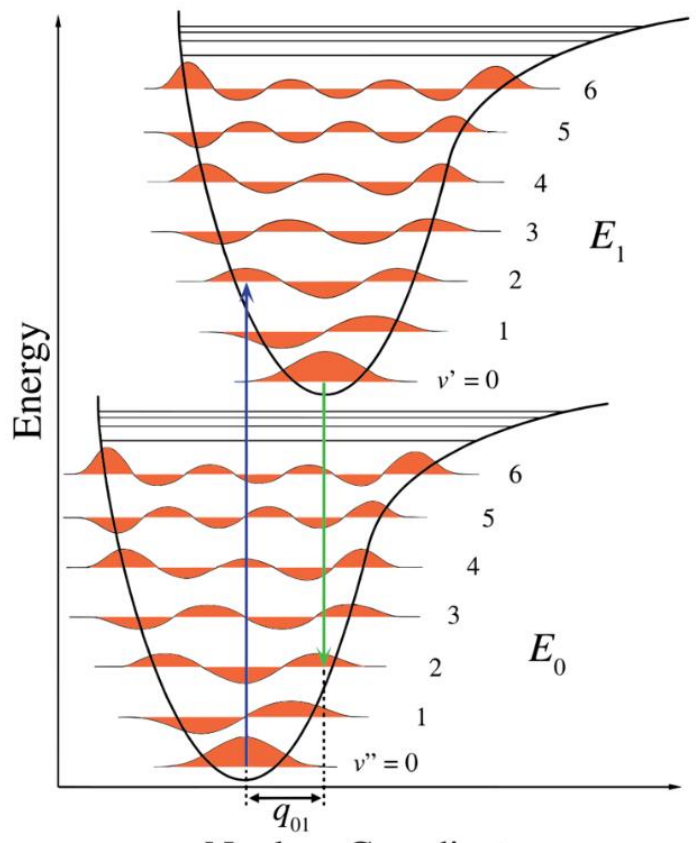

Nuclear Coordinates

Figure 4. An illustration of Franck-condon principle that asserts that inter-nuclear distance does not change upon excitation due to the fast rate of the excitation process, readapted from ref. ${ }^{18}$ 


\subsubsection{Radiative Decays: Fluorescence}

Once a molecule is excited, it can relax from its lowest excited state, $S_{1}$, to $S_{0}$ through emitting a photon. This observation forms the basis of Kasha's rule that states that upon light excitation, photon emission occurs from the lowest excited state, as known as $S_{1}$, to the ground state, labeled as $S_{0}{ }^{18}$. Kasha's rule is a very powerful rule in the field of spectroscopy, and leads to very important consequences. Firstly, the resulting emission will occur from the first excited electronic state, labeled as $S_{1}$, to the ground state, labeled as So regardless of the energy of the absorbed photon ${ }^{18}$. This is observed because even when a molecule is excited to higher energy states, labeled as $S_{n}$ where $n>$ 1 , it will quickly relax to $S_{1}$ non-radiatively through IC. Secondly, the ratio of molecules relaxing to the ground state through a radiative process to the total number of excited molecules, is an intrinsic property of the material of interest ${ }^{19}$. This ratio is known as photoluminescence quantum yield (PLQY), labeled as $\phi$. This ratio is important to quantify the number of excited molecules that relax to the ground state through radiative versus non-radiative decays. The PLQY of a molecule is defined as the ratio of number of emitted photons to the number of absorbed photons, and can be mathematically written as follows:

$$
\phi=\frac{\text { number of emitted photons }}{\text { nmber of absorped photons }}
$$

PLQY of a material depends solely on the rates of radiative and non-radiative decays, and can be expressed as 


$$
\phi=\frac{k_{r}}{k_{r}+k_{n r}}
$$

Where $k_{r}$ is the radiative rate constant, and $k_{n r}$ is the non-radiative rate constant.

There are several non-radiative processes that can facilitate molecules to go back to the ground state without emitting photons. Examples include intramolecular charge transfer, conformational change, torsional motions, electron and proton transfer, energy transfer, and excimer formation ${ }^{19} . k_{n r}$ encompasses the sum of the rate constants of all non-radiative processes ${ }^{19}$. Due to non-radiative relaxing processes, PLQY is always less than unity ${ }^{19}$. Therefore, due to the uniqueness of photoluminescence quantum yield (PLQY) of each material, its numerical value can be used to confirm the identity of a specific material. Thirdly, as a direct consequence of Kasha's rule, lifetime of the excited state is an intrinsic property of the material of interest, and generally denoted as $\tau$. The lifetime of the excited state is defined as the average time spent in the excited state for a molecule before releasing its energy, and decaying back to the ground state. Furthermore, the lifetime of the excited state depends of the rate constants of decay to the ground state via both emissive and non-emissive channels, and that the relationship can be shown to take on the following mathematical expression:

$$
\tau=\frac{1}{k_{r}+k_{n r}}
$$

Generally, fluorescence lifetimes of organic materials are in the timescale of $10^{-9}$ second $^{19}$. 
PLQY and lifetime of excited state of a substance are related due to their sole dependence on the rates of radiative and non-radiative decays; and they are correlated through the following mathematical expression:

$$
\begin{gathered}
\phi=\frac{\tau}{\tau_{n}} \\
k_{r}=\phi / \tau \text {, then } k_{n r}=(1-\phi) / \tau
\end{gathered}
$$

In addition to PLQY and lifetime of the excited state, emission can be characterized by the wavelength at which maximum emission intensity is observed. Generally, when comparing emission peak to absorption peaks, one finds that absorption peak always occurs at a shorter wavelength than the wavelength at which the emission peak locates. This wavelength difference is generally denoted as Stokes shift, see Figure $5^{20}$. 


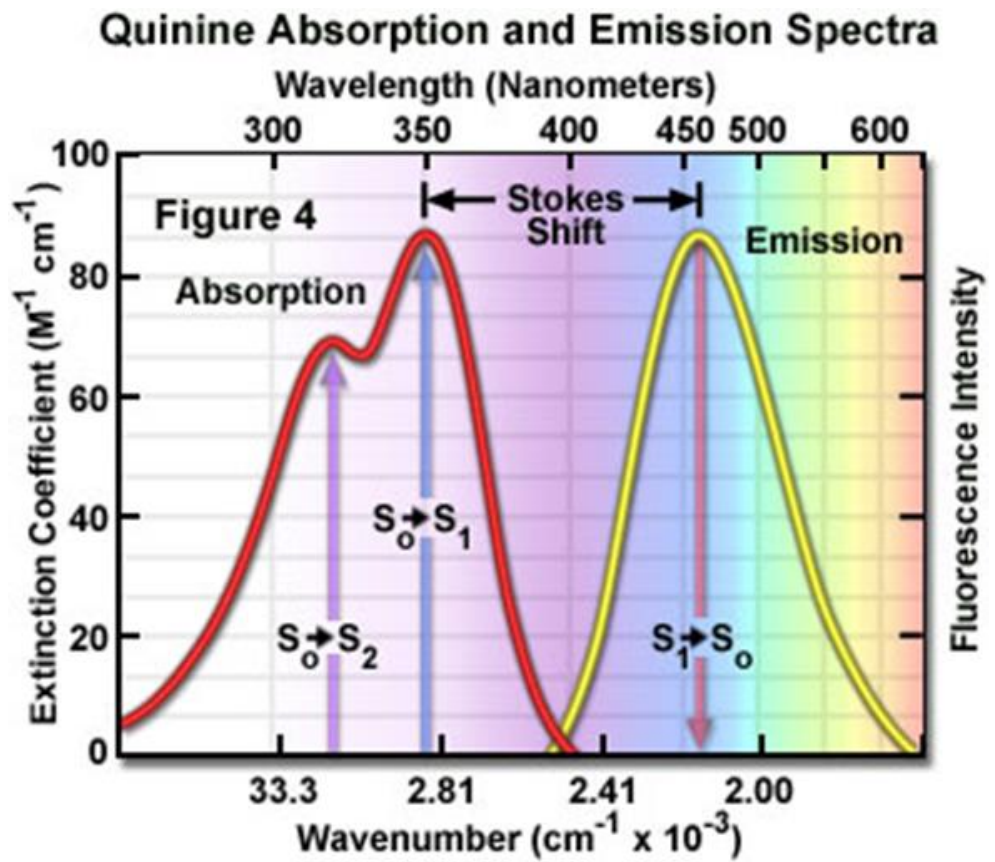

Figure 5. An illustration of Stokes shift reflecting the difference in wavelength and wavenumber between absorption and emission spectra, readapted from ref. ${ }^{21}$

Stokes shift can be easily unraveled when converting photon wavelengths to their respective energy values. It is known that a photon's energy depends solely on frequency and wavelength as follows:

$$
E=h f=\frac{h c}{\lambda}
$$

Where $E$ is photon's energy, $h$ is Plank's constant, $f$ is photon's frequency, $c$ is speed of light, and $\lambda$ is photon's wavelength.

Due to internal conversion, vibrational relaxation, and solvation dynamic processes that molecules go through in solution before going back to the ground state, one finds that absorption peak corresponds to a higher energy than that of emission peak $^{19}$. The mentioned processes in solution correspond to loss of energy as molecules 
go to lower excited electronic states, which explains the notion why emission energy tend to correspond to lower energy than absorption.

\subsubsection{Solvent Effects}

Since chapter 2 discusses spectroscopic properties of an organic compound in solution, it is important to discuss the effect of solvent on how the excited molecule behaves in such environment from a spectroscopic perspective. When a molecule is dissolved in a solvent, it surrounds itself with solvent molecules, and interacts with them accordingly. This solute-solvent interaction leads to profound results in the spectroscopic properties, especially absorption, emission and photoluminescence quantum yield, of the solute. There are number of factors that influence absorption, emission and photoluminescence quantum yield. These factors include solvent polarity and viscosity, rate of solvent relaxation, rigidity of local environment, internal charge transfer, excited state-proton transfer, and changes in radiative and non-radiative decay rates ${ }^{22}$. However, when considering solvent effects, solvent polarity and hydrogen bond capabilities are typically the most influential factor, and will be discussed in detail in this section ${ }^{22}$.

Typically, the location of the absorption peak depends on the type of solvent used $^{23}$. In the presence of a highly polar solvent, electrostatic interaction and hydrogen bonding dominate the type of interaction involved between the fluorophore and solvent molecules, especially upon the presence of functional groups in the fluorophore, such as carboxylic acid, ketone, or hydroxyl groups ${ }^{23}$. These are considered high energy interactions, and will result in the absorption peak to be located at lower wavelengths ${ }^{23}$. 
On the other hand, in the presence of a low polarity solvent, interaction between the fluorophore and solvent molecules is relatively weak, and will generally result in absorption at higher wavelengths compared to the polar ones ${ }^{23}$.

Solvent polarity and hydrogen-bonding formation are also common drivers that control solvent-solute interaction, and influence solute's spectral and dynamical behaviors. For example, the photophysical properties of formylperylene has been investigated, and observed spectral behavior is shown to be heavily influenced by the used solvent. Zewail and co-workers have shown that upon excitation of formylperylene molecule at $450 \mathrm{~nm}$, an intramolecular charge transfer from the perylene moiety to the carbonyl group takes place as illustrated in Figure $6^{24}$. Steady-state absorption and emission spectra have been collected in polar and nonpolar media, as well as protic and aprotic media to illustrate the effect of the solvent polarity and hydrogen bonding interactions on the observed spectral and dynamical behaviors of formylperylene. 


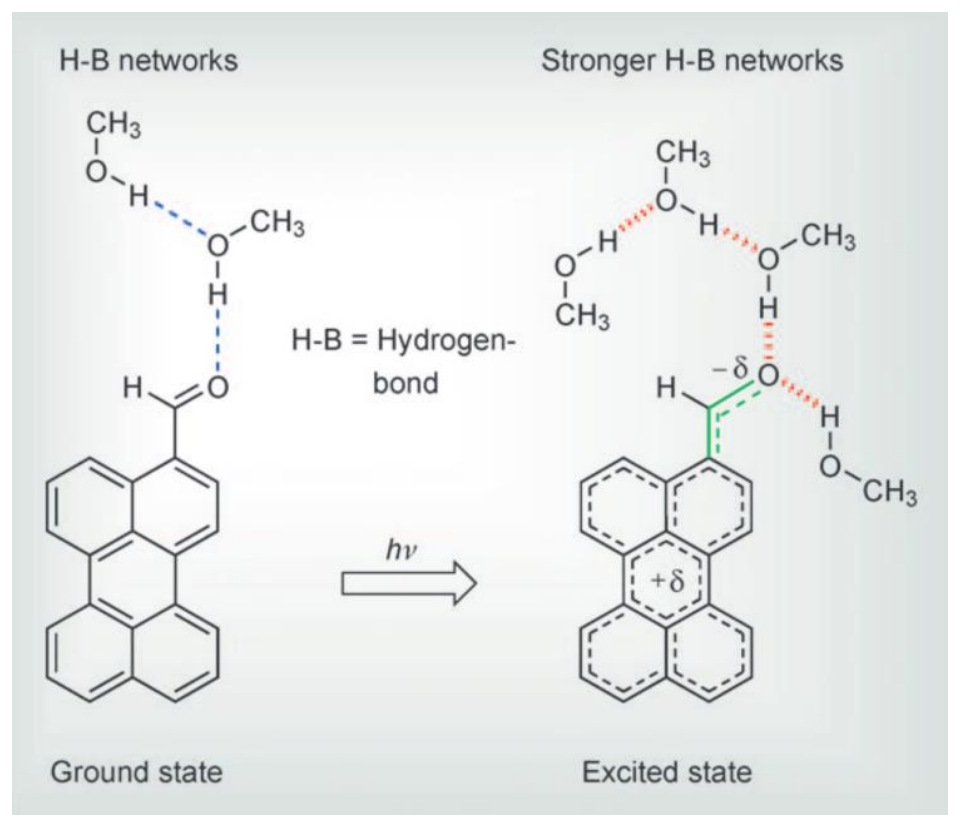

Figure 6. Schematic representation of intramolecular charge transfer from the perylene moiety to the carbonyl group upon the excitation of a formylperylene molecule at $450 \mathrm{~nm}$. The ground- and excited-state hydrogen-bond interaction in methanol is shown, readapted from ref. ${ }^{24}$

Figure 7 shows that the absorption and emission spectra of formylperylene in cyclohexane, acetonitrile, methanol, and various binary mixtures of both acetonitrile and methanol. Looking at absorption spectra in binary mixtures, there is a systematic change in the red-tail when increasing the concentration of methanol. On the other hand, examining emission spectra, one observes that the mirror symmetry between absorption and emission spectra is broken when changing the media from nonpolar to polar, namely from cyclohexane to acetonitrile or methanol, and that emission spectra become featureless and broad in polar media. In addition, because of the intramolecular charge transfer from the perylene moiety to the carbonyl group, it is expected that the electron density around the carbonyl group will be enhanced upon excitation. Therefore, it is projected that the carbonyl group will be more prone to participating in hydrogen- 
bonding interaction, and act as a hydrogen bond acceptor in protic solvents. Figure 6 shows that a schematic representation of the enhancement of hydrogen bonding interaction between formylperylene and methanol upon excitation. To measure the influence of hydrogen-bonding interaction on the spectral behavior of formylperylene, emission spectra of formylperylene have been obtained in acetonitrile, methanol, and various binary mixtures of both. Acetonitrile and methanol have similar polarity, while acetonitrile is considered aprotic and methanol to be protic ${ }^{24}$. Looking at Figure 7, it is clearly shown that there is a systematic red-shift in emission upon increasing the concentration of methanol in the binary mixture, thus an enhancement to the size of Stokes shift is observed. 


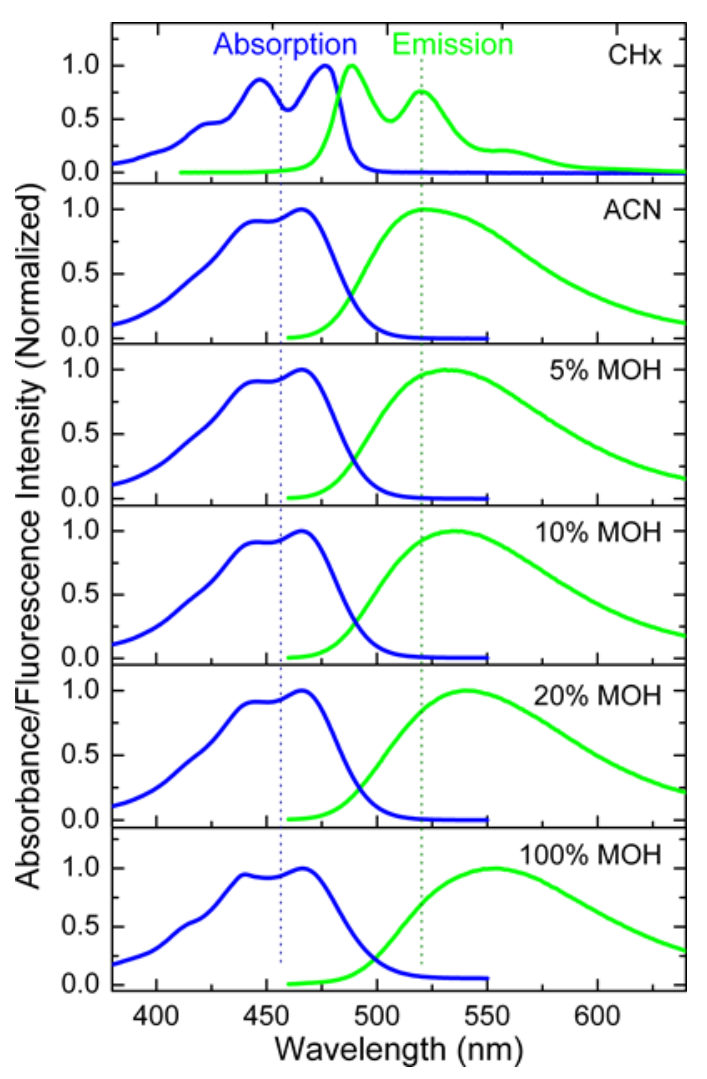

Figure 7. Steady-state absorption and emission spectra of formylperylene in various solvents: $\mathrm{CH}_{X}$ (cyclohexane), $\mathrm{CAN}$ (acetonitrile), $\mathrm{MeOH}$ (methanol), and various binary mixtures of $A C N$ and $\mathrm{MeOH}$, readapted rom ref. ${ }^{24}$

As mentioned previously, fluorophores emit photons at longer wavelengths than the wavelength at which absorption occurs. Examining how the magnitude of stokes shift of a molecule changes in solution, one finds that the magnitude of stokes shift is a function of the solvent used as well, see Figure $9^{22}$. Furthermore, there is a systematic correlation between the size of stokes shift and the polarity of the solvent used, in which the excited fluorophore has different dipole moment compared to the ground state as illustrated in the previous example 22,24 . Typically, the dipole moment of the excited fluorophore will be larger than when it is in the ground state ${ }^{22}$. Therefore, following excitation, due to the change in the dipole moment of the solute, solvent molecules will 
reorient themselves to relax around the new dipole moment ${ }^{22}$. In other words, before excitation, the solute is in the ground state, and it is orienting itself around solvent molecules in a way to minimize dipole-dipole interaction energy. Upon excitation, the solute's dipole moment will be changed. However, due to the fast process of photon absorption, in the order of 1 femtosecond, solvent molecules will not be able to reorient themselves to minimize dipole-dipole interaction energy within the same timescale. Therefore, upon excitation, the excited solute's energy will not correspond to the minimum energy on the excited state potential curve as shown in Figure $8(A)$. However, when monitoring the time evolution of the system, one observes that solvent molecules are able to reorient themselves to minimize energy and move along the potential energy curve to eventually reach the minimum again as shown in Figure 8 (A). Therefore, monitoring the time evolution of fluorescence spectra, one observes that there is a systematic red-shift as the system evolves, which is in full agreement with the described energy relaxation process. This process is known as solvation dynamic or relaxation, and generally occurs in the timescale of $10^{-10}$ second ${ }^{22}$. 

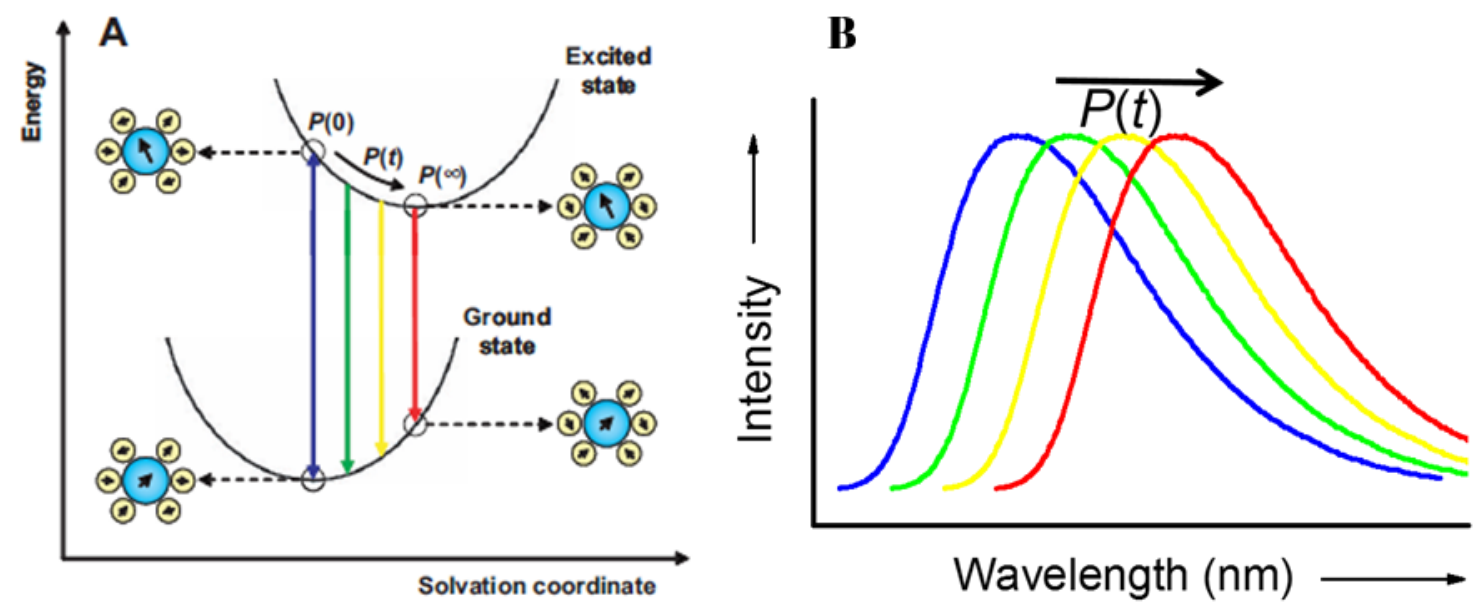

Figure 8. (A) Schematic representation of solute-solvent interaction in the ground state of the solute, and in the excited state upon excitation, readapted from ref. ${ }^{21}$ (B) time evolution of fluorescence of the solute upon excitation, readapted from ref. ${ }^{21}$

As the polarity of the solvent is increased, the effect will be larger, and will correspond to a more significant relaxation, see Figure $9^{22}$. Therefore, as the polarity of the solvent is increased, solvation dynamic will be more significant, and will result in a substantial stokes shift, see Figure $9^{22}$. One can clearly observe that the stokes shift of 4dimethylamino-4'-nitrostilbene (DNS) is more significant when using the following solvents in order: n-butanol (Bu), ethyl acetate (EA), toluene (T), cyclohexane ( $\mathrm{CH})$, hexane $(H)$, respectively ${ }^{22}$. This order is in perfect agreement with the order of increased polarity of the mentioned solvents ${ }^{22}$. However, it is important to note that solvation dynamic is important for polar fluorophores, and that nonpolar fluorophores are insensitive to the polarity of solvent used ${ }^{22}$. 

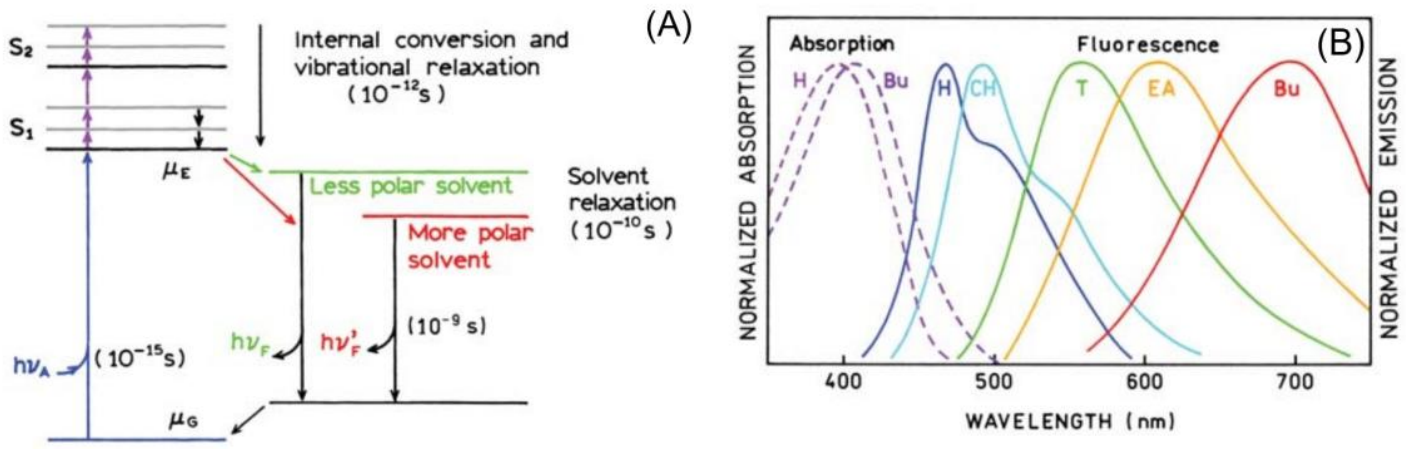

Figure 9. (A) An energy scheme showing energy loss as part of solvent dynamic process, readapted from ref. ${ }^{22}$ (B) An emission spectra scheme showing how polar solvents result in large Stokes shifts when compared with non-polar solvents, readapted from ref. ${ }^{22}$

\subsubsection{Emission Quenching}

Fluorescence quenching refers to any process that results in a drop in the emission intensity of a material upon the addition of a second material. Figure 10 shows how Phenyl-C61-butyric acid methyl ester (PCBM) acts as a quencher to the photoluminescence of $\mathrm{PbS}-880$ and $\mathrm{PbS}-1320$ quantum dots as a result of electron transfer between excited PbS quantum dots and PCBM molecules ${ }^{25}$. Various chemical processes can lead to a drop in the emission intensity of a sample ${ }^{26}$. Molecular interaction between the material of interest and quencher forms the basis of such chemical processes $^{26}$. These processes include the following: excited-state electron transfer, molecular rearrangements, energy transfer, excited state complex formation, and collisional quenching ${ }^{26}$. There are two general categorical mechanisms at which quenching processes occur ${ }^{26}$. The two mechanisms are called dynamic quenching and static quenching ${ }^{26}$. 

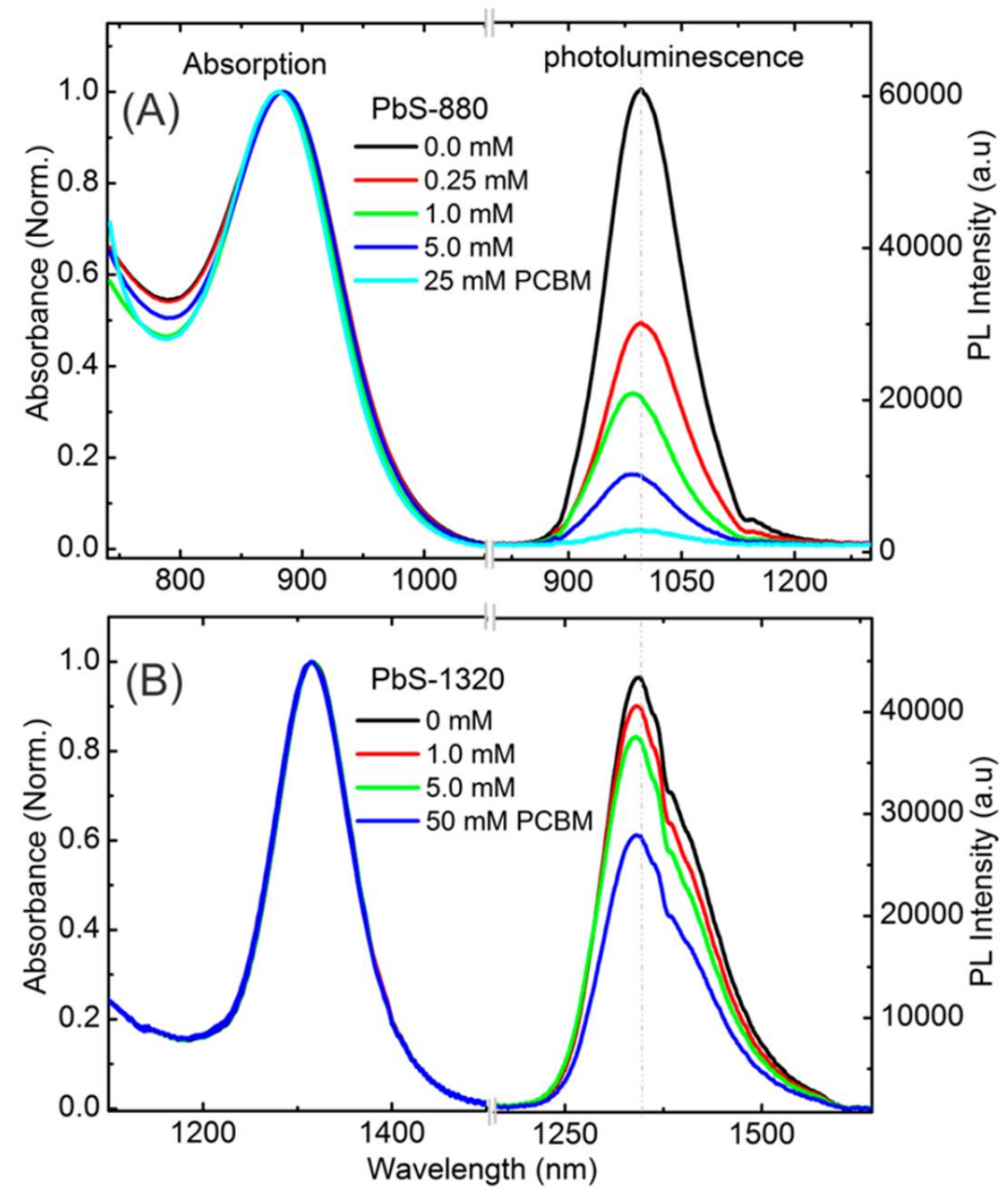

Figure 10. (A) Steady-state absorption and emission of PbS-880 quantum dots upon the addition of various concentrations of Phenyl-C61-butyric acid methyl ester (PCBM), which acts as a quencher, readapted from ref. ${ }^{25}$ (B) Steady-state absorption and emission of PbS1320 quantum dots upon the addition of various concentrations of Phenyl-C61-butyric acid methyl ester (PCBM), which acts as a quencher, readapted from ref. ${ }^{25}$

Dynamic quenching is sometimes labeled as collisional quenching or long distance excited state reactions ${ }^{26}$. In this scheme, the fluorophore returns back to the ground state through a diffusive mechanism with the quencher ${ }^{26}$. It is important to note that the molecule of interest is not chemically modified over the course of dynamic quenching ${ }^{26}$. There are several molecules that act as dynamic quenchers, but quench emission differently depending on how they interact with the fluorophore ${ }^{26}$. For example, when 
working with electron deficient quenchers, quenching can be facilitated by the transfer of an electron from the fluorophore to the quencher ${ }^{26}$.

On the other hand, static quenching occurs when fluorophores are transformed to non-fluorescent complexes ${ }^{26}$. In this mechanism, the molecule of interest reacts with the quencher to form a complex when it is in the ground state. Once the complex is formed, fluorophore-complexes become non-fluorescent, and relax to the ground state upon excitation through non-radiative channels. Static quenching differs from dynamic quenching in three main ways. First, in static quenching, fluorophores are deactivated in complex formation, as opposed to dynamic quenching where the process occurs when fluorophores and quenchers are separated from each other ${ }^{26-27}$. Second, Static quenching does not depend on diffusion of either the fluorophore or the quencher to happen, while dynamic quenching relies heavily on the diffusional behavior of the excited state ${ }^{26-27}$. Third, the diffusion quenching rate is concentration dependent but static is not. 


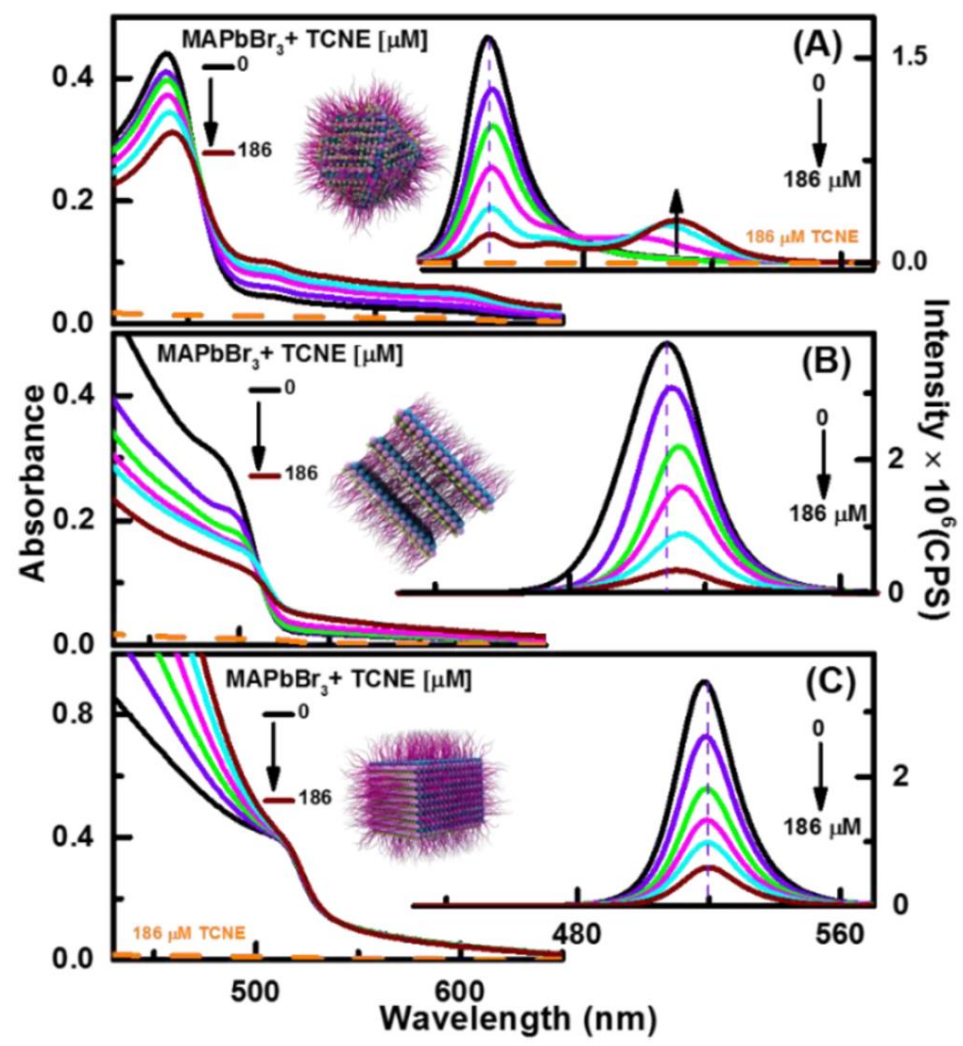

Figure 11. Steady-state absorption (left) and emission spectra (right) of methylammonium lead bromide perovskite nanocrystals of different shapes: (A) sphere, (B) platelet, and (C) cube, upon the successive addition of tetracyanoethylene (TCNE), readapted from ref. ${ }^{27}$

For example, quenching of methylammonium lead bromide perovskite nanocrystals of different shapes has been investigated. It has been shown that adding tetracyanoethylene (TCNE) quenches the photoluminescence of the perovskite nanocrystals as shown in Figure 11. Across all three shapes (sphere, cube, and platelet), one observes that the emission intensity is reduced upon increasing the concentration of TCNE added ${ }^{27}$. To help determine the mechanism at which the quenching process occurs, several tests have been carried out. Figure 11 shows that steady-state measurements without and with the addition of the quencher, reveal that there are spectral changes in the case of sphere and platelets, thus indicating the formation of new species when 
adding TCNE. In the case of spherical nanocrystals, Figure $11(\mathrm{~A})$ shows that new peaks emerge upon the addition of the quencher, which suggests that a complexation process has taken place ${ }^{27}$. Similarly, in the case of platelets nanocrystals, Figure $11(B)$ shows that a spectral shift has taken place upon the addition of the quencher, thus indicating the occurrence of a complexation process ${ }^{27}$. The complexation process in the case of spheres and platelets indicate that the quenching process is static. On the other hand, in the case of cubes, Figure 11 (C) shows that no spectral change is observed upon the addition of the quencher, thus suggesting that there is no complexation, and that quenching does not happen through a static mechanism ${ }^{27}$.

Furthermore, excited-state lifetime measurements have been conducted on the three different shapes of methylammonium lead bromide perovskite nanocrystals. In the case of spheres, Figure 12 shows that excited-state lifetime at longer time scale remains identical in the absence and presence of TCNE, thus indicating that the quenching occurs through a static mechanism ${ }^{27}$. On the contrary, for platelets and cubes, excited-state lifetime is decreased upon the addition of TCNE, thus suggesting that quenching occurs through a dynamic mechanism ${ }^{27}$. Therefore, when combining data from both femtosecond-transient absorption and excited-state decay of the three different shapes of methylammonium lead bromide perovskite nanocrystals, one concludes that sphereshaped nanocrystals are quenched via static mechanism, while platelets-shaped nanocrystals are quenched via both static and dynamic mechanisms. On the other hand, cube-shaped nanocrystals are quenched via a dynamic mechanism. 


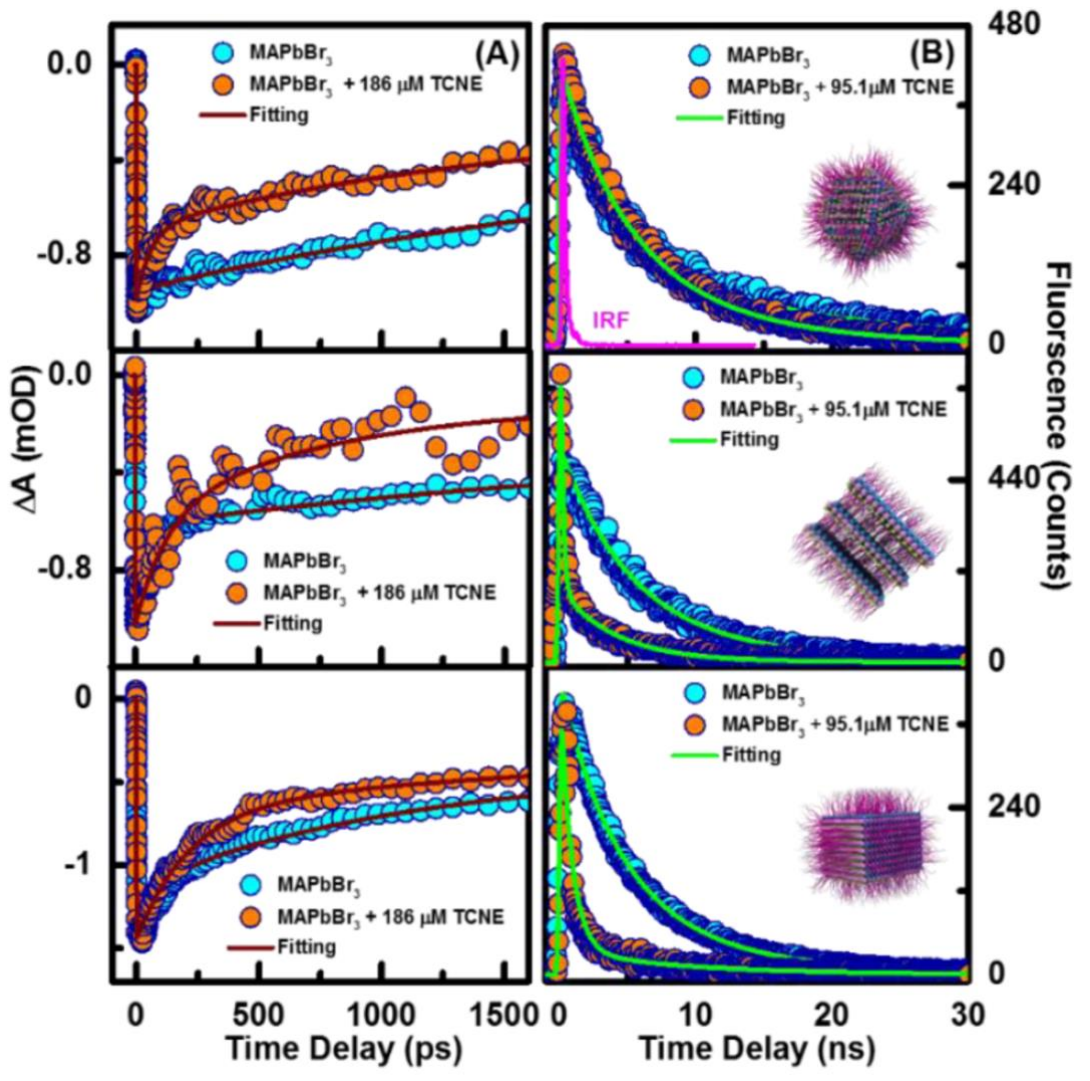

Figure 12. (A) Kinetic traces of methylammonium lead bromide perovskite nanocrystals of different shapes, spheres, platelets, and cubes in the absence (cyan) and presence of TCNE (orange), readapted from ref. ${ }^{27}$ (B) Excited-state lifetime measurements of methylammonium lead bromide perovskite nanocrystals of different shapes, spheres, platelets, and cubes in the absence (cyan) and presence of TCNE (orange), readapted from ref. ${ }^{27}$

\subsubsection{Non-radiative Relaxation Channel through Torsional Motions in}

\section{Organic Materials}

As mentioned previously, upon photon excitation, molecules decay back to the ground state through two main schemes: radiative and non-radiative processes. Examining and deciphering the non-radiative processes, one finds that torsional motion is one of the common radiation-less mechanisms for organic molecules ${ }^{28-29}$. Rotational 
conformational change demands that the molecule of interest to be flexible to go through a torsional motion in the excited state. In other words, highly rigid molecules where any form of torsional motion may collapse the structure, will not go through rotational conformational changes. For example, highly cyclic molecules tend to be extremely rigid, and that any torsional motion is highly restricted to take place. The occurrence of torsional motions can induce significant changes on the photophysical properties of organic molecules ${ }^{28}$. Torsional motion tends to provide a fast pathway for molecules to decay back to the ground state without emitting a photon ${ }^{28}$. Therefore, excited state life time and photoluminescence quantum yield (PLQY) will drop significantly as molecular torsional motion takes place ${ }^{28}$.

The presence of torsional-motion-based non-radiative channel in organic molecules has been verified experimentally, as well as its influence on observed photopysical properties ${ }^{28-29}$. To assess torsional motions of a molecule, researchers have attempted to restrict the mentioned motions by varying the viscosity of the media, as well as the usage of confined water poo ${ }^{28-29}$. It is well-known that viscosity is a measure of the resistance of relative motion between the media and the material of interest. Therefore, it is expected that when increasing the viscosity of the media, the molecular torsional motion will be suppressed, and that the PLQY will increase. For example, a study has been carried out on 4-dialkylamino-2-benzylidene malonic acid dimethyl ester, a molecule that undergoes molecular torsional motion upon excitation ${ }^{29}$. Table 1 shows how PLQY varies with solvents of different viscosities for 4-dialkylamino-2-benzylidene malonic acid 
dimethyl ester. It is shown that upon increasing the viscosity of the media by 243 folds, PLQY was increased by 26 folds, thus confirming the suppressing of molecular torsional motions ${ }^{29}$.

Table 1. PLQY data of 4-dialkylamino-2-benzylidene malonic acid dimethyl ester in various solvents, along with their viscosity values, readapted from ref. ${ }^{29}$

\begin{tabular}{|c|c|c|}
\hline Solvent & Viscosity of solvent (cP) & PLQY \\
\hline acetonitrile & 0.37 & $<0.001$ \\
\hline dichloromethane & 0.44 & 0.001 \\
\hline diethylene glycol & 35.7 & 0.003 \\
\hline polyethylene glycol & 90 & \\
\hline
\end{tabular}

Furthermore, a study has been performed on Thioflavin, a molecule that undergoes molecular torsional motion upon excitation, to show that the mentioned motion is suppressed upon controlling the confinement of the media through the usage of a mixture of water and aerosol-OT ${ }^{28}$. When increasing the confinement of the media, Table 2 shows that torsional motion becomes sluggish, and that PLQY and excited state lifetime are enhanced accordingly due to the suppression of the torsional-motion-based nonradiative channel ${ }^{28}$. On the other hand, as the confinement of the media is lowered, torsional motion is increased, and that it corresponds to a drop in PLQY and excited state lifetime ${ }^{28}$. Therefore, it is concluded that torsional-motion-based non-radiative is a common mechanism at which molecules relax back to the ground state upon excitation. 
Table 2. Photophysical data of Thioflavin in water/aerosol-OT media with different condiment indexes. $W_{o}=\frac{[\text { water }]}{[\text { aerosol-OT] }}$, reflecting the dispersion, or unconfinement, of the media, readapted from ref. ${ }^{28}$

\begin{tabular}{|c|c|c|c|}
\hline$W_{o}$ & $k_{\text {tor }} \times 10^{-10}\left(s^{-1}\right)$ & $\phi$ & Average lifetime $(p s)$ \\
\hline 1 & 0.59 & 0.0255 & 166.0 \\
\hline 2 & 0.81 & 0.0155 & 121.4 \\
\hline 4 & 1.65 & 0.0059 & 60.3 \\
\hline 6 & 2.64 & 0.0039 & 37.8 \\
\hline 8 & 4.18 & 0.0029 & 23.8 \\
\hline 10 & 5.15 & 0.002 & 19.4 \\
\hline
\end{tabular}

\subsubsection{Hydrogen Bond Formation in Carboxylic Acid}

Hydrogen bond is a partially electrostatic interaction between a hydrogen atom that is bonding to an atom with high electronegativity value, namely fluorine, oxygen and nitrogen, and an adjacent atom with an electron lone pair. Due to the electronegativity difference, hydrogen atoms bonded to the highly electronegative atom, will bear a partial positive charge, and act as a hydrogen bond donor ${ }^{30}$. On the other hand, an atom with a lone pair of electrons will be electrostatically attracted to the partially positively-charged hydrogen atom, thus acting as a hydrogen bond acceptor ${ }^{30}$. Although exact bond energy values cannot be obtained experimentally, theoretical calculations have estimated hydrogen bond energy to be in the range of $0.2-40 \mathrm{kcal} / \mathrm{mol}^{30}$. Hydrogen bond formation is a broad phenomenon that is observed in a wide variety of chemical systems; hydrogen bonds can be intermolecular or intramolecular. 
Due to the presence of a carbonyl group along with an alcohol group adjacent to each other, carboxylic acid-based molecules are the best illustrative of hydrogen bonding interaction. The hydrogen of the carboxylic acid group acts as a hydrogen bond donor. The mentioned hydrogen carries a partial positive charge due to the adjacent oxygen atom. On the other hand, the oxygen of the carbonyl group acts as a hydrogen bond acceptor given that it has two available lone pairs. Furthermore, since each carboxylic acid group can act as a hydrogen bond donor, acceptor, or both, two hydrogen bonds can be formed when two carboxylic acid groups interact, see Figure 13. Hydrogen bond interaction between two distinct carboxylic acid groups is estimated to be about 15 $\mathrm{kcal} / \mathrm{mol}^{30}$.

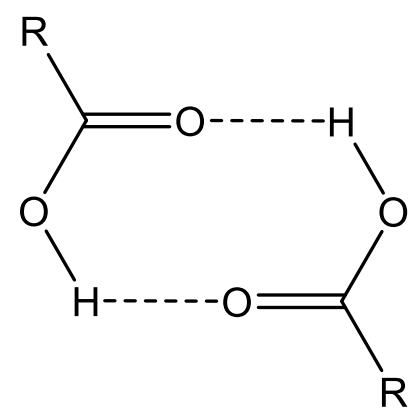

Figure 13. An illustrative scheme of hydrogen bonding interaction between two carboxylic acid groups. 


\subsection{Materials and Experimental Methods}

\subsubsection{Materials}

Dimethylformamide (DMF), ethanol (EtOH), Tetrahydrofuran (THF), and Dimethyl sulfoxide (DMSO) were purchased from Aldrich, and used without further purification. The synthesis procedure of BTDB linker, and ester-form of BTDB are outlined below.

Preparation of $N, N^{\prime}$ - bis(terphenyl-4,4"-dicarboxylic acid) naphthalenediimide ( $\mathrm{H}_{4}$ BTD-NDI): $40 \mathrm{~mL}$ of $\mathrm{CH}_{3} \mathrm{CN}$ was poured in a $250 \mathrm{~mL}$ round-bottom flask sealed with septum. Then, the flask was evacuated and backfilled three times with argon, and bubbling the solvent with argon for $1.5 \mathrm{~h}$ to ensure that an inert atmosphere is obtained. 1,3-Dibromo-anilene (1.83 g; $10 \mathrm{mmol}$ ), 4-carboxyphenylboronic acid (3.66 g; $22 \mathrm{mmol}$ ), $5 \% \mathrm{Pd}\left(\mathrm{PPh}_{3}\right)_{2} \mathrm{Cl}_{2}(0.4 \mathrm{~g})$ and $40 \mathrm{~mL}$ aqueous potassium carbonate $(5.3 \mathrm{~g} ; 80 \mathrm{mmol})$ solution were added to the flask. Flask was then evacuated and backfilled three times with argon, and heated at $100{ }^{\circ} \mathrm{C}$ for $48 \mathrm{~h}$ while stirring the mixture. The mixture was then cooled down to room temperature, diluted with $200 \mathrm{~mL}$ of water, and filtered. The filtrate was acidified to $\mathrm{pH}=1$ using $2 \mathrm{~N} \mathrm{HCl}$, washed with water and hexane, and left to dry on air before drying the sample at high vacuum at $50{ }^{\circ} \mathrm{C}$ overnight to result in $3.11 \mathrm{~g}$ of white powder of anilene-3,5-dibenzoicacid. Then, 1,4,5,8-tetracarboxydianhydride $(0.268 \mathrm{~g}, 1.0$ $\mathrm{mmol}$ ) was out into a $250 \mathrm{~mL}$ round bottomed flask and suspended in $25 \mathrm{~mL}$ acetic acid. The mixture was stirred for few minutes before anilene-3,5-dibenzoicacid $(0.698 \mathrm{~g}, 2.2$ mmol) was added. The mixture was allowed to reflux for $12 \mathrm{~h}$. The reaction was allowed to cool down to room temperature before water $(90 \mathrm{~mL})$ was added to induce 
precipitation of the product. Finally, the product was collected by filtration, and washed with EtOH and dried in vacuum to result in $2.4 \mathrm{~g}$ of white powder. Finally, the final product was recrystallized from DMF as a yellow powder. Figure 14 outlines the synthesis scheme.

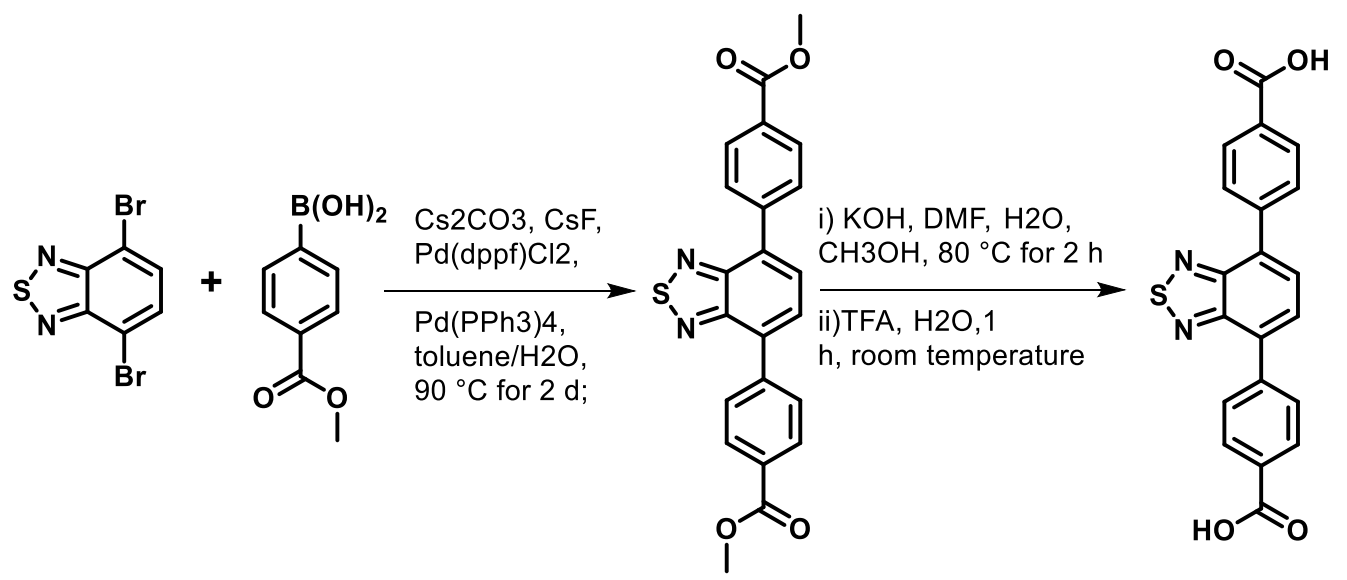

Figure 14. Simplified scheme showing the synthesis of $\mathrm{N}, \mathrm{N}^{\prime}$ - bis(terphenyl-4,4"dicarboxylic acid) naphthalenediimide ( $\left.\mathrm{H}_{4} \mathrm{BTD}-\mathrm{NDI}\right)$, known as BTDB molecule.

The identity and purity of $\mathrm{N}, \mathrm{N}^{\prime}$ - bis(terphenyl-4,4"-dicarboxylic acid) naphthalenediimide ( $\mathrm{H}_{4} \mathrm{BTD}-\mathrm{NDI}$ ) was verified using ${ }^{1} \mathrm{H}$ and ${ }^{13} \mathrm{C} N M R$, mass spectra, and TLC spectra, see Figure 15. outlines ${ }^{1} \mathrm{H} N M R$, mass spectra, and TLC spectra. For NMR, ${ }^{1} \mathrm{H}$ NMR ( $400 \mathrm{MHz}$, DMSO) $\delta=13.04(\mathrm{~s}, 4 \mathrm{H}), 8.73(\mathrm{~s}, 4 \mathrm{H}), 8.16(\mathrm{~s}, 2 \mathrm{H}), 8.05(\mathrm{~d}, \mathrm{~J}=8.1,8 \mathrm{H}), 7.97(\mathrm{~s}, 4 \mathrm{H}), 7.92(\mathrm{~d}, \mathrm{~J}=8.2$, $8 \mathrm{H}) .{ }^{13} \mathrm{C}$ NMR (400 MHz, DMSO) $\delta=167.06,162.97,162.29,143.02,140.65,137.09$, 130.57, 130.12, 130.01, 127.40, 127.04, 126.92, 126.56. HR-MS (ESI, [M-H]'): calcd for $\left[\mathrm{C}_{54} \mathrm{H}_{29} \mathrm{~N}_{2} \mathrm{O}_{12}\right]^{-:}: 897.17260$; found: 897.17424. 
(A)

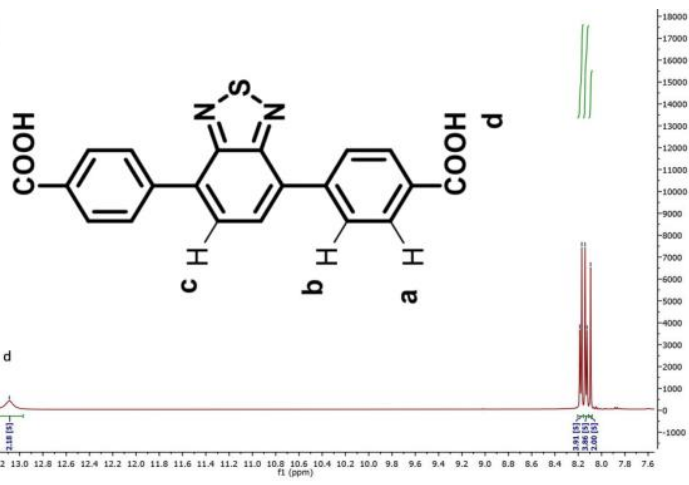

(B)

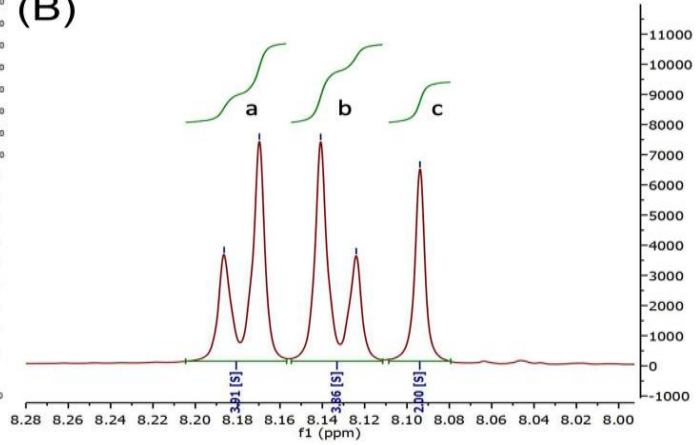

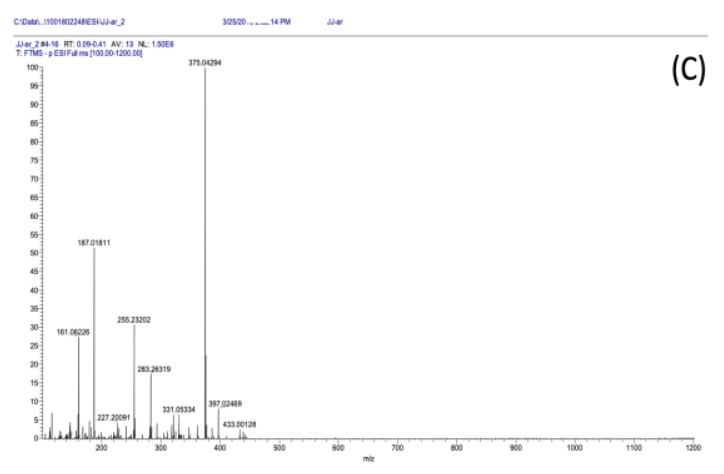

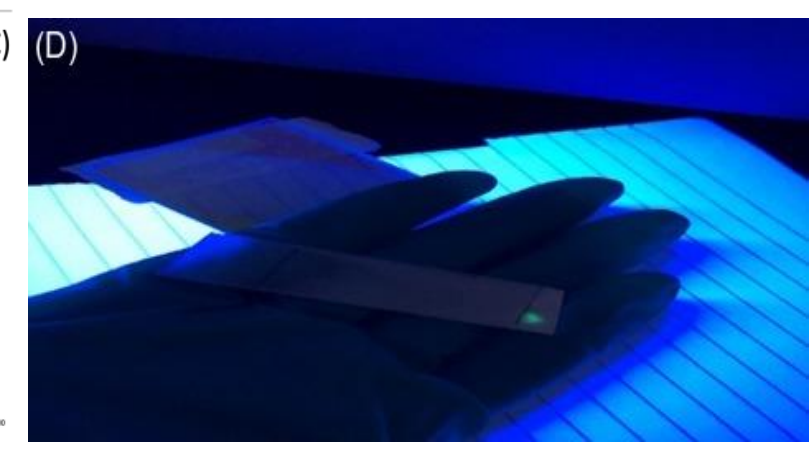

Figure 15. (A) ${ }^{1} H$ NMR spectra of $N, N^{\prime}$ - bis(terphenyl-4,4"-dicarboxylic acid) naphthalenediimide ( $\left.H_{4} B T D-N D I\right)$ in DMSO, showing the aromatic hydrogen peaks. (B)Zoom-in ${ }^{1} H \quad N M R$ spectra of $N, N^{\prime}$ - bis(terphenyl-4,4"-dicarboxylic acid) naphthalenediimide ( $\left.H_{4} B T D-N D I\right)$ in DMSO. (C) Mass spectra of $N, N^{\prime}$ - bis(terphenyl-4, $4^{\prime \prime}-$ dicarboxylic acid) naphthalenediimide ( $\left.H_{4} B T D-N D I\right)$ in DMF. (D) TLC for $N, N^{\prime}-$ bis(terphenyl-4,4"-dicarboxylic acid) naphthalenediimide ( $\left.H_{4} B T D-N D I\right)$ shows only one emitting dot under UV, thus confirming the purity of the sample.

\subsubsection{Experimental Methods}

Several instruments and techniques have been developed to analyze the excited state dynamic processes. Each technique sheds light on some of the details of a certain spectroscopic process. For example, an absorption spectrometer can uncover a sample's absorption behavior; while a spectrofluorometer can reveal the sample's emission behavior. When combining information obtained from multiple instruments, one can 
easily understand how the excited state dynamics of the sample of interest occurs. Below is a brief description of some of the major spectroscopic techniques utilized in chapter 2 as part of the experimental study.

\subsubsection{Absorption Spectrometer}

An absorption spectrometer is generally considered the workhouse of any spectroscopic lab, and is the first instrument to be used when analyzing the optical properties of a material ${ }^{31}$. It is typically used to obtain the absorption spectrum of a sample of interest. In an absorption measurement, the sample of interest is exposed to light with various different wavelengths, while measuring absorption. Absorption is defined as follows:

$$
A=-\log T=-\log \frac{I}{I_{0}}
$$

Where $A$ is absorption, $T$ is transmittance, $I$ is intensity of light leaving the sample, $I_{0}$ is intensity of light leaving the sample of interest.

Moreover, when collecting data points, a spectrometer measures the absorption of the material while scanning light's wavelength. To help isolate the absorption of the material of interest from that of other absorbents, an absorption measurement is always carried out of a blank sample. The blank sample should have all constituents that the sample of interest has in identical proportions, except for the constituent to be analyzed. All samples are generally measured in quartz cuvettes as part the sample preparation.

Figure 16 shows a very simplified illustration of how absorption measurement is 
processed in a spectrometer ${ }^{31}$. First, a source emits light, and a dispersion device is used to separate the emitted light into components of different wavelengths. Then, an exit slit is used to selectively let the light with the wavelength of interest pass to excite the sample. Once sample molecules are excited, a detector is used to measure emission, and generate a photoluminescence spectrum.

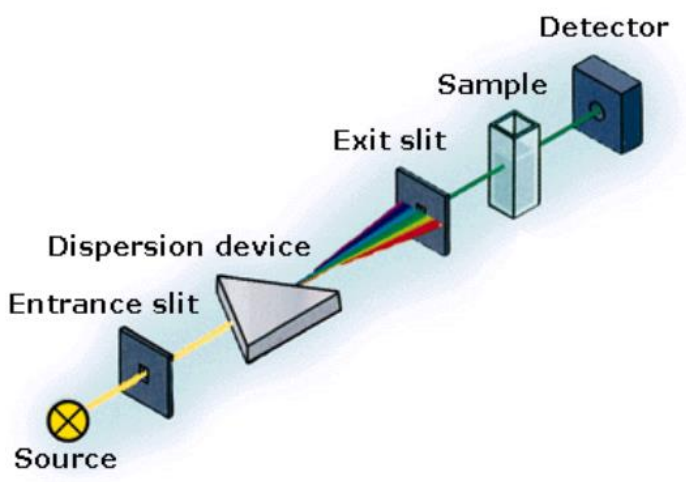

Figure 16. An illustration of the main components of an absorption spectrometer, and how a signal is collected, readapted from ref. ${ }^{31}$

\subsubsection{Spectrofluorometer}

Spectrofluorometers are a class of instruments utilized to measure the emission spectra of several types of materials ${ }^{32}$. In a typical emission measurement, the sample of interest is excited at a specific wavelength based on the material's absorption peak. In this study, excitation is done at the wavelength that corresponds to the maximum absorption in the absorption spectrum of the sample of interest. Upon excitation, the spectrofluorometer measures emission by scanning the number of emitted photons against a predefined range of wavelengths, leading to an emission spectrum. 
A typical spectrofluorometer consists of the following components: a light source, an excitation monochromator, a sample holder, an emission monochromator, and a detector $^{32}$. Figure 17 shows a very simplified illustration of how emission measurement is processed in a spectrofluorometer.

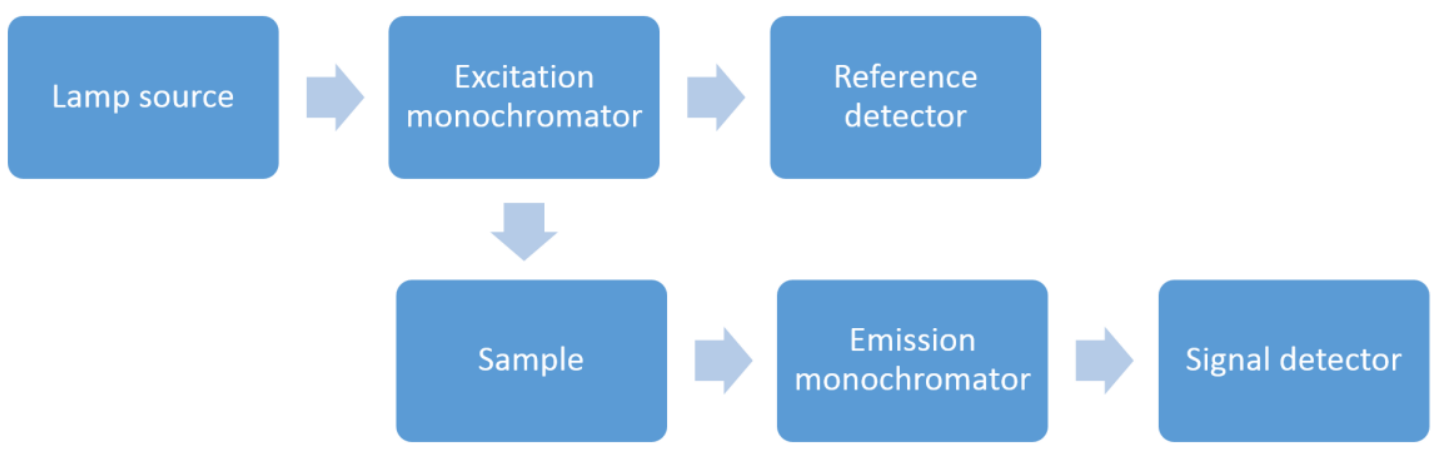

Figure 17. An illustration of how emission measurements are processed in a spectrofluorometer.

As shown in Figure 17, a lamp source is used to emit light, and that a monochromator is used to selectively excite the sample with the wavelength of interest. Then, upon molecular excitation and emission, a monochromator and a detector are used to generate an emission spectrum of the sample. To avoid accounting for the contribution of excitation light in the emission spectrum, a reference detector is added to the scheme to measure light passing through the excitation monochromator.

\subsubsection{Integrating Sphere Spectrophotometer}

Integrating sphere spectrophotometers are powerful tools utilized in spectroscopy to measure photoluminescence quantum yield of a variety of different materials. The preference to use integrating sphere spectrophotometers stems from their 
ease of use and accuracy ${ }^{33}$. As mentioned previously, photoluminescence quantum yield can be formulated mathematically ${ }^{19}$ as follows:

$$
\phi=\frac{\text { number of emitted photons }}{\text { nmber of absorped photons }}
$$

Therefore, to quantify the photoluminescence quantum yield of a material, the number of absorbed photons and the number of emitted photons have to be determined. To measure the number of photons, integrating spheres have been developed to spatially integrate radiant flux ${ }^{33}$. Several parameters influence the operation of an integrating sphere that include sphere multiplier and average reflectance ${ }^{33}$.

To identify the number of absorbed photons, the sample of interest is excited at the relevant wavelength, and the number of non-absorbed photons is recorded using the integrating sphere. Then, a blank sample is prepared that constitutes all components that the sample of interest has, except for the constituent to be analyzed. The blank sample is excited at the same wavelength, and the number of non-absorbed photons is recorded using the integrating sphere. Using a simple subtraction operation, one can quantify the number of absorbed photons by the constituent of interest. On the other hand, to quantify the number of emitted photons, emission of the sample is measured, and the number of emitted photons is recorded using the integrating sphere. Once both numbers are identified, photoluminescence quantum yield can be directly calculated. 


\subsubsection{Time-correlated Single Photon Counting}

In time-correlated single photon counting experiment, one can easily measure lifetime of excited state of a molecule to a high degree of accuracy, ${ }^{34}$ and lifetimes as low as 100 pico-second can be measured. In a typical measurement, it is aimed that one emitted photon is detected per laser pulse $\mathrm{e}^{34}$. However, in practice, many laser pulses result in photons not being detected, and that one photon is detected per 100 Laser pulses on average ${ }^{34}$. The accuracy of a measurement depends heavily on the arrival of emitted photons to the detector at various time intervals ${ }^{34}$.

In a typical measurement, upon the excitation by a laser pulse, two parallel simultaneous electrical signals are sent ${ }^{34}$. To measure time accurately, one of the two signals is used as a reference, while the other is related to the excitation and emission of a photon in the sample. Regarding the reference signal, the signal goes through a constant function discriminator (CFD), and then through time to amplitude converter (TAC) ${ }^{34}$. This signal reflects the start time indicator ${ }^{34}$. Looking at the sample signal, after the signal from the emitted photon passes through a CFD, it goes through the same TAC of the reference signal $^{34}$. This signal reflects the stop time indicator ${ }^{34}$. Once accounted for time delay in the electronics, a histogram plot is produced, and is translated into a familiar-looking lifetime plot $^{34}$. Figure 18 shows a very simplified illustration of how emission lifetime measurement is processed in a typical time-correlated single photon counting device ${ }^{34}$. 


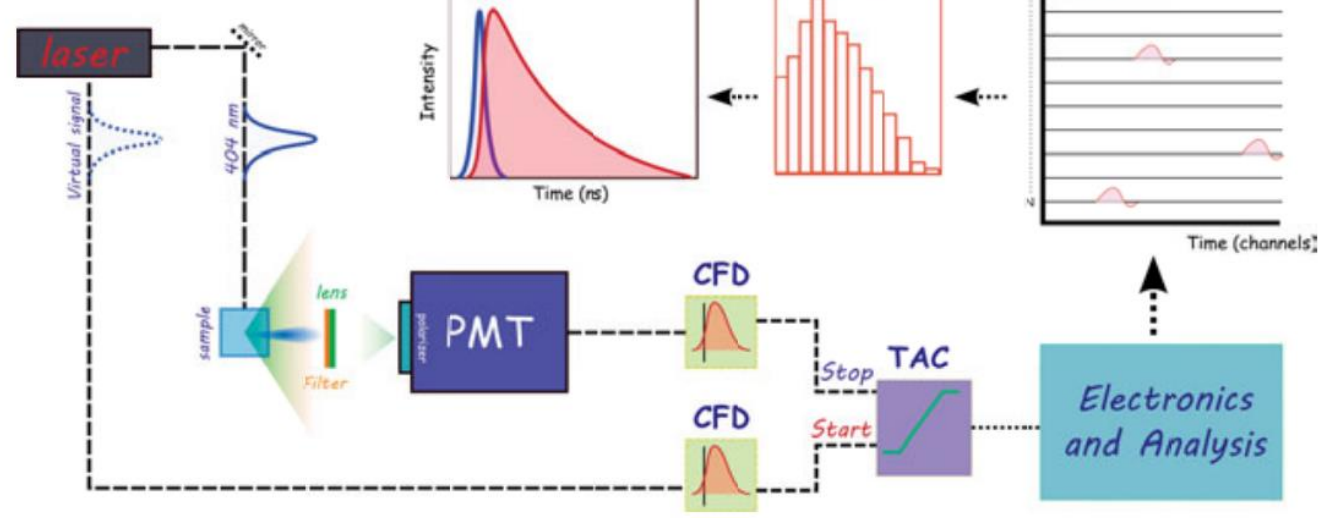

Figure 18. An illustration of emission spectra are processed in a typical time-correlated single photon counting device to quantify average lifetime values, readapted from ref. ${ }^{34}$

\subsubsection{Nuclear Magnetic Resonance}

Nuclear Magnetic Resonance (NMR) is one of the most powerful tools developed in the twentieth century, and has facilitated the advancements of several frontiers in science and engineering, especially chemistry. NMR is heavily used to determine and verify the identity and structure of unknown chemical compounds. The NMR technique revolves around the idea that a nucleus' spin can be changed upon the absorption of a photon with the right amount of energy under the influence of a magnetic field. For the technique to work, relevant nuclei need to have multiple nuclear states that they can occupy $^{35}$. When a nucleus has one spin state, NMR cannot be used since no spin excitation can occur, and that no information can be drawn. Generally, all isotopes that encompass an odd number of protons, neutrons, or both, are NMR active ${ }^{35}$. In other words, the mentioned nuclei have a nonzero spin number, and therefore they have more than one 
nuclear state ${ }^{35}$. Most useful NMR active nuclei have a spin number of $1 / 2$, which corresponds to two spin states: up " $\alpha$ " and down " $\beta$ ", see Figure $19^{36}$.

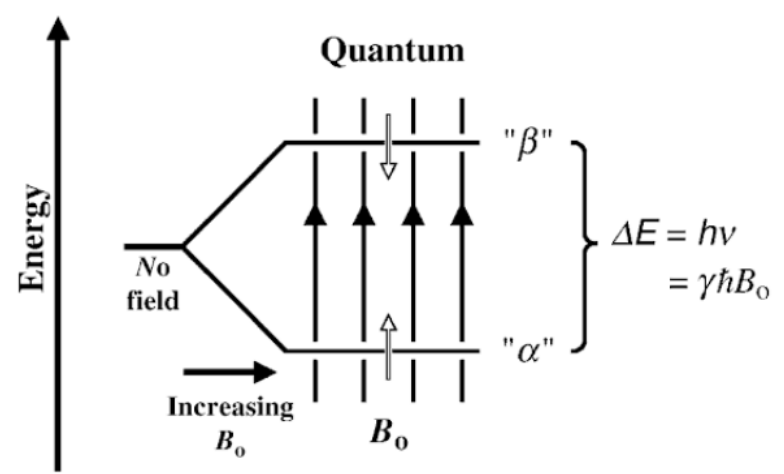

Figure 19. An illustration of nucleus quantization upon applying a magnetic field, readapted from ref. ${ }^{36}$

However, it is important to note that the two states have identical energies in the absence of a magnetic field, and that the energy split occurs once the nuclei are exposed to a magnetic field ${ }^{36}$. The $\alpha$ state, where the spin is aligned with the magnetic field, is more stable than the $\beta$ state, where the spin is opposed to the magnetic $\operatorname{field}^{36}$. The energy separation between the two states is proportional to the applied magnitude of the magnetic field, and is formulated mathematically as follows:

$$
\Delta E=\frac{h \gamma B_{o}}{2 \pi}
$$

Where $\Delta E$ is the energy gap between the two spin states, $h$ is Plank's constant, $\gamma$ is the gyromagnetic ratio, and $B_{o}$ is the magnitude of the applied magnetic field ${ }^{36}$

In the presence of a large population of nuclei, there will be an equilibrium between the two states, and that more than half of the nuclei will generally occupy the $\alpha$ 
state $^{36}$. This imbalance in nuclei occupation of the two spin states, along with the energy separation forms the basis of NMR.

Energy separation is not only a characteristic of the type of nucleus, but is also influenced by the electronic environment of the nucleus, since nearby electrons generate magnetic fields of their own that perturb the magnitude of the applied magnetic field, thus changing the energy separation between the spin states slightly ${ }^{36}$. Therefore, each chemical compound has a corresponding NMR spectrum that is unique, and can be utilized for identification purposes.

\subsubsection{Mass Spectrometer}

Mass spectrometer is one of the powerful tools used in analytical chemistry to gain quantitative and qualitative information about various substances, especially organic molecules. The analysis is based on measuring the mass of the molecule of interest, along with monitoring how it fragments upon the bombardment of high energy electrons. Examining the technical details of a mass spectrometer, one finds that the sample is first vaporized, and then ionized using high energy electrons according to the following equation:

$$
\text { Molecule }+e^{-} \rightarrow \text { Molecule }{ }^{+}+2 e^{-37}
$$

After ionization, molecules are accelerated by an electric field into a narrow beam. Once molecules are moving at a fast rate, a magnetic field is applied, and at which molecules

will be deflected based on their mass and charge ${ }^{37}$. Then, based on the amount of 
deflection, a detector is able to quantify the mass of arrived particles ${ }^{37}$. See Figure 20 for an overview of the described process.

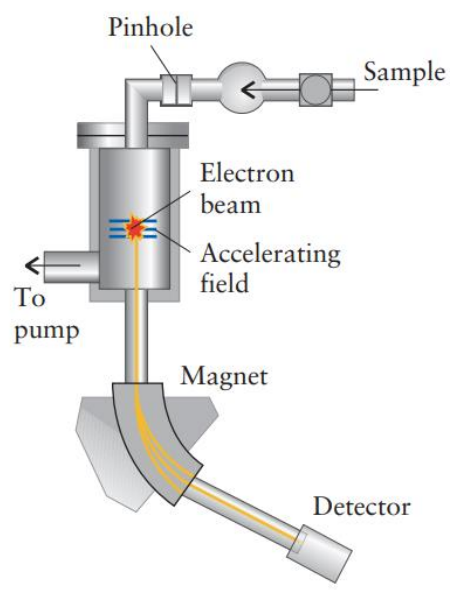

Figure 20. An overview of how a signal is produced in a mass spectrometer, readapted from ref. ${ }^{37}$

Furthermore, during the process, and due to the harsh environment, molecules are broken up into fragments ${ }^{37}$. Different molecules fragment in distinct manners, and the way molecules are broken up, can be used for identification purposes ${ }^{37}$.

\subsubsection{Density Functional theory (DFT)}

Density functional theory (DFT) is a widely used ab initio quantum mechanical computational model to study molecular behavior and properties ${ }^{38}$. DFT is heavily based on evaluating the electronic structure of many molecules at atomic level, and construct a three-dimensional electronic density, and that the resulting electronic density function can be used as a basis to determine all molecular properties of the system of interest, such as molecular geometry and energy ${ }^{38}$. This approach proves to be extremely powerful 
in analyzing various chemical, biological and material systems, and that it helps shed light on mentioned systems beyond what experiments can provide ${ }^{38}$. The recent spike in the number of DFT-based publications in literature indicates the popularity of the method, as well as the increased confidence in its accuracy and reliability ${ }^{39}$. In spectroscopy, the development of time-dependent density functional theory (TD-DFT) has facilitated the use of DFT to investigate the photophysical properties of molecules ${ }^{40}$. Understanding the photophysical properties of a molecule requires the incorporation of the timedependence nature of light ${ }^{40}$. Therefore, knowledge of the electronic density is not sufficient, and that understanding its time dependence is crucial to carry out a comprehensive DFT investigation. Fortunately, the development of Runge-Gross theorem has shown that there is a unique mapping between time-dependent external potential of a system, and its time-dependent density, thus providing a roadmap to quantify the timedependent electronic density function of a system ${ }^{40}$. Once the time-dependent electronic density function of a system is obtained, all photophysical properties of the system of interest can be determined. Generally, TD-DFT is used to predict the absorption and emission spectra, as well as molecular geometries in both ground and excited states ${ }^{39}$. 

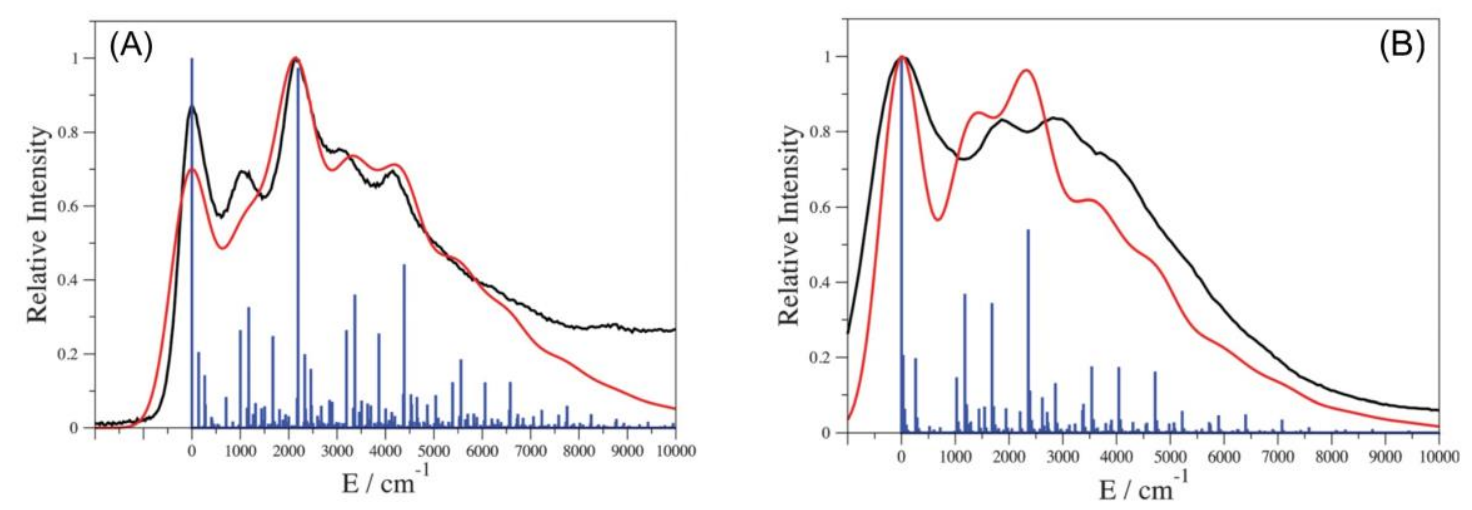

Figure 21. (A) Comparison of absorption spectrum of diphenylacetylene between experiment (black) and DFT-based calculation (red), readapted from ref..$^{39}$ (B) Comparison of emission spectrum of diphenylacetylene between experiment (black) and DFT-based calculation (red), readapted from ref. ${ }^{39}$

For example, diphenylacetylene's absorption and emission spectra have been obtained using DFT methodology, and compared with experimental results, see Figure 21. DFT calculations are able to capture all relevant spectrum properties: signal intensity and location. On the other hand, although peak locations were slightly off, DFT was able to predict the major peaks in the emission spectrum of diphenylacetylene, and overall shape of the graph. This shows that DFT can capture the overall spectral behavior of molecules with confidence. Moreover, DFT can be utilized to identify any molecular geometry changes upon excitation. Examining the most intense absorption peak at $2200 \mathrm{~cm}^{-1}$, one can rely on DFT to show that the lengths of $\mathrm{C}-\mathrm{C}$ bond in the acetylene functional group have been changed upon excitation, see Figure $22(A)$ and (B). Moreover, DFT can be utilized to identify any torsional motion that takes place upon excitation. Figure 22 (C) and (D) show that DFT is able to identify how 4-dialkylamino-2-benzylidene malonic acid dimethyl ester goes through a torsional motion upon excitation ${ }^{29}$. This shows the power of DFT calculations to track all molecular changes that take place once a molecule is 
excited. Since most spectroscopic experiments are carried out in solution, it is critical that the solvent effect is taken into account when performing DFT computations. Generally, there are two main schemes that can be utilized to incorporate the effect of the solvent: explicit and implicit models ${ }^{39}$. In the former, all solvent molecules are explicitly represented in the calculations ${ }^{39}$. However, in the latter, solvent is treated as a continuum that has the same macroscopic properties of the solvent of interest ${ }^{39}$. One of the most popular implicit models is the polarizable continuum model $(\mathrm{PCM})^{39}$. Although implicit solvent models fail to consider solute-solvent interactions, they allow estimations of media effects with relatively faster time and cheaper costs, while maintaining the desired level of accuracy ${ }^{39}$. Overall, DFT calculations are extremely powerful in enhancing our understanding of the photophysical properties of molecules, and the recent spike in the popularity of DFT-based studies hints on its effectivity. 

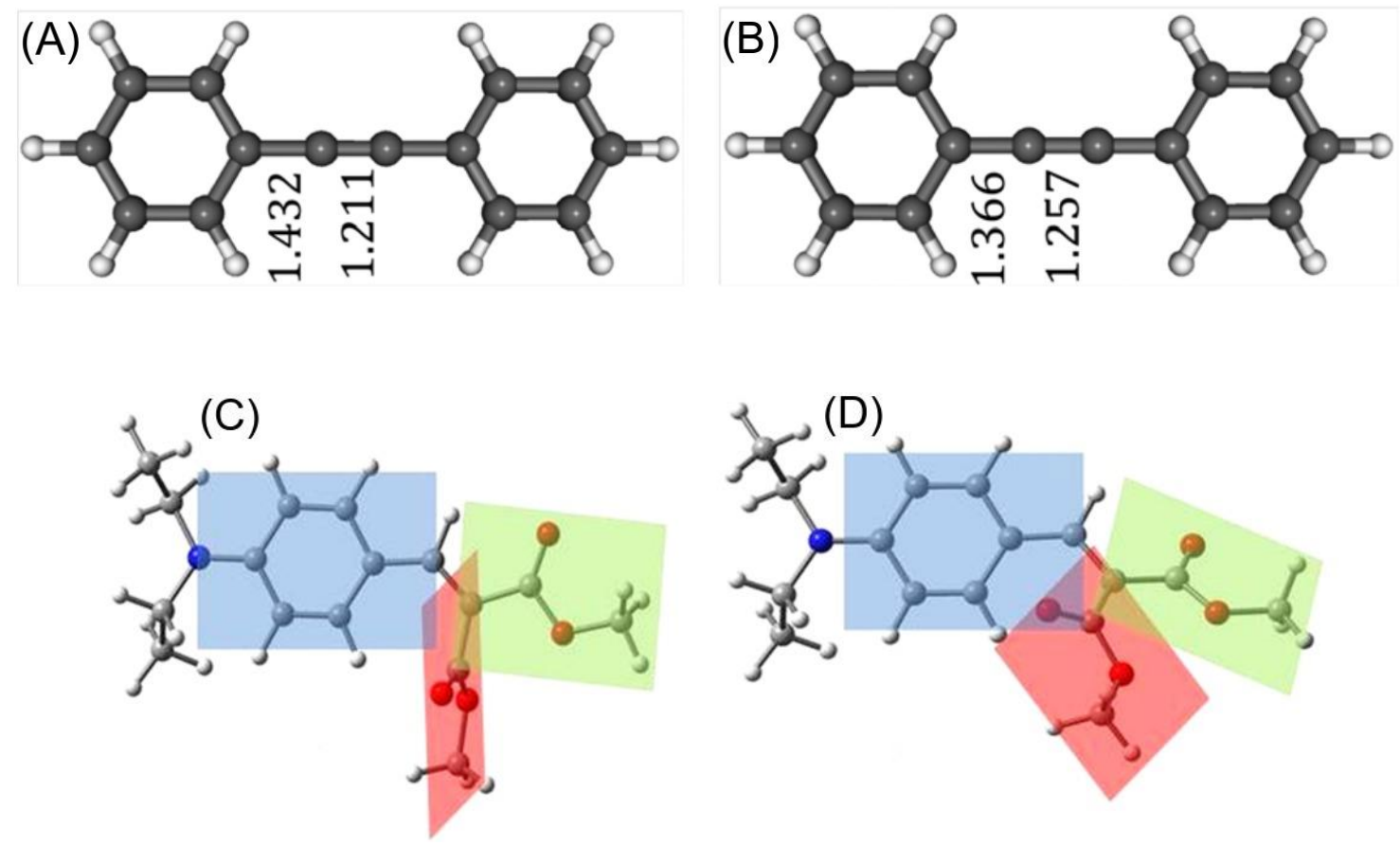

Figure 22. (A) DFT-optimized geometry of dipehnylacetylene in the ground state, readapted from ref..$^{39}$ (B) DFT-optimized geometry of dipehnylacetylene in the excited state, readapted from ref. ${ }^{39}$ (C) DFT-optimized geometry of 4-dialkylamino-2-benzylidene malonic acid dimethyl ester in the ground state, readapted from ref. ${ }^{29}$ (D) DFT-optimized geometry of 4-dialkylamino-2-benzylidene malonic acid dimethyl ester in the excited state, readapted from ref. ${ }^{29}$ 


\section{Chapter 2}

\section{Spectroscopic Analysis of the Influence of Twisting Motion of a BTDB Molecule on its Photophysical Properties}

\subsection{Introduction}

As mentioned in chapter 1 , organic compounds are used in a wide range of applications, and their fundamental photophysical properties, such as charge transfer and charge recombination, have been studied in several chemical systems the literature ${ }^{41}$. Moreover, given that several spectroscopic measurements are frequently carried out in solution, it is well-established that a highly concentrated solution can lead to erroneous results of the excited state dynamical processes of the sample of interest due to the formation of aggregates, and dimers of the solute $e^{42-43}$. Aggregates and dimers can have distinct spectroscopic properties from the monomer of the same molecule due to differences in structure rigidity, as an example ${ }^{42}$. To induce aggregation or dimerization, studies have shown that it is sufficient to prepare solutions in the milli-molar range ${ }^{44-51}$. Therefore, to help reveal the true identity of a single molecule of the sample of interest, highly concentrated solutions are usually avoided, and it is generally assumed that singlemolecule photophysical properties are revealed when reaching the micro-molar regime. Seeing that dimers and oligomers can have completely different spectral behavior from its monomer species, one can explain the differences via an examination of the molecular shape and rigidity. As mentioned in chapter 1 , structure rigidity plays a key role in controlling molecular photophysical properties, and can be used to tune them to be 
utilized in practical applications in science and engineering. In this chapter, it is shown that dimerization and oligomerization at low concentration, nano- and micro-molar, can influence structure rigidity of a molecule and the spectroscopic behavior of the molecule. Torsional motions are commonly known as a very efficient non-radiative deactivation mechanisms for organic molecules upon photon-excitation, and that dimerization and oligomerization can hinder torsional motions. ${ }^{52-60}$ Taken as a case in point, the Benzothiazole dicarboxylic derivative (BTDB) organic molecule has a rod-like structure with a carboxylic acid group at each end, see Figure 23 for the full chemical structure. BTDB molecule is used as a linker in MOF synthesis ${ }^{51,61}$ and as a fluorescent probe in biological applications due to its high $\pi$-conjugation, molar extinction coefficient, and high photostability. ${ }^{62}$ When preparing solutions of BTDB in solution at various concentrations in the nano- and micro-molar scale, distinct species arise through hydrogen-bond formation between carboxylic acid groups of different BTDB molecules. The mentioned species include dimers along with oligomers, chains of a small number of monomers linked, with a high formation constant of each. The formation of the mentioned species is fully supported by experimental measurements, as well as theoretical calculations. BTDB solutions at various concentrations give rise to distinct photophysical behavior in various spectroscopic measurements that include steady-state absorption and emission, photoluminescence quantum yield, lifetime, and NMR measurements. Furthermore, density functional theory (DFT) calculations show similar trends as found experimentally, confirming the proposed scheme of hydrogen-bond formation of BTDB molecules in the nano- and micro-molar scale. Examining the core causes of the distinct photophysical 
behaviors of the formed species of BTDB, one can utilize DFT calculations to probe molecular changes when hydrogen bonds are formed through an increase in concentration. It is shown that photophysical behavior of BTDB is heavily centered on the torsional motion of its aromatic groups around each other controlled by the formation of long-range hydrogen bonds between various monomers, and that the torsional motion is pathway is hindered upon dimerization and oligomerization.

To significantly suppress such non-radiative twisting motions and subsequently enhance the fluorescence quantum yields of such organic molecules, several chemical and material approaches have been established and developed, such as forming aggregation/suspensions at relatively high concentration in the presence of solvent mixtures, producing solid-state forms, constructing thin-films, incorporating heavy metal atoms, and embedding them in polymer chains. ${ }^{63-66}$ Nevertheless, these new approaches can induce other nonradiative processes, such as energy transfer, charge transfer, and defect states, that lead to different deactivation pathways of the excited state of the targeted molecules. ${ }^{42-50,55,67-73}$ However, this study reveals simple and novel approaches to obtain high PLQY and tunable emission spectral ranges in the liquid phase by optimizing the structural design of organic molecules. 


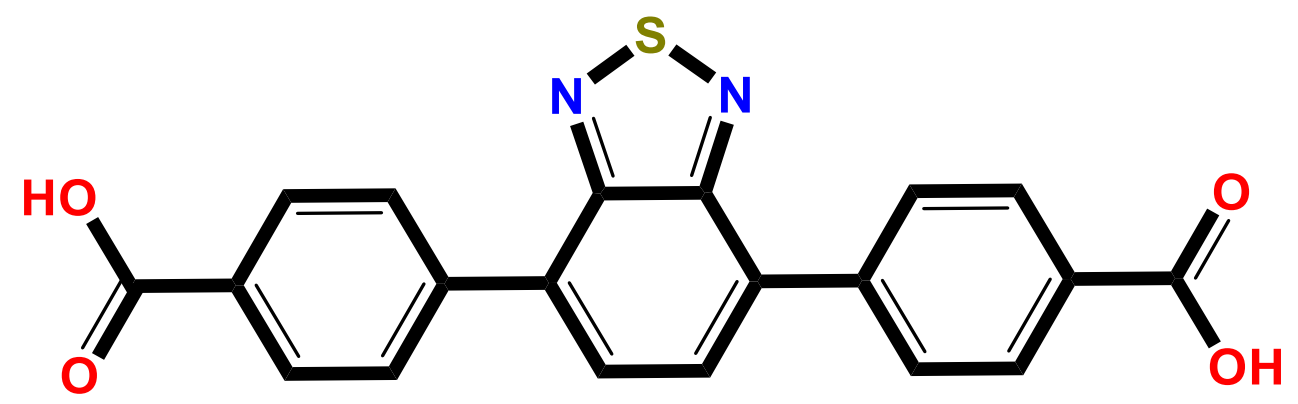

Figure 23. The chemical structure of the Benzothiazole dicarboxylic derivative (BTDB) organic molecule.

\subsection{Results and Discussion}

\subsubsection{Steady State Absorption and Emission}

Steady state absorption spectra have been obtained of BTDB linker at various concentrations in the $3-1000 \mu \mathrm{M}$ range in DMF. Upon increasing the concentration from $3 \mu \mathrm{M}$ to $1000 \mu \mathrm{M}$, a blue-shift of $12 \mathrm{~nm}$ is observed in the $S_{1}$ absorption band; $3 \mu \mathrm{M}$ has an absorption maximum centered at $390 \mathrm{~nm}$ while $1000 \mu \mathrm{M}$ has its maximum at $378 \mathrm{~nm}$, see Figure 24 (A) for details. On the other hand, emission spectra of the corresponding solutions show dramatic changes upon increasing the concentration in the 0.1-64 $\mu \mathrm{M}$ range in DMF. At $0.1 \mu \mathrm{M}$, a broad emission peak is centered at $485 \mathrm{~nm}$. Upon increasing the concentration, a systematic increase in intensity and broadness of the emission peak is observed before it splits at $3 \mu \mathrm{M}$ to form two distinct peaks. Upon increasing the concentration away from $3 \mu \mathrm{M}$, the separation between the two peaks becomes more significant. At $64 \mu \mathrm{M}$, the two distinct peaks are centered at $420 \mathrm{~nm}$ and $620 \mathrm{~nm}$. Figure 24 (B) shows the dynamical change of the emission behavior of BTDB linker in DMF at various concentrations. 

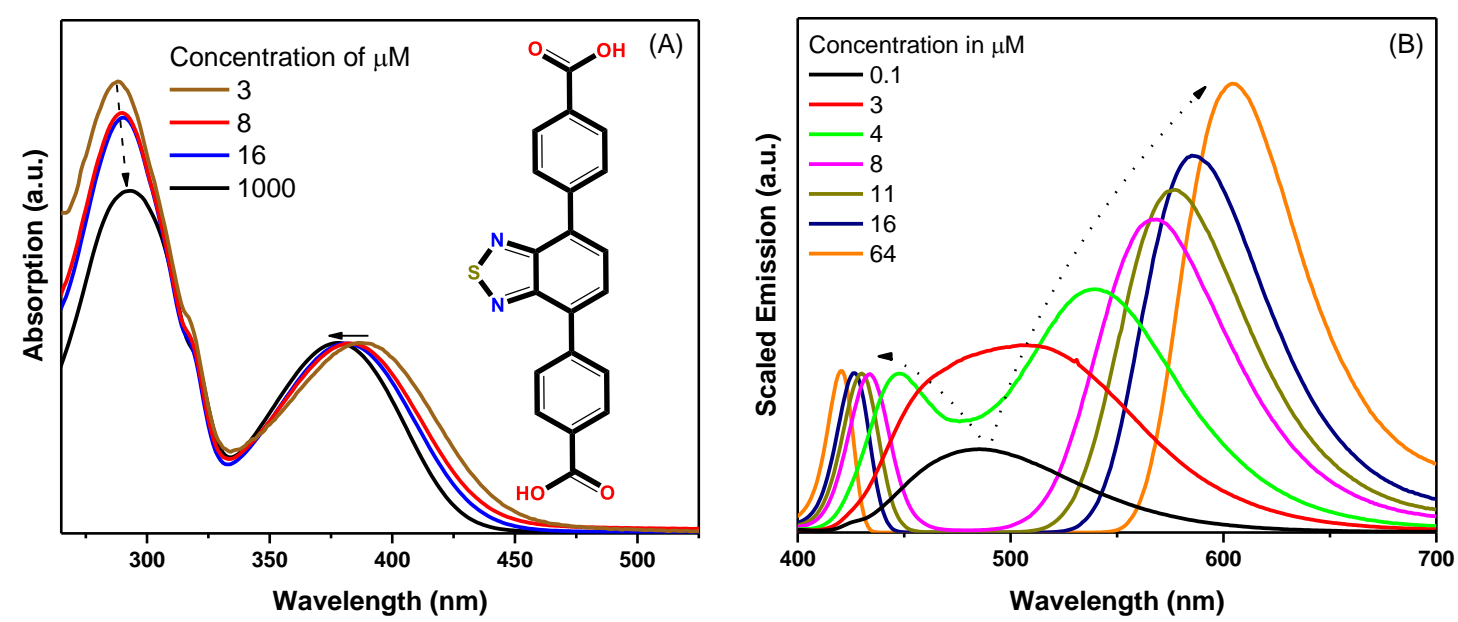

Figure 24. (A) Steady state absorption spectra of BTDB molecules in DMF at various concentrations in the range of 3-1000 $\mu \mathrm{M}$. (B) Emission spectra of BTDB molecules in DMF at various concentrations in the range of 0.1-64 $\mu \mathrm{M}$.

Upon increasing the concentration of BTDB in DMF beyond $64 \mu \mathrm{M}$, emission spectra evolve in a way that the two distinct peaks approach each other, and eventually merge to form one broad peak centered at $480 \mathrm{~nm}$, when reaching $1000 \mu \mathrm{M}$, see Figure 25 for details.

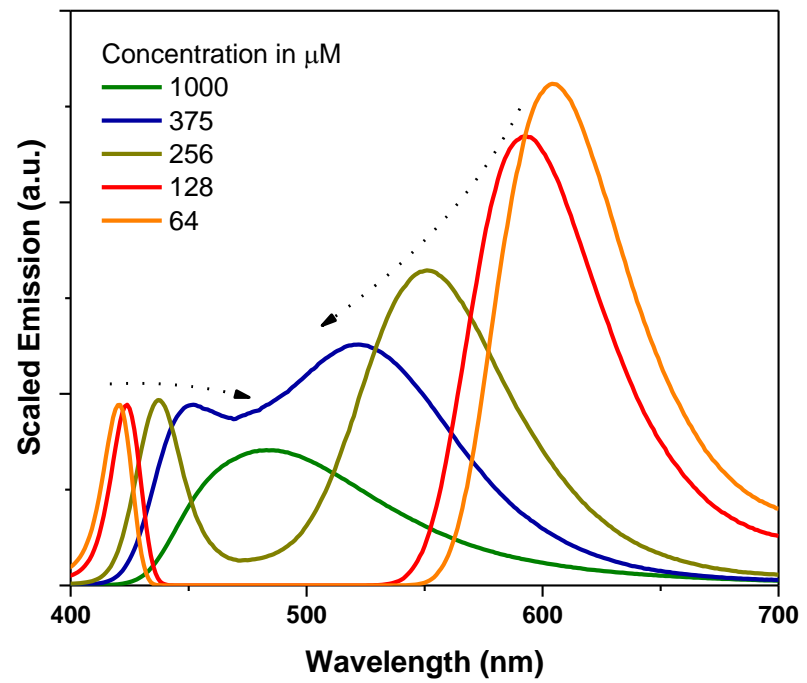

Figure 25. Emission spectra of BTDB molecule in DMF in the concentration range of 64$1000 \mu \mathrm{M}$. 
To rule out the contribution of solvent molecules to the observed spectral changes with concentration, spectral measurements have been carried out of various concentrations of BTDB in solvents of different properties such as dielectric constant and dipole moment (see Table 3), and that similar emission trends have been observed, confirming that the contribution of the solvent is negligible, see Figure 26 for details ${ }^{74}$. Solvent selection was heavily based on BTDB solubility, along with maximizing the variance in dielectric constant and dipole moment of selected solvents.
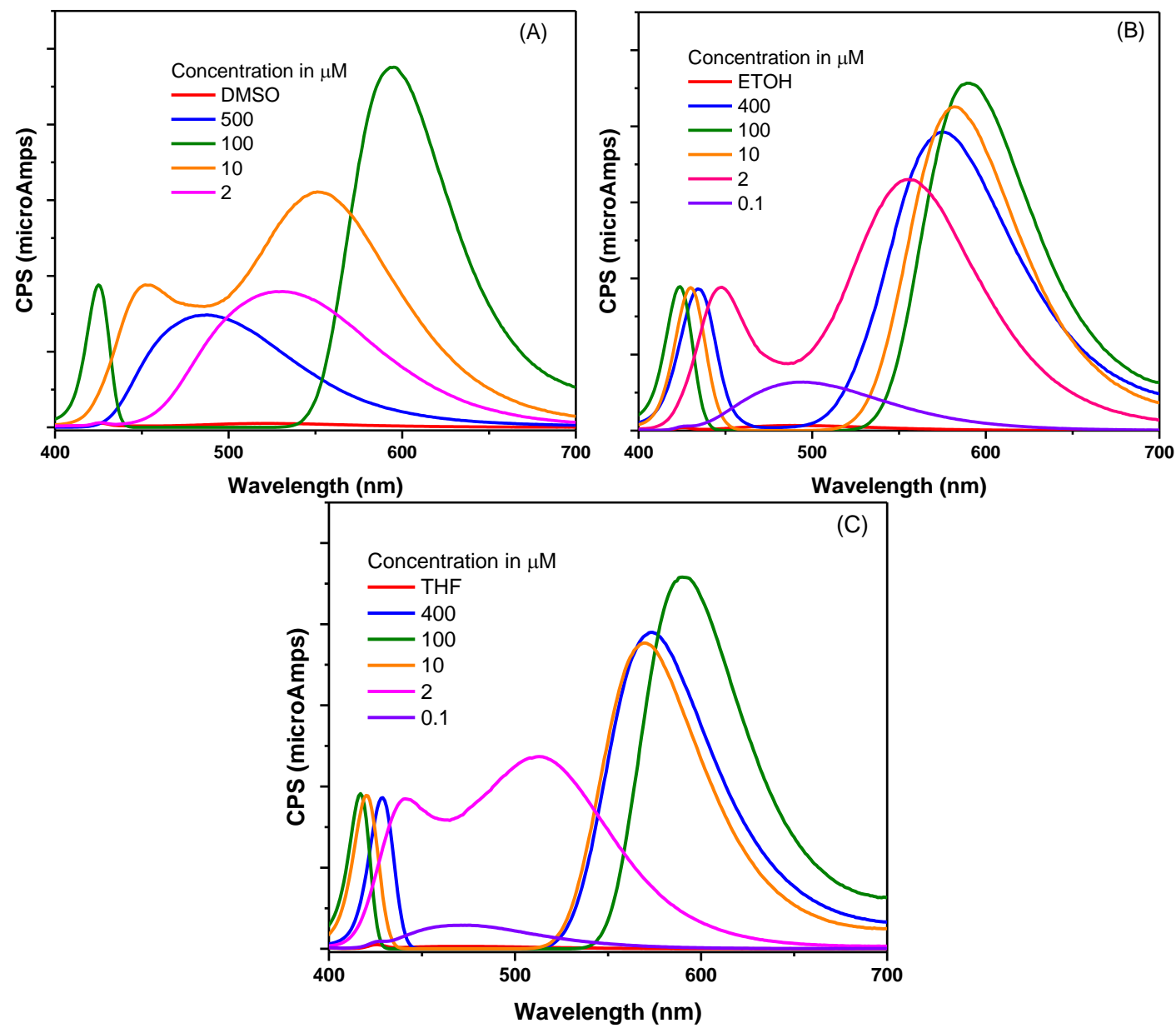

Figure 26. (A) Emission spectra of BTDB molecule at various concentrations in DMSO. (B) Emission spectra of BTDB molecule at various concentrations in EtOH. (C) Emission spectra of BTDB molecule at various concentrations in THF. 
Table 3. Dielectric constant and dipole moment numerical values of DMF, DMSO, EtOH, and THF

\begin{tabular}{|c|c|c|}
\hline Solvent & Dielectric Constant & Dipole Moment $^{74}$ (D) \\
\hline DMF & 38 & 3.82 \\
\hline DMSO & 46.7 & 3.92 \\
\hline ETOH & 24.5 & 1.69 \\
\hline THF & 7.5 & 1.75 \\
\hline
\end{tabular}

The observed spectral changes with concentration suggest that distinct species are formed upon increasing the concentration of BTDB. Given that BTDB and solvent molecules are the only species present in prepared samples, one may hypothesize the existence of either an aggregation equilibrium due to $\pi-\pi$ interaction or dimerization equilibrium due to hydrogen-bond formation.

\subsubsection{Density Functional Theory (DFT) Calculations}

To shed more light on the existing ongoing mechanism, DFT calculations have been performed to establish the equilibrium process responsible for the described spectral changes. DFT results show that there are two excited states of BTDB in DMF at 412 and $312 \mathrm{~nm}$, see Figure 27 (A). Examining the lowest excited state transition, $\mathrm{S}_{1}$ and known as HOMO-LUMO transition, one observes that the optimized dihedral angle is ca. $37.5^{\circ}$ around the benzothiazole moiety in the ground state, while changes to ca. $18.2^{\circ}$ upon excitation, see Figure 27 for details. This change in the dihedral angle, along with the calculated Stock's shift of ca. $5580 \mathrm{~cm}^{-1}$, highlights the role of torsional processes such 
as isomerization and twisting motions in relaxing/deactivating the excited state of BTDB, as shown in other organic dyes in the literature $53,55-56,58,75-76$.
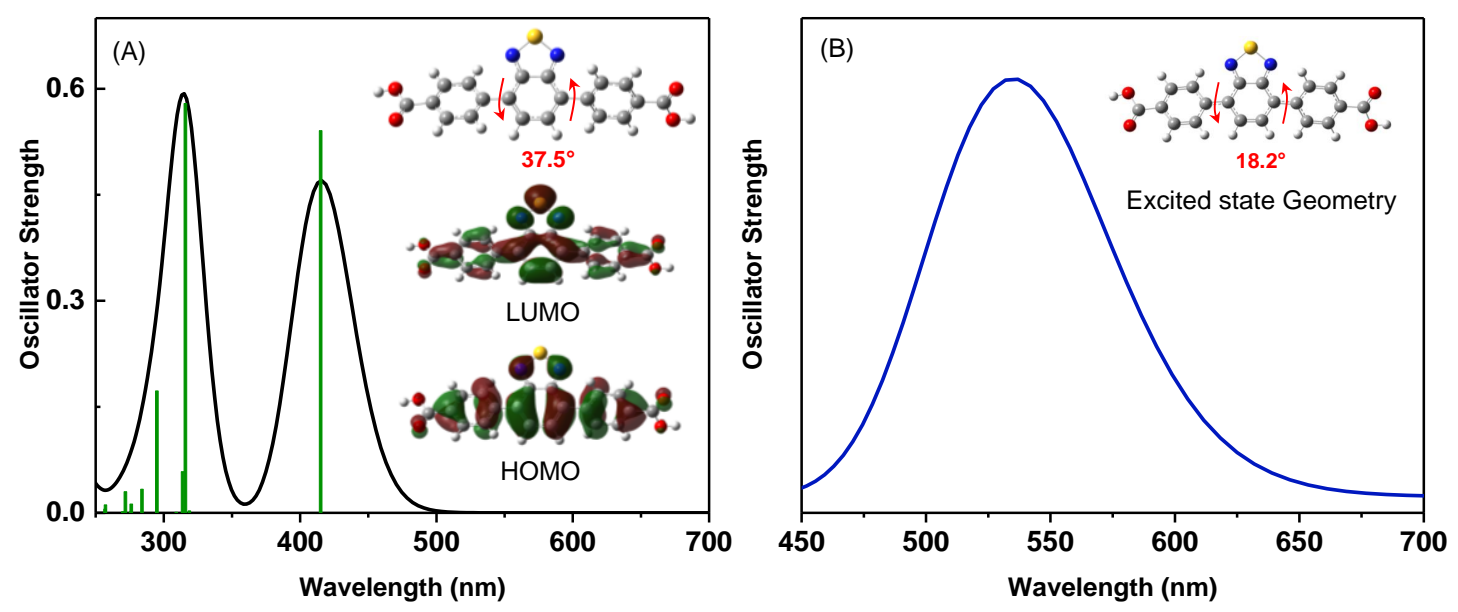

Figure 27. (A) Calculated absorption spectrum of BTDB in DMF, along with the optimized ground state structure and the dihedral angle, while showing the electronic density of HOMO and LUMO states. (B) Calculated emission spectrum of BTDB in DMF, along with the optimized excited state structure and the dihedral angle.

Furthermore, DFT calculations have been performed to establish the dependence of emission maximum of BTDB molecule on the dihedral angle around the benzothiazole moiety. Figure 28 shows that larger dihedral angles from optimized geometry result in blue-shifted emission spectra, thus highlighting the correlation between the emission maxima and the thermalized dihedral angle. 


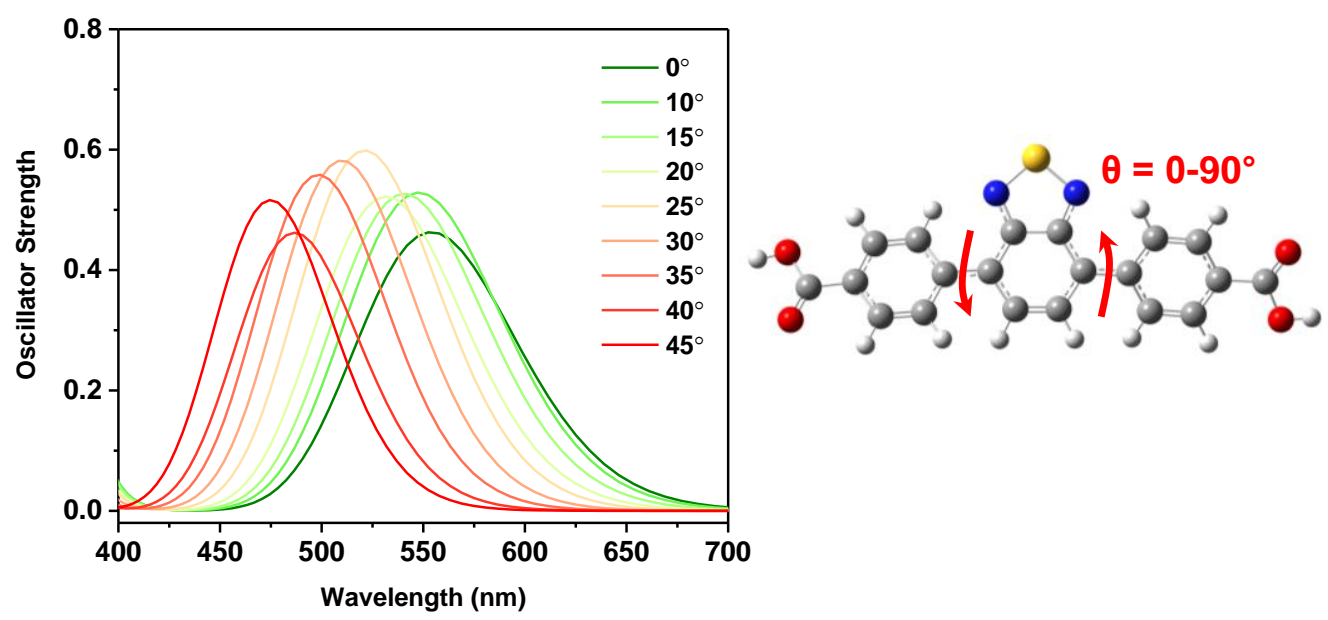

Figure 28. Calculated emission spectra of BTDB molecule in DMF with various dihedral angle between phenyl units.

To help determine the type of equilibrium responsible for the spectral change with concentration, two possible structures of BTDB were optimized through DFT methodologies. The first proposed structure features two BTDB molecules aggregated on top of each other due to $\pi-\pi$ stacking, while the second structure features a linear dimer structure of BTDB through hydrogen-bond formation, see Figure 29 for details. Figure 29 shows that DFT calculations predict that the aggregate would have a blue-shifted emission spectra of about ca. $60 \mathrm{~nm}$ with respect to the monomer. However, a single emission peak does not correspond to experimental results, thus suggesting it is unlikely that $\pi-\pi$ stacked aggregates exist. On the other hand, DFT calculations predict that the dimer would have two distinct emission peaks, and that the calculated emission behavior corresponds to the experimental results. Moreover, theoretical calculations shed light on how two emission peaks arise in the case of the dimer species. Since a dimer would have two monomers that they correspond to different dihedral angles, it is expected that the dimer would generate two distinct emission peaks since it is established that emission 
maximum of BTDB molecule is heavily influenced by the angle. Therefore, one can conclude that upon increasing the concentration of BTDB in DMF to $64 \mu \mathrm{M}$, two monomers come together to form a dimer through hydrogen bonding interaction, facilitated by the presence of a carboxylic acid group of each end. Interestingly, the dihedral angle in the dimer is different from the one in the monomer, indicating that differences in excited state lifetime and photoluminescence quantum yield will be observed, as shown in a later section.
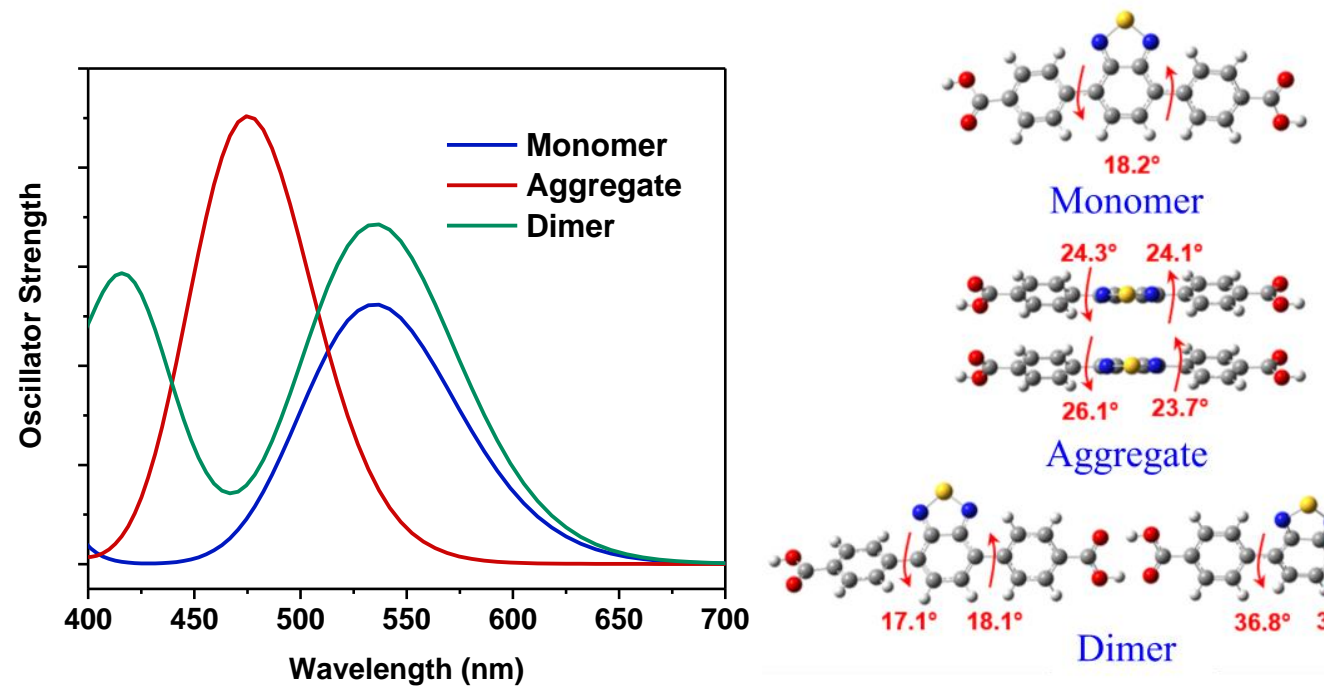

Aggregate

Figure 29. Calculated emission spectra of monomer BTDB molecule, aggregate of two $B T D B$ molecules through $\pi-\pi$ stacking, and dimer of BTDB molecules through hydrogenbond formation, along with optimized structure of each species and dihedral angles.

On the other hand, analyzing the second equilibria when concentration is increased from $64 \mu \mathrm{M}$ to $1000 \mu \mathrm{M}$, one can postulate that hydrogen-bond formation is the main mechanism driving the spectral change, and that dimers tend to bind together to form a chain of few number of monomers, namely as an "oligomer". In other words, increasing the concentration away from $64 \mu \mathrm{M}$, dimers tend to bind together through 
their carboxylic acid ends via hydrogen-bond formation, to make a linear oligomer. To test the oligomer hypothesis, DFT calculations were performed to project emission spectra of few number of BTDB-based linear oligomer. Figure 30 shows that upon increasing the number of the monomers of an oligomer, a significant spectral change takes place. Starting from a two-peak emission spectrum at the dimer case, extending the length of the oligomer seems to systematically enhance the intensity of one emission peak, while reducing the intensity of the other. Therefore, one may hypothesize the calculated systematic trend in emission spectrum will continue as the length of the oligomer is extended even further than four monomers. As a result, upon increasing the length of the oligomer species, the observed two emission peaks will collapse to lead to the formation of a single emission peak. This insight is in full agreement with the experimental observations that upon increasing the concentration from $64 \mu \mathrm{M}$ to 1000 $\mu \mathrm{M}$, the observed two-emission-peak spectrum will collapse to lead into a one-emissionpeak spectrum. Experimental evidence will be provided in later sections to support the proposed mechanisms. 

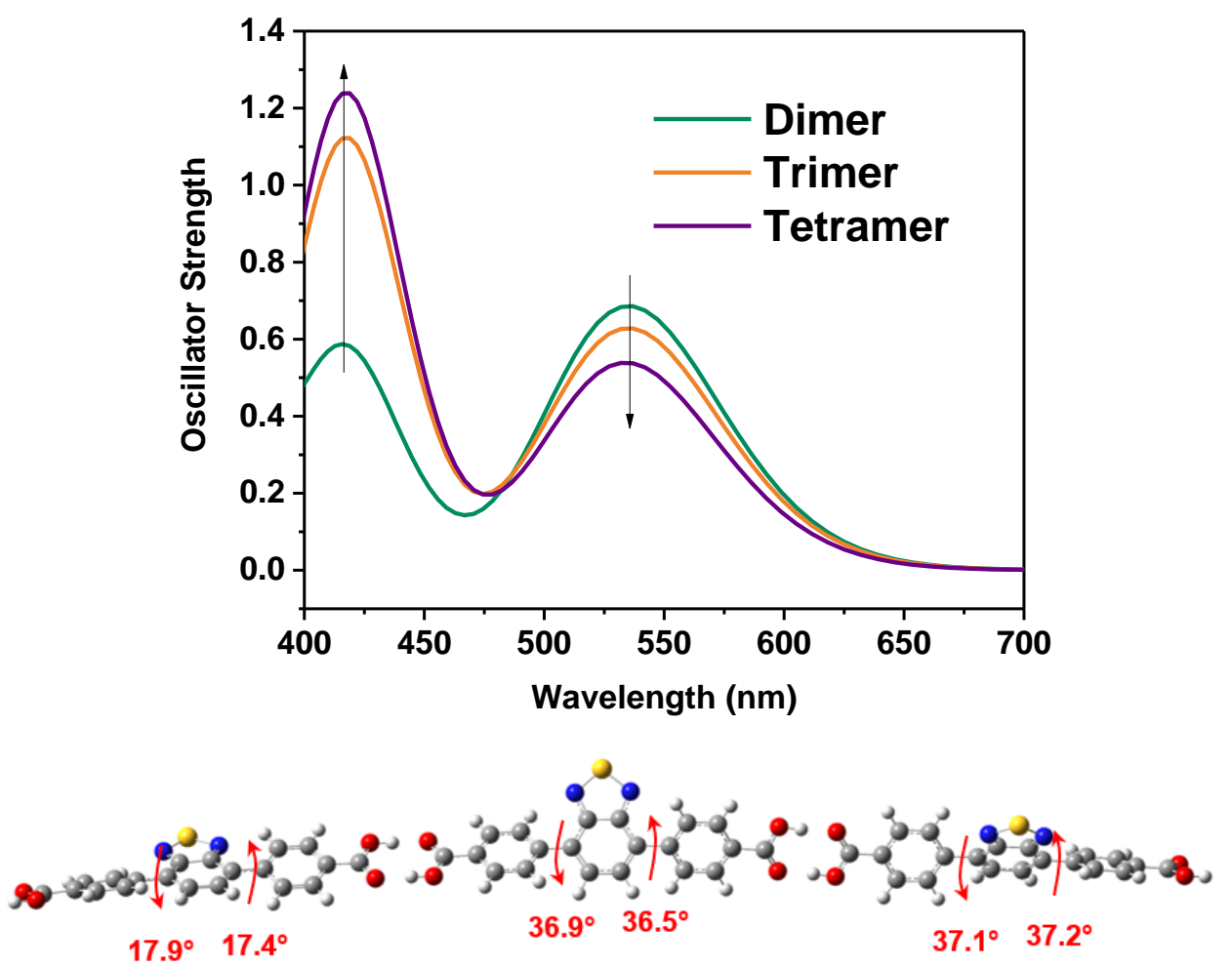

Figure 30. Calculated emission spectra of several BTDB-based linear oligomers: dimer, trimer, and tetramer.

\subsubsection{Equilibrium Calculations}

Postulating that increasing the concentration of BTDB in DMF in the range of 0.1$64 \mu \mathrm{M}$, a dimer formation equilibrium is dominating, and influencing the observed spectral change, one can derive an equation relating the observed emission intensity of a sample and the concentration of the linker at a specific wavelength. Previous results suggest that the following equation holds:

$$
M+M \stackrel{K_{e q}^{D}}{\longleftrightarrow} D
$$


Where $M$ represents the monomer species, $D$ represents the dimer species, and $K_{e q}^{D}$ represents the dimer formation equilibrium constant.

The monomer-dimer equilibrium constant $K_{e q}^{D}$, can be written as follows:

$$
K_{e q}^{D}=\frac{[D]}{[M]^{2}}
$$

The total concentration of BTDB in solution, $C_{0}$, can be expressed as follows:

$$
C_{0}=[M]+2[D]
$$

The total emission intensity, $I_{e m}^{\text {total }}$, is the total sum of the emission of the monomer $\left(I_{e m}^{M}\right)$ and that of the dimer $\left(I_{e m}^{D}\right)$ normalized to their fractional concentrations in solution, and is expressed as follows:

$$
I_{e m}^{\text {total }}=I_{e m}^{M} \frac{[M]}{C_{0}}+I_{e m}^{D} \frac{2[D]}{C_{0}}
$$

When solving the system of equations, equations 1,2 , and 3 together, the following expression can be obtained:

$$
I_{e m}^{\text {total }}=\left(I_{e m}^{M}-I_{e m}^{D}\right) \frac{2}{\left(\sqrt{8 K_{e q} C_{0}+1}+1\right)}+I_{e m}^{D}
$$

Using nonlinear least squares fitting method and equation 4, one can plot the total emission intensity at $680 \mathrm{~nm}$ at various concentrations, see Figure 31 for details. Moreover, using fitting data, the dimer formation equilibrium constant can be estimated to be $18,000 \mathrm{M}^{-1}$. The high numerical value of the equilibrium constant is in full agreement 
with the experimental observation of forming observable amounts of dimer of BTDB molecules in the nano- and micro- molar scale.

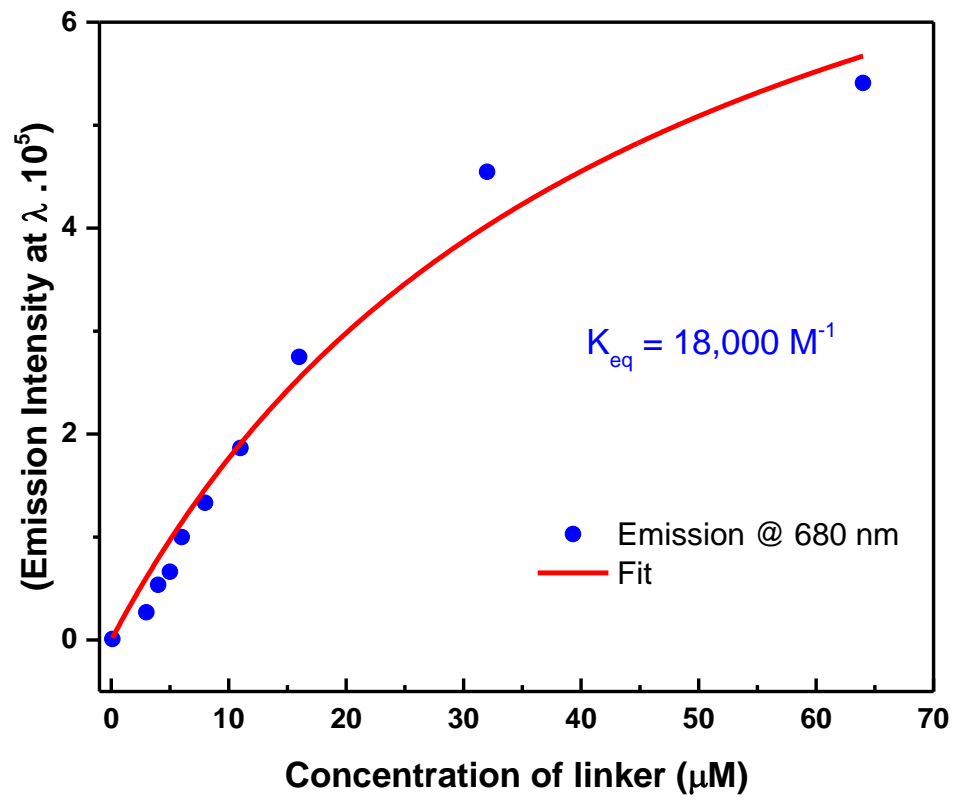

Figure 31. A plot of the total emission intensity with respect to BTDB concentration, along with a nonlinear least squares fitting model based on equation 4.

Upon increasing the concentration of BTDB in DMF beyond $64 \mu \mathrm{M}$, emission spectra evolve in a way that the two distinct peaks approach each other, and eventually merge to form one broad peak centered at $480 \mathrm{~nm}$, when reaching $1000 \mu \mathrm{M}$, see Figure 25 for details.

Postulating that hydrogen-bond formation is the main mechanism driving the spectral change as concentration is increased beyond $64 \mu \mathrm{M}$, and that dimers tend to approach each other to form longer linear chains, one can derive an equation relating the observed emission intensity of a sample and the concentration of the linker at a specific wavelength. Therefore, the following equation holds: 


$$
\begin{gathered}
76 \\
n D \stackrel{K_{e q}^{O}}{\longleftrightarrow} 0
\end{gathered}
$$

Where $n$ represents the number of dimers involved, $D$ represents the dimer species, $O$ represents the oligomer species, and $K_{e q}^{O}$ represents the oligomer formation equilibrium constant.

The dimer-oligomer equilibrium constant $K_{e q}^{O}$, can be written as follows:

$$
K_{e q}^{O}=\frac{[O]}{[D]^{n}}
$$

The total concentration of BTDB in solution, $C_{0}$, can be expressed as follows:

$$
C_{0}=[D]+n[O]
$$

The total emission intensity, $I_{e m}^{\text {total }}$, is the total sum of the emission of the monomer $\left(I_{e m}^{D}\right)$, as well as that of the dimer $\left(I_{e m}^{O}\right)$ normalized to their fractional concentrations in solution, and is expressed as follows:

$$
I_{e m}^{\text {total }}=I_{e m}^{D} \frac{[D]}{C_{0}}+I_{e m}^{O} \frac{n[o]}{C_{0}}
$$

When solving the system of equations, equations 5,6 , and 7 together, the following expression can be obtained:

$$
I_{e m}^{\text {total }}=\left(I_{e m}^{D}-I_{e m}^{O}\right) \frac{1}{\left(n K_{e q}\left[C_{0} /(n+1)\right]^{n-1}+1\right)}+I_{e m}^{O}
$$


While incorporating following assumption that $[D]=C_{0} /(n+1)$, in which the absorptivity is unchanged at this concentration regime (32 $\mu \mathrm{M}-1 \mathrm{mM})$, see Figure 32 for details.

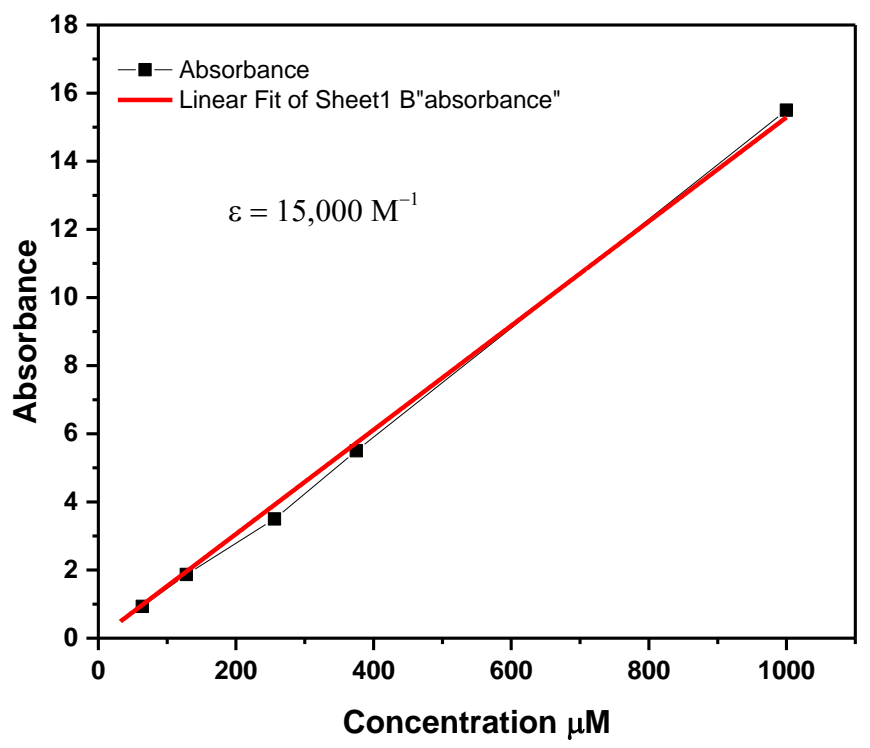

Figure 32. Beer's law for BTDB from 32-1000 $\mu \mathrm{M}$ in DMF.

Using nonlinear least squares fitting method and equation 8, one can plot the total emission intensity at $680 \mathrm{~nm}$ at various concentrations, see Figure 33. Equation 8's accuracy in modeling the experimental data to a high degree of accuracy, verifies the initial postulates that oligomer formation through hydrogen-bond formation in the 64$1000 \mu \mathrm{M}$ region. Other overwhelming evidence will be introduced later in the section. Therefore, using fitting data, the oligomer formation equilibrium constant can be estimated to be ca. $1.2 \times 10^{13} \mathrm{M}^{-3}$, and $\mathrm{n}$ to be ca. 4 . The high numerical value of the equilibrium constant is in full agreement with the experimental observation of forming oligomers of BTDB molecules in micro-molar scale. 


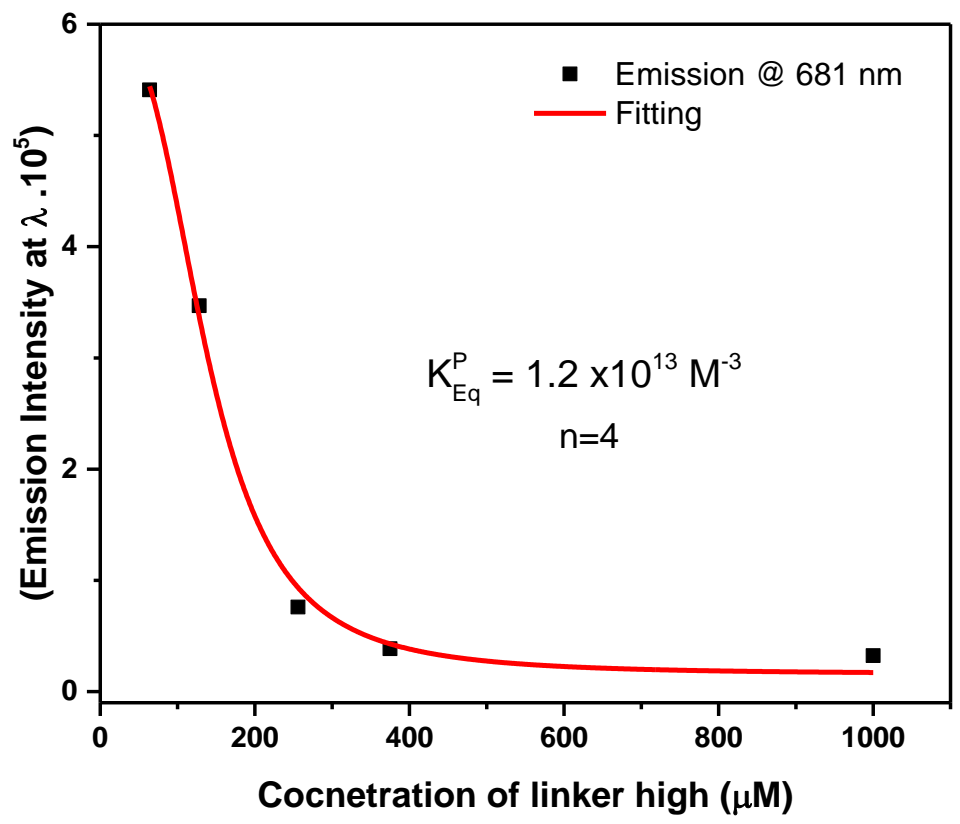

Figure 33. A plot of the total emission intensity with respect to BTDB concentration, along with a nonlinear least squares fitting model based on equation 8.

\subsubsection{H-NMR Measurements}

In addition, ${ }^{1} \mathrm{H}$ NMR measurements of BTDB at concentrations of 64 and $1000 \mu \mathrm{M}$ demonstrate the change in the electronic environment of the carboxylic acid hydrogen when concentration is increased. Upon increasing the BTDB concentration from 64 to $1000 \mu \mathrm{M}$, full width at half-maximum (FWHM) is increased from 0.084 PPM to $0.144 \mathrm{ppm}$ that is assigned to extensive hydrogen bonding interaction, see Figure 34. 


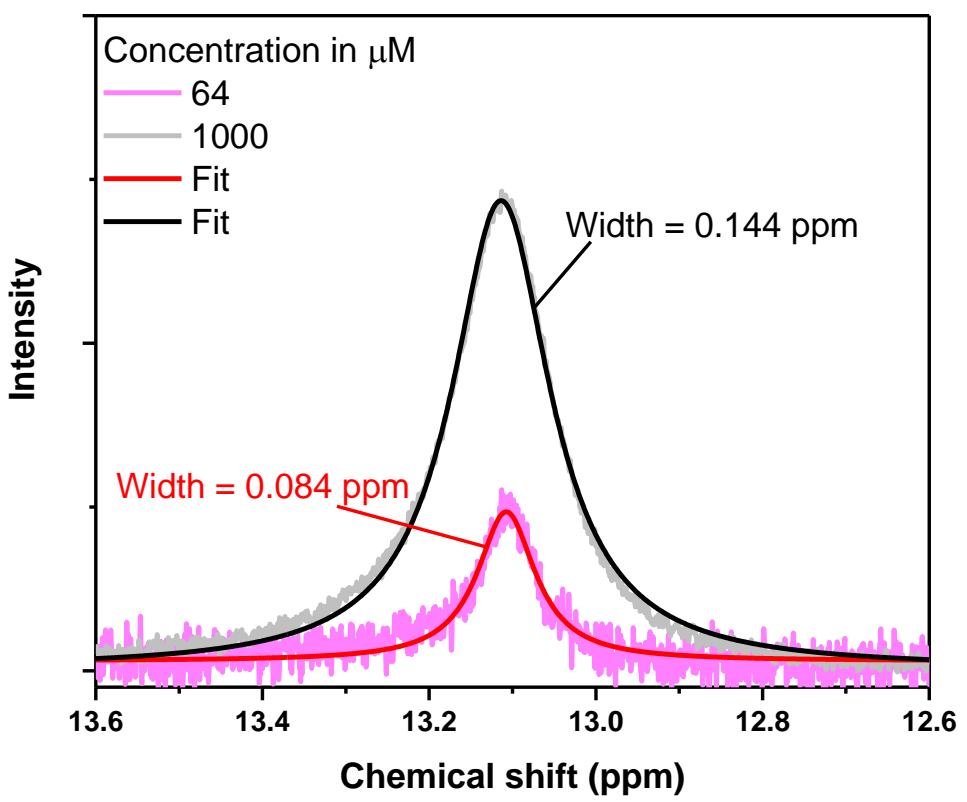

Figure 34. H-NMR zoomed-in on the carboxylic acid region to show how FWHM is positively correlated with concentration of $B T D B$, and indicating extensive hydrogen-bond formation when increasing the concentration.

\subsubsection{Deprotonation of Carbocyclic Acid Functionality}

The suggested scheme asserts that dimerization and oligomerization processes undergo in an extremely low concentration range, and that they are heavily facilitated by the fact that BTDB molecules have a rod-like shape with a carboxylic acid group on each end. The presence of two carboxylic acid groups per molecule facilitates the formation of dimers, as well as small soluble oligomers. Looking at the literature, one finds that dimerization of molecules containing carboxylic acid functionality has been well studied ${ }^{50}$, 68. However, all investigated molecules have only one carboxylic acid group per molecule, and have low values of equilibrium constant of formation ${ }^{49-50,68}$. Therefore, BTDB's ability to form dimers and oligomers at low concentrations with high equilibrium constants of formation, stems from the presence of two carboxylic acid groups per molecule. To 
further verify the role of hydrogen-bond formation in the observed spectral changes, a deprotonation test was carried out. In the test, an organic base, diethyl amine, was added to two different concentrations of BTDB, 64 and $1000 \mu \mathrm{M}$, which correspond to dimers and oligomers, respectively. Adding excess of the base should in principle deprotonate all carboxylic acid groups as follow:
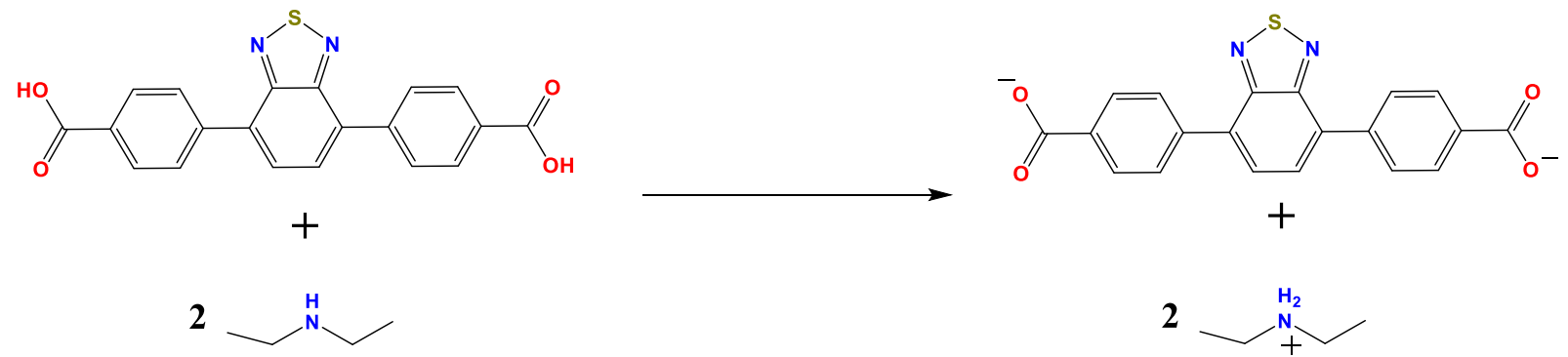

The equilibrium constant of the deprotonation reaction is estimated to be in the order of magnitude of $10^{6}$, and that adding excess of diethyl amine should deprotonate all carboxylic acid groups ${ }^{77}$. Figure 35 shows the absence of carboxylic acid peak disappeared on $\mathrm{H} \mathrm{H}-\mathrm{NMR}$ upon the addition of the organic base, thus confirming that all BTDB molecules have been deprotonated. 


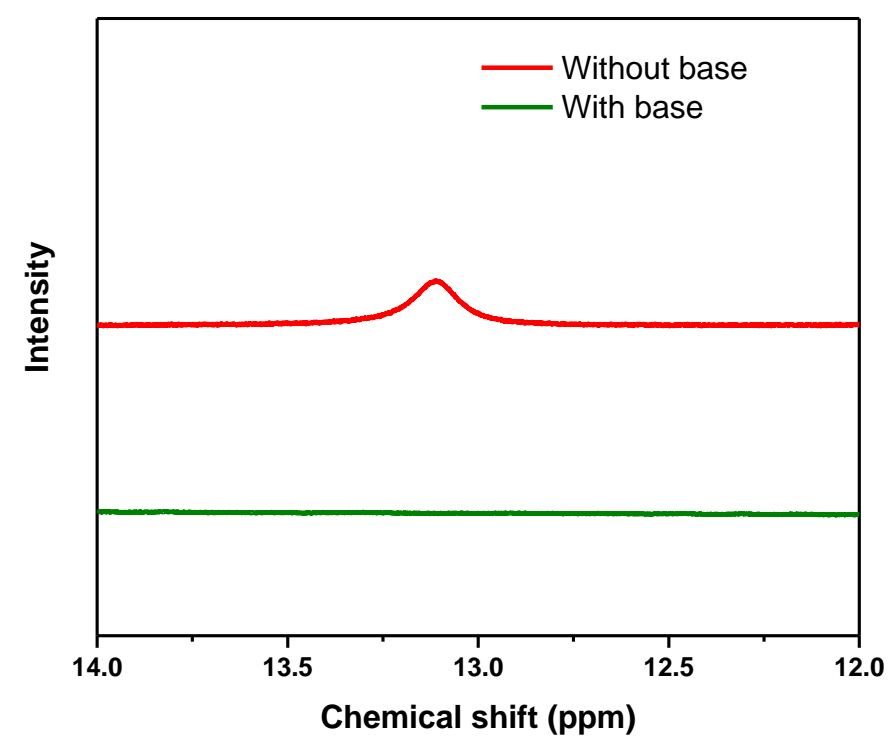

Figure 35. H-NMR spectra showing the disappearance of the carboxylic acid peak upon the addition of an organic base to a BTDB solution.

Deprotonating BTDB molecules eliminates the possibility of forming complex structures through hydrogen-bond formation, and that adding a base helps collapse all structures that resulted from dimerization and oligomerization processes. Therefore, it is expected that upon the addition of the base, no spectral change is observed with concentration, and that photophysical properties should correspond to the monomer species. Figure 36 shows that upon the addition of an organic base to 64 and $1000 \mu \mathrm{M}$ of BTDB in DMF, steady state absorption and emission of the two concentrations coincided, and that they started resembling the absorption and emission spectra of the monomer. Interestingly, in the case of $64 \mu \mathrm{M}$, adding an organic base facilitates the collapse of the two distinct emission peaks into one, thus resembling the emission spectra of the monomer. Therefore, the deprotonated test verifies that the proposed scheme that hydrogen-bond formation is indeed the underlying principle behind all spectral changes with concentration. Interestingly, there is a slight difference when comparing spectral 
data of the monomer species, and of the deprotonated species. The slight difference arises from the fact that monomer species are protonated, and that their carboxylic acid groups are intact, thus the electron density around the carboxylic acid groups would be slightly different from the deprotonated species.
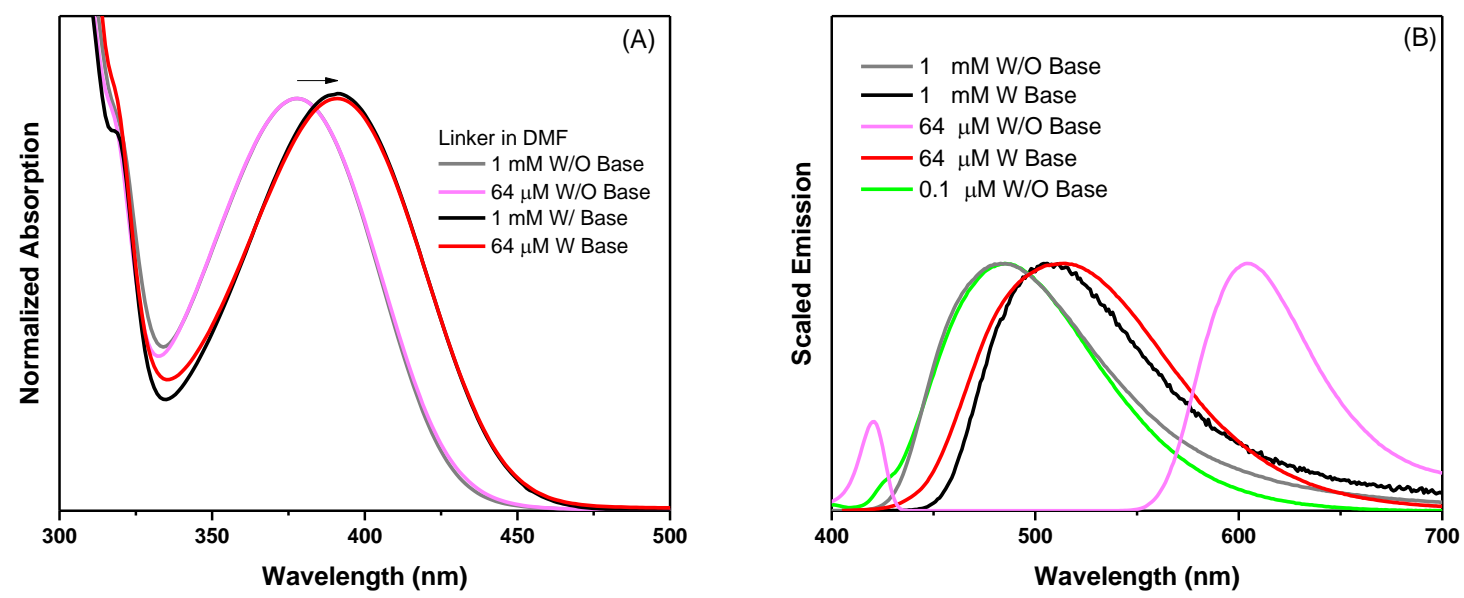

Figure 36. (A) Normalized absorption spectra of 64 and $1000 \mu \mathrm{M}$ of linker in DMF in the absence and presence of organic base. (B) Scaled emission spectra of 64 and $1000 \mu \mathrm{M}$ of linker in DMF in the absence and presence of organic base, while showing the similarity between deprotonated species and the monomer species, taken to be $0.1 \mu \mathrm{M}$.

\subsubsection{Photophysical Properties of Ester-form of BTDB Organic Molecule}

In addition, to further prove the influence of hydrogen-bond formation in the observed spectral behavior change, carboxylic acid functionality has been replaced with ester functionality, methyl ester functional groups. Due to the loss of the hydrogen atom of the carboxylic acid, the ester form of BTDB molecules are no longer able to sustain hydrogen-bond formation. Therefore, it is predicted that no change in spectral behavior will take place as concentration is increased. Figure 37 shows that one emission peak is observed across concentrations in the range $0.1-1000 \mu \mathrm{M}$, and that it is located at 475 
$\mathrm{nm}$. This experimental observation supports the proposed hypothesis again that hydrogen-bond formation is the driving force behind all spectral changes with concentration of BTDB molecules in solution; eliminating the possibility of hydrogen-bond formation removes all potential dynamic spectral changes with concentration.

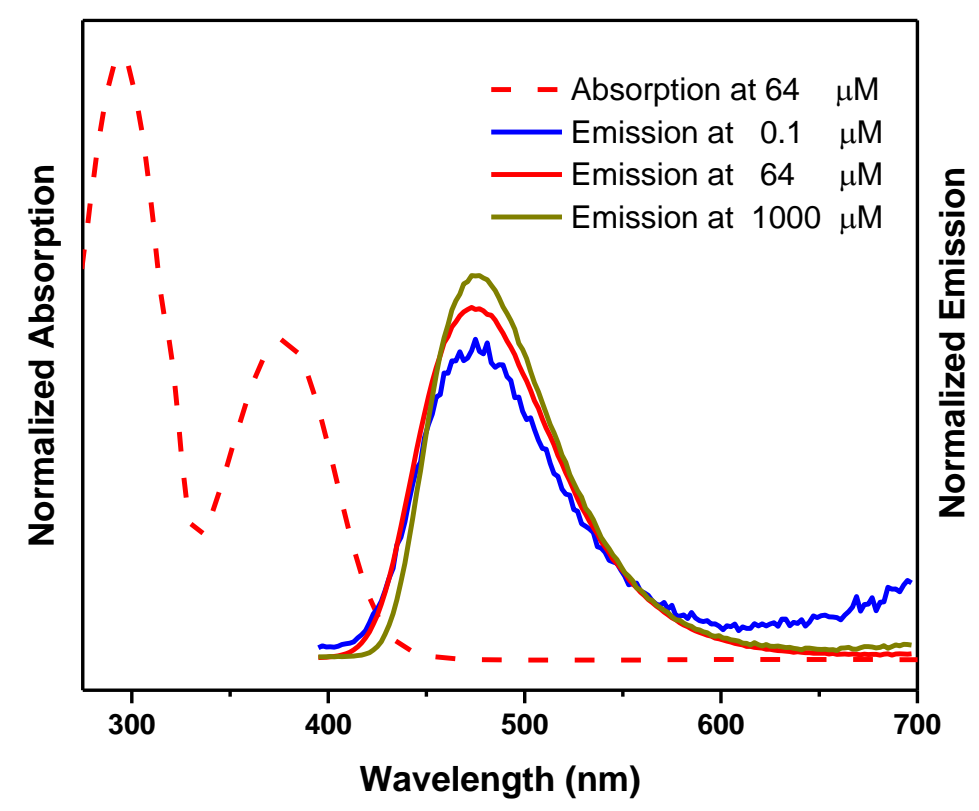

Figure 37. Normalized absorption and emission spectra of the ester-form of BTDB molecules, carboxylic acid functionality is replaced with ethyl ester, across three different concentrations.

\subsubsection{Excited State Lifetime and Emission Quantum Yield Measurements}

The BTDB species have different excited state dynamics at various concentrations.

Figure 38 demonstrates that time-resolved photoluminescence (TRPL) kinetics varies significantly with concentration of BTDB linker. Taken $0.1 \mu \mathrm{M}$ to represent monomer species, $64 \mu \mathrm{M}$ to represent dimer species, and $1000 \mu \mathrm{M}$ to represent oligomer species, all kinetics are fitted with global fitting procedure using exponential decay equations: 
$A_{1} e^{-t / \tau 1}+A_{2} e^{-t / \tau 2}$. The oligomer species at $1000 \mu \mathrm{M}$ can be modeled using a single exponential decay of $8.3 \mathrm{~ns}$, corresponding to the presence of the oligomer species. When decreasing the concentration to $64 \mu \mathrm{M}$, emission decays at $420 \mathrm{~nm}$ and $660 \mathrm{~nm}$ are similar, and give rise to a primary exponential decay of ca. $5 \mathrm{~ns}$ and a secondary exponential decay of $8.3 \mathrm{~ns}$, indicating the presence of a trace amount of oligomer species, see Table 4 for details. On the other hand, examining the $0.1 \mu \mathrm{M}$ sample, a fast excited state decay is recorded of $0.95 \mathrm{~nm}$, see Table 4. Moreover, emission decay of 1000 $\mu \mathrm{M}$ upon the addition of an organic base becomes faster, and begins to resemble the emission decay of the $0.1 \mu \mathrm{M}$ sample. Therefore, this observation further confirms the proposed scheme that hydrogen-bond formation is the underlying principle behind all spectral changes, and that deprotonating linkers induce them to behave as monomers.

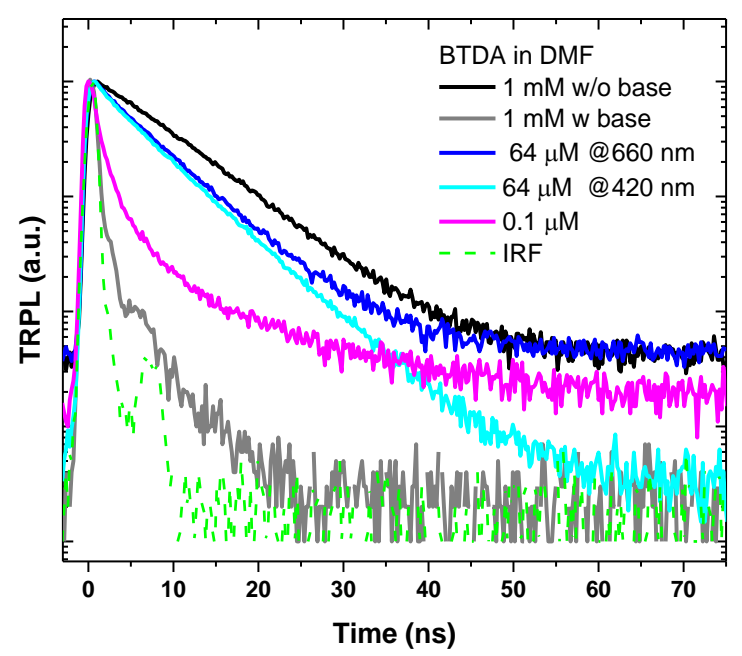

Figure 38. Normalized time-resolved emission for TBDB linker in DMF at various concentrations.

In addition, photoluminescence quantum yield measurements have been carried out for BTDB molecules at various concentrations. At the low end of the concentration 
range $(0.1 \mu \mathrm{M})$, a photoluminescence quantum yield of $1.2 \%$ is obtained. Upon increasing the concentration to 64 and $1000 \mu \mathrm{M}$, photoluminescence quantum yields of $58 \%$ and $69 \%$ are obtained, respectively, see Table 4. PLQY data suggests that monomer species have at least one dominate non-radiative decay channel, and that dimers and oligomers seem to suppress the mentioned channel. Similar to other organic molecules reported in the literature, torsional motion in the excited state can act as a non-radiative decay channel ${ }^{53,} 55,75,78$. To suppress the channel, plastic polymer matrix polymethyl methacrylate (PMMA) was used at various concentrations of BTDB. In PMMA matrix, species are embedded within the three dimensional structure of the polymer, and their torsional motion is restricted. Figure 39 shows that emission spectra and excited state lifetime measurements are identical across all concentrations, thus confirming the contribution of torsional motion to the excited state dynamics of BTDB molecules. Observing that photoluminescence quantum yield is proportional to the complexity of structure, one may suggest that structure rigidity plays a role in suppressing the nonradiative decay channel, thus increasing the photoluminescence quantum yield of the material. Moreover, upon the addition of an organic base, the photoluminescence quantum yield of the oligomer species, $1000 \mu \mathrm{M}$, dropped to $1.6 \%$, thus resembling the photoluminescence quantum yield of the monomer species at $0.1 \mu \mathrm{M}$. Therefore, PLQY measurements are in full agreement with the hydrogen-bond formation hypothesis. In chapter 1 , it is shown that photoluminescence quantum yield is proportional to excited state lifetime. Looking at data presented in this section, one finds that PLQY data is consistent with excited state lifetime measurements. 
Table 4. Fluoresence-quantum yield data and excited state lifetime measurements, along with corresponding global fitting using exponential equation: $A_{1} e^{-t / \tau 1}+A_{2} e^{-t / \tau 2}$ of $B T D B$ at various concentrations in the presence and absence pf organic base

\begin{tabular}{|c|c|c|c|c|}
\hline \multirow{2}{*}{$\begin{array}{c}\text { Concentration } \\
(\mu \mathrm{M})\end{array}$} & $\begin{array}{c}\text { Emission wavelength } \\
(\mathrm{nm})\end{array}$ & $\begin{array}{c}\text { Photoluminescence } \\
\text { Quantum Yield }(\Phi) \\
(\%)\end{array}$ & \multicolumn{2}{|c|}{$\begin{array}{c}\text { Emission lifetimes } \\
(\mathrm{ns})\end{array}$} \\
\cline { 5 - 5 } & & $\tau_{1}(\%)$ & $\tau_{2}(\%)$ \\
\hline 0.1 & --- & 1.2 & $0.95(94)$ & $8.3(6)$ \\
\hline 64 & 420 & 58 & $4.7(66)$ & $8.3(34)$ \\
\cline { 5 - 6 } & 660 & $5.3(72)$ & $8.3(28)$ \\
\hline 64 & ---- & 69 & --- & $8.3(100)$ \\
\hline 1000 & ---- & 1.6 & $0.6(99)$ & $8.3(1)$ \\
\hline 1000 with base & & & & \\
\hline
\end{tabular}
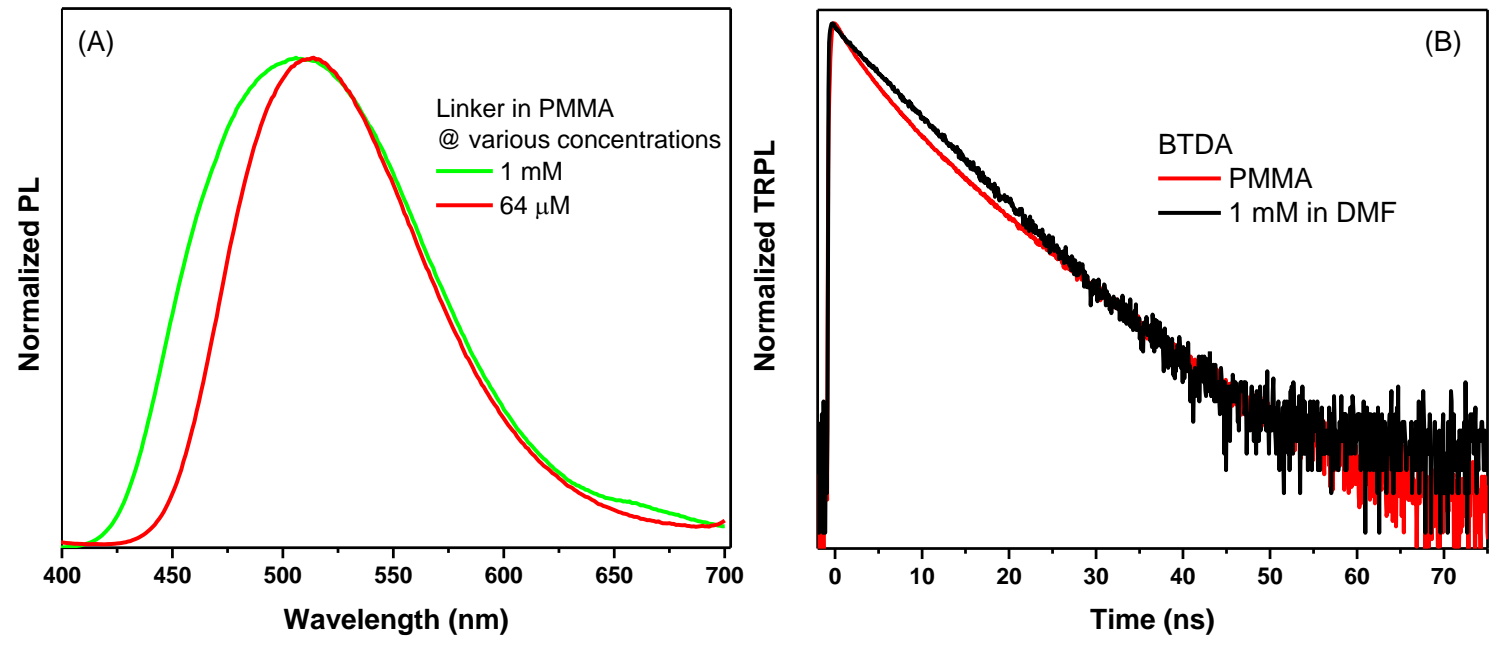

Figure 39. (A) Normalized emission spectra of BTDB linker at various concentrations in $P M M A$. (B) TRPL for linker in DMF with $1 \mathrm{mM}$ concentration and low concentration embedded in PMMA.

Figure 40 summarizes the excited state processes for BTDB molecules in all three different forms: monomer, dimer, and oligomer species. Looking at the monomer species, the molecule can be locally stabilized around a dihedral angle of $37.5^{\circ}$ in the ground state. 
Then upon excitation, the monomer emits at $480 \mathrm{~nm}$, while relaxing to the ground state through an active deactivating non-radiative channel "conical intersection". This is in full agreement with the observed short excited state lifetime, and low photoluminescence quantum yield measurement, 1.2\% QY. On the other hand, upon dimerization, the potential energy of the dimer species is changed in a way to weaken the conical intersection, as indicated by the enhanced excited state lifetime and photoluminescence quantum yield value. Dimer species can give rise in this case into two emission peaks at $420 \mathrm{~nm}$ and $620 \mathrm{~nm}$ that correspond to the two minima of the potential energy diagram in Figure 39. Similar to the dimers, oligomer species' potential energy diagram does not feature a conical intersection, thus resulting in an enhanced excited state lifetime and photoluminescence quantum yield value. Moreover, the potential energy diagram of the oligomer species features a single minimum, which stems from the single emission peak spectrum of the oligomer species at $1000 \mu \mathrm{M}$. 


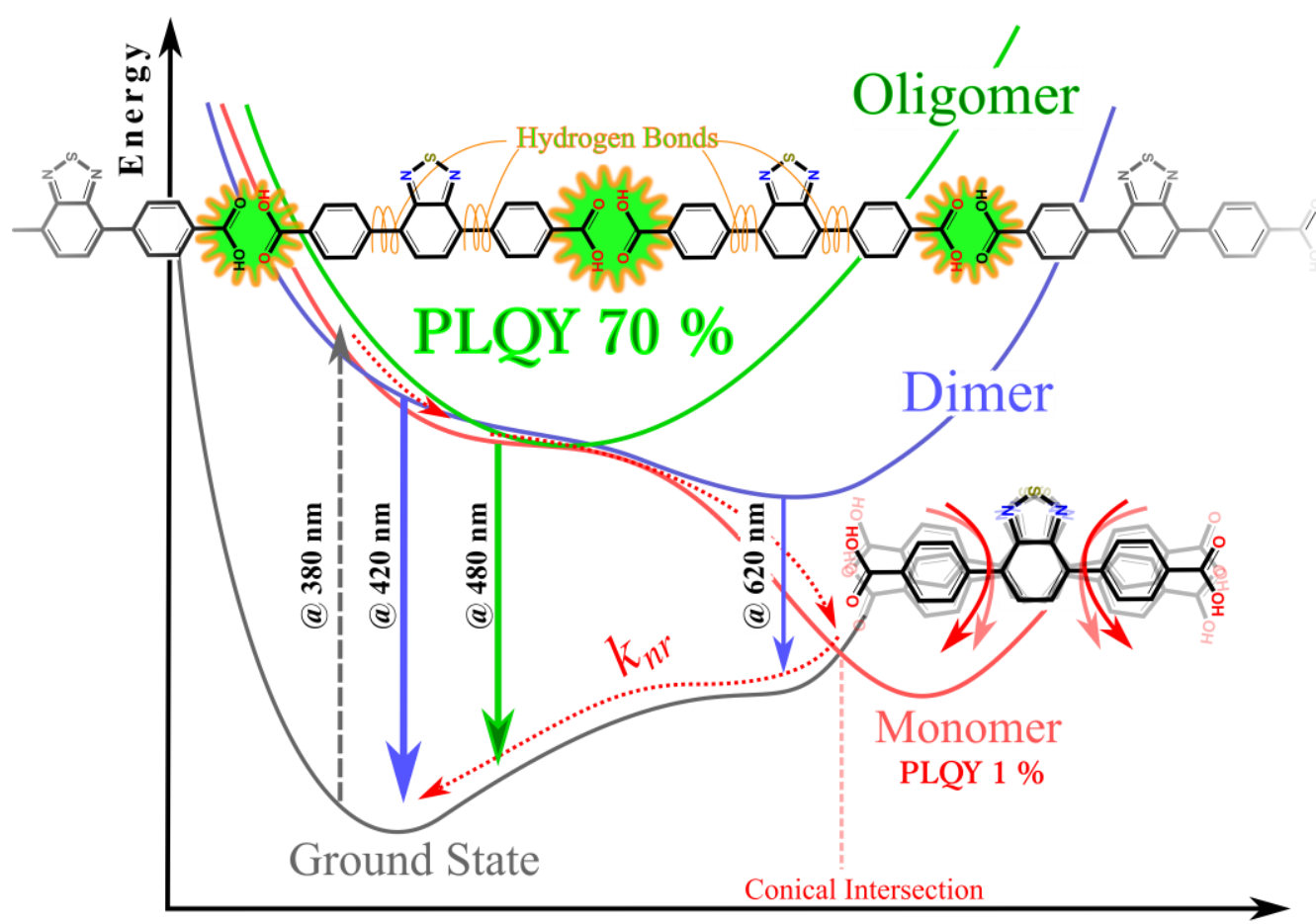

Nuclear Coordinates

Figure 40. A scheme featuring the potential energy diagrams vs. nuclear coordinates of all $B T D B$ species discussed in the thesis: monomer, dimer, and oligomer species.

\subsection{Conclusion}

In this thesis, it has been demonstrated that organic molecules with terminal carboxylic acid groups can induce dimerization and oligomerization processes at extremely low concentrations, i.e. in the nano- and micro- molar regimes, while featuring high equilibrium constants of formation. Dimer and oligomer species formations at various concentrations are heavily influenced by the hydrogen-bond formation through the terminal carboxylic acid groups, and give rise to spectral changes of the sample of interest. Experimental results and theoretical calculations verify that dimerization and oligomerization processes centered on hydrogen bond formation, and that hydrogen bond formation facilitates the formation of distinct species with different photophysical 
behavior. Moreover, photophysical properties, such as excited state lifetime and photoluminescence quantum yield measurements, are influenced by structure rigidity. This is clearly observed by how dimerization and oligomerization processes suppress torsional motion of BTDB molecules, which acts as a non-radiative decay channel. Therefore, controlling structure rigidity of a molecule can help tune photophysical properties of materials to be implemented in any application of interest. In the case of BTDB molecules, structure rigidity of oligomers facilitated the growth of the photoluminescence quantum yield of the molecule by 70 folds with respect to the single monomer species. This study hopes to spark interest in new frontiers in the field of science and engineering to develop strategies on how materials can be designed on the fundamental level to meet desired application needs.

\subsection{Future Perspective}

Metal Organic Frameworks (MOFs) have gained extensive attention in recent years due to their high porosity, along with synthesis flexibility with respect to componentchoice, size, shape, and functionality ${ }^{4}$. Looking at MOF frameworks from a spectroscopic perspective, one can come to realize that organic linkers within MOF structures tend to be rigid, and that their movements are restricted. Therefore, extending our findings from the BTDB study, it can be forecasted that incorporating photoluminescent organic linkers in a MOF framework, would help increase photoluminescence quantum yield, and elongate their excited state lifetimes. It is evident that restricting the movement of an

organic linker can potentially suppress non-radiative decay channels, such as torsional 
motion in the case of BTDB. Therefore, given the flexibility that researchers have in synthesizing MOF frameworks, several factors, such as choice of precursors, shape, are available to control the rigidity of organic linkers, thus being able tune the spectroscopic properties of interest to meet desired needs.

In addition, controlling photophysical performance of BTDB molecules stems from the presence of two carboxylic acid functional groups, the resulted long-range hydrogen bonding interactions as well as the hindrance of the torsional motions of the benzothiazole moiety. Therefore, one may suggest the incorporation of several carboxylic acid groups within an organic linker to enhance hydrogen-bond formation, thus improving the rigidity of the molecule and its photophysical properties. Organic linkers can be further functionalized with hydrogen acceptors and donors, such as alcohol and amine groups, to enhance the resulted molecular rigidity. On the other hand, one may propose the synthesis of organic linkers in which torsional motions are not geometrically possible. For example, synthesizing a highly cyclic molecule can easily forbid any torsional motions from taking place, thus improving molecular rigidity and enhancing the photophysical properties of the molecule. The mentioned ideas are merely suggestions to improve molecular rigidity, and that other ideas can be implemented as well.

Improved photophysical properties as a result of molecular rigidity lead to materials with enhanced photoluminescence quantum yields and excited state lifetimes. Such materials are highly valuable, and can be utilized in emission-based applications in the industry. The mentioned type of applications include LEDs, lasers, and display 
applications. Therefore, the proposed approach hopes to redirect material development efforts towards a goal-driven method, which lies at the main foundation of the materials by design methodology. 


\section{BIBLIOGRAPHY}

1. Stennett, E. M.; Ciuba, M. A.; Levitus, M., Photophysical processes in single molecule organic fluorescent probes. Chemical Society Reviews 2014, 43 (4), 1057-1075.

2. Molla, M. R.; Gehrig, D.; Roy, L.; Kamm, V.; Paul, A.; Laquai, F.; Ghosh, S., SelfAssembly of Carboxylic Acid Appended Naphthalene Diimide Derivatives with Tunable Luminescent Color and Electrical Conductivity. Chemistry-A European Journal 2014, 20 (3), 760-771.

3. Malakar, P.; Modak, D.; Prasad, E., Pure white light emission from organic molecules using solvent induced selective self-assembly. Chemical Communications 2016, 52 (23), 4309-4312.

4. Zhou, H.-C.; Long, J. R.; Yaghi, O. M., Introduction to metal-organic frameworks. ACS Publications: 2012.

5. Liu, Y.; Howarth, A. J.; Vermeulen, N. A.; Moon, S.-Y.; Hupp, J. T.; Farha, O. K., Catalytic degradation of chemical warfare agents and their simulants by metal-organic frameworks. Coordination Chemistry Reviews 2017, 346, 101-111.

6. Stock, N.; Biswas, S., Synthesis of metal-organic frameworks (MOFs): routes to various MOF topologies, morphologies, and composites. Chemical reviews 2011, 112 (2), 933-969.

7. Furukawa, H.; Ko, N.; Go, Y. B.; Aratani, N.; Choi, S. B.; Choi, E.; Yazaydin, A. Ö.; Snurr, R. Q.; O’Keeffe, M.; Kim, J., Ultrahigh porosity in metal-organic frameworks. Science 2010, 329 (5990), 424-428.

8. Kim, J.; Chen, B.; Reineke, T. M.; Li, H.; Eddaoudi, M.; Moler, D. B.; O'Keeffe, M.; Yaghi, O. M., Assembly of metal- organic frameworks from large organic and inorganic secondary building units: new examples and simplifying principles for complex structures. Journal of the American Chemical Society 2001, 123 (34), 8239-8247.

9. Mostakim, S.; Biswas, S., A thiadiazole-functionalized Zr (iv)-based metal-organic framework as a highly fluorescent probe for the selective detection of picric acid. CrystEngComm 2016, 18 (17), 3104-3113.

10. Allendorf, M.; Bauer, C.; Bhakta, R.; Houk, R., Luminescent metal-organic frameworks. Chemical Society Reviews 2009, 38 (5), 1330-1352.

11. Lama, P.; Aijaz, A.; Sanudo, E. C.; Bharadwaj, P. K., Synthesis, structure, and magnetic properties of cobalt (II) coordination polymers from a new tripodal 
carboxylate ligand: Weak ferromagnetism and metamagnetism. Crystal Growth \& Design 2009, 10 (1), 283-290.

12. Almansaf, A. Charge Carrier Dynamics at Silver Nanocluster-Molecular Acceptor Interfaces. 2017.

13. Ovesný, M., Computational methods in single molecule localization microscopy. 2016.

14. Salthouse, J. A.; Ware, M. J., Point group character tables and related data. CUP Archive: 1972.

15. Swinehart, D., The beer-lambert law. Journal of chemical education 1962, 39 (7), 333.

16. Demchenko, A. P.; Tomin, V. I.; Chou, P.-T., Breaking the Kasha rule for more efficient photochemistry. Chemical reviews 2017, 117 (21), 13353-13381.

17. Condon, E., The Franck-Condon principle and related topics. American journal of physics 1947, 15 (5), 365-374.

18. Chmyrov, A. Photo-induced dark states influorescence spectroscopyinvestigations \& applications. KTH, 2010.

19. Introduction to Fluorescence. In Principles of Fluorescence Spectroscopy, Lakowicz, J. R., Ed. Springer US: Boston, MA, 2006; pp 1-26.

20. Petdum, A.; Panchan, W.; Swanglap, P.; Sirirak, J.; Sooksimuang, T.; Wanichacheva, N., "Turn-ON"[5] helicene-based fluorescence sensor with very large Stokes shift for highly selective detection of Ag+ and AgNPs. Sensors and Actuators B: Chemical 2018, 259, 862-870.

21. Herman, B.; Frohlich, V. C.; Lakowicz, J.; Murphy, D.; Spring, K.; Davidson, M., Basic concepts in Fluorescence. Microscopy Resource center Olympus. http://www. olympusmicro. co/primer/techniques/fluorescence/fluorescenceintro. htm/ 2009.

22. Solvent and Environmental Effects. In Principles of Fluorescence Spectroscopy, Lakowicz, J. R., Ed. Springer US: Boston, MA, 2006; pp 1-26.

23. Albani, J. R., Principles and applications of fluorescence spectroscopy. John Wiley \& Sons: 2008.

24. Mohammed, O. F.; Kwon, O. H.; Othon, C. M.; Zewail, A. H., Charge Transfer Assisted by Collective Hydrogen-Bonding Dynamics. Angewandte Chemie International Edition 2009, 48 (34), 6251-6256. 
25. El-Ballouli, A. a. O.; Alarousu, E.; Bernardi, M.; Aly, S. M.; Lagrow, A. P.; Bakr, O. M.; Mohammed, O. F., Quantum confinement-tunable ultrafast charge transfer at the $\mathrm{PbS}$ quantum dot and phenyl-C61-butyric acid methyl ester interface. Journal of the American Chemical Society 2014, 136 (19), 6952-6959.

26. Quenching of Fluorescence. In Principles of Fluorescence Spectroscopy, Lakowicz, J. R., Ed. Springer US: Boston, MA, 2006; pp 277-330.

27. Ahmed, G. H.; Liu, J.; Parida, M. R.; Murali, B.; Bose, R.; AlYami, N. M.; Hedhili, M. N.; Peng, W.; Pan, J.; Besong, T. M., Shape-tunable charge carrier dynamics at the interfaces between perovskite nanocrystals and molecular acceptors. The journal of physical chemistry letters 2016, 7 (19), 3913-3919.

28. Singh, P. K.; Kumbhakar, M.; Pal, H.; Nath, S., Ultrafast torsional dynamics of protein binding dye thioflavin-T in nanoconfined water pool. The Journal of Physical Chemistry B 2009, 113 (25), 8532-8538.

29. Virgili, T.; Forni, A.; Cariati, E.; Pasini, D.; Botta, C., Direct evidence of torsional motion in an aggregation-induced emissive chromophore. The Journal of Physical Chemistry C 2013, 117 (51), 27161-27166.

30. Steiner, T., The hydrogen bond in the solid state. Angewandte Chemie International Edition 2002, 41 (1), 48-76.

31. Kerszulis, J. A., Cary UV/Vis/NIR 500 User Guide and Tutorial for Using the Cray 500 in the Reynolds Research group. 2012.

32. Brandt, M., Spectrofluorometers and Fluorescence Phenomena. 1999.

33. Applications and Use of Integrating Spheres. PerkinElmer Life and Analytical Sciences 2004.

34. El-Zohry, A. M. Exploring Organic Dyes for Grätzel Cells Using Time-Resolved Spectroscopy. Acta Universitatis Upsaliensis, 2015.

35. Becker, E. D., High resolution NMR: theory and chemical applications. Elsevier: 1999.

36. Jacobsen, N. E., NMR spectroscopy explained: simplified theory, applications and examples for organic chemistry and structural biology. John Wiley \& Sons: 2007.

37. Atkins, P.; Jones, L., Chemical principles: The quest for insight. Macmillan: 2007.

38. Gross, E. K.; Dreizler, R. M., Density functional theory. Springer Science \& Business Media: 2013; Vol. 337. 
39. Adamo, C.; Jacquemin, D., The calculations of excited-state properties with TimeDependent Density Functional Theory. Chemical Society Reviews 2013, 42 (3), 845-856.

40. Gross, E.; Kohn, W., Time-dependent density-functional theory. In Advances in quantum chemistry, Elsevier: 1990; Vol. 21, pp 255-291.

41. Kaur, N.; Singh, M.; Pathak, D.; Wagner, T.; Nunzi, J. M., Organic materials for photovoltaic applications: Review and mechanism. Synthetic Metals 2014, 190, 20-26.

42. Qian, H.; Cousins, M. E.; Horak, E. H.; Wakefield, A.; Liptak, M. D.; Aprahamian, I., Suppression of Kasha's rule as a mechanism for fluorescent molecular rotors and aggregation-induced emission. Nature Chemistry 2016, 9, 83.

43. Stennett, E. M. S.; Ciuba, M. A.; Levitus, M., Photophysical processes in single molecule organic fluorescent probes. Chemical Society Reviews 2014, 43 (4), 1057-1075.

44. Wurthner, F.; Kaiser, T. E.; Saha-Moller, C. R., J-Aggregates: From Serendipitous Discovery to Supramolecular Engineering of Functional Dye Materials. Angew Chem Int Edit 2011, 50 (15), 3376-3410.

45. Sabate, R.; Estelrich, J., Determination of the dimerization constant of pinacyanol: Role of the thermochromic effect. Spectrochim Acta A 2008, 70 (2), 471-476.

46. Qin, A.; Jim, C. K.; Tang, Y.; Lam, J. W.; Liu, J.; Mahtab, F.; Gao, P.; Tang, B. Z., Aggregation-enhanced emissions of intramolecular excimers in disubstituted polyacetylenes. Journal of Physical Chemistry B 2008, 112 (31), 9281-9288.

47. Luo, J.; Xie, Z.; Lam, J. W.; Cheng, L.; Chen, H.; Qiu, C.; Kwok, H. S.; Zhan, X.; Liu, Y.; Zhu, D., Aggregation-induced emission of 1-methyl-1, 2, 3, 4, 5-pentaphenylsilole. Chemical Communications 2001, (18), 1740-1741.

48. Gilani, A. G.; Moghadam, M.; Hosseini, S. E.; Zakerhamidi, M. S., A comparative study on the aggregate formation of two oxazine dyes in aqueous and aqueous urea solutions. Spectrochim Acta A 2011, 83 (1), 100-105.

49. Arbeloa, F. L.; Ojeda, P. R.; Arbeloa, I. L., Dimerization and Trimerization of Rhodamine 6g in Aqueous-Solution - Effect on the Fluorescence Quantum Yield. J Chem Soc Farad T 2 1988, 84, 1903-1912.

50. Satchell, D. P.; Wardell, J. L., Dimerization of Carboxylic Acids in ODichlorobenzene. T Faraday Soc 1965, 61 (510P), 1199-\&.

51. Zhang, W. Q.; Li, Q. Y.; Cheng, J. Y.; Cheng, K.; Yang, X. Y.; Li, Y. W.; Zhao, X. S.; Wang, X. J., Ratiometric Luminescent Detection of Organic Amines Due to the Induced 
Lactam-Lactim Tautomerization of Organic Linker in a Metal-Organic Framework. Acs Appl Mater Inter 2017, 9 (37), 31352-31356.

52. Usman, A.; Mohammed, O. F.; Nibbering, E. T. J.; Dong, J.; Solntsev, K. M.; Tolbert, L. M., Excited-state structure determination of the green fluorescent protein chromophore. J. Am. Chem. Soc. 2005, 127 (32), 11214-11215.

53. Zietz, B.; Gabrielsson, E.; Johansson, V.; El-Zohry, A. M.; Sun, L.; Kloo, L., Photoisomerization of The Cyanoacrylic Acid Acceptor Group-A Potential Problem for Organic Dyes in Solar Cells. Physical Chemistry Chemical Physics 2014, 16 (6), 2251-2255.

54. Rosspeintner, A.; Lang, B.; Vauthey, E., Ultrafast Photochemistry in Liquids. Annu. Rev. Phys. Chem. 2013, 64, 247-271.

55. El-Zohry, A.; Orthaber, A.; Zietz, B., Isomerization and Aggregation of the Solar Cell Dye D149. Journal of Physical Chemistry C 2012, 116 (50), 26144-26153.

56. Lin, Y. D.; Chow, T. J., Geometrical Effect of Stilbene on The Performance of Organic Dye-Sensitized Solar Cells. J Mater Chem 2011, 21 (38), 14907-14916.

57. Polli, D.; Altoe, P.; Weingart, O.; Spillane, K. M.; Manzoni, C.; Brida, D.; Tomasello, G.; Orlandi, G.; Kukura, P.; Mathies, R. A.; Garavelli, M.; Cerullo, G., Conical intersection dynamics of the primary photoisomerization event in vision. Nature 2010, 467 (7314), 440-443.

58. Levine, B. G.; Martinez, T. J., Isomerization through conical intersections. Annual Review of Physical Chemistry 2007, 58, 613-634.

59. Zietz, B.; Blomgren, F., Conical intersection in a bilirubin model - A possible pathway for phototherapy of neonatal jaundice. Chem. Phys. Lett. 2006, 420 (4-6), 556561.

60. Zietz, B.; Macphersona, A. N.; Gillbro, T., Resolution of ultrafast excited state kinetics of bilirubin in chloroform and bound to human serum albumin. Phys. Chem. Chem. Phys. 2004, 6 (19), 4535-4537.

61. Mostakim, S. K.; Biswas, S., A thiadiazole-functionalized Zr(IV)-based metalorganic framework as a highly fluorescent probe for the selective detection of picric acid. Crystengcomm 2016, 18 (17), 3104-3113.

62. Neto, B. A. D.; Carvalho, P. H. P. R.; Correa, J. R., Benzothiadiazole Derivatives as Fluorescence Imaging Probes: Beyond Classical Scaffolds. Accounts Chem Res 2015, 48 (6), 1560-1569. 
63. Hong, Y. N.; Lam, J. W. Y.; Tang, B. Z., Aggregation-induced emission. Chem. Soc. Rev. 2011, 40 (11), 5361-5388.

64. Mei, J.; Leung, N. L. C.; Kwok, R. T. K.; Lam, J. W. Y.; Tang, B. Z., AggregationInduced Emission: Together We Shine, United We Soar! Chem. Rev. 2015, 115 (21), 11718-11940.

65. Debnath, T.; Maity, P.; Lobo, H.; Singh, B.; Shankarling, G. S.; Ghosh, H. N., Extensive Reduction in Back Electron Transfer in Twisted Intramolecular Charge-Transfer (TICT) Coumarin-Dye-Sensitized TiO2 Nanoparticles/Film: A Femtosecond Transient Absorption Study. Chem. Eur. J. 2014, 20 (12), 3510-3519.

66. Yushchenko, O.; Villamaina, D.; Sakai, N.; Matile, S.; Vauthey, E., Comparison of Charge-Transfer Dynamics of Naphthalenediimide Triads in Solution and $\pi$-Stack Architectures on Solid Surfaces. J. Phys. Chem. C 2015, 119 (27), 14999-15008.

67. Lakowicz, J. R., Principles of fluorescence spectroscopy. Springer: 2007.

68. El-Zohry, A. M.; Zietz, B., Concentration and Solvent Effects on the Excited State Dynamics of the Solar Cell Dye D149: The Special Role of Protons. Journal of Physical Chemistry C 2013, 117 (13), 6544-6553.

69. El-Zohry, A. M., The origin of slow electron injection rates for indoline dyes used in dye-sensitized solar cells. Dyes and Pigments 2019, 160, 671-674.

70. El-Zohry, A. M.; Karlsson, M., Gigantic Relevance of Twisted Intramolecular Charge Transfer for Organic Dyes Used in Solar Cells. J. Phys. Chem. C 2018.

71. Hussain, M.; El-Zohry, A. M.; Gobeze, H. B.; Zhao, J. Z.; D'Souza, F.; Mohammed, O. F., Intramolecular Energy and Electron Transfers in Bodipy Naphthalenediimide Triads. J. Phys. Chem. A 2018, 122 (29), 6081-6088.

72. Dereka, B.; Koch, M.; Vauthey, E., Looking at Photoinduced Charge Transfer Processes in the IR: Answers to Several Long-Standing Questions. Acc. Chem. Res. 2017, $50(2), 426-434$.

73. Zhong, D. P., Electron Transfer Mechanisms of DNA Repair by Photolyase. Annu. Rev. Phys. Chem. 2015, 66, 691-715.

74. Lide, D. R., Handbook of organic solvents. CRC Press: 1994.

75. El-Zohry, A. M.; Roca-Sanjuan, D.; Zietz, B., Ultrafast Twisting of the Indoline Donor Unit Utilized in Solar Cell Dyes: Experimental and Theoretical Studies. Journal of Physical Chemistry C 2015, 119 (5), 2249-2259. 
76. Rosspeintner, A.; Lang, B.; Vauthey, E., Ultrafast Photochemistry in Liquids. Annu Rev Phys Chem 2013, 64, 247-271.

77. Brown, W. H.; Poon, T.; Poon, T., Introduction to organic chemistry. John Wiley \& Sons: 2014.

78. El-Zohry, A. M.; Cong, J.; Karlsson, M.; Kloo, L.; Zietz, B., Ferrocene as a rapid charge regenerator in dye-sensitized solar cells. Dyes and Pigments 2016, 132, 360-368. 